\title{
Copper-Catalyzed Alkene Aziridination with N-Tosyloxycarbamates
}

Hélène Lebel,* Sylvain Lectard and Michaël Parmentier

Département de chimie, Université de Montréal, Montréal, Québec, Canada, H3C 3J7

Supporting Information

\section{Table of Contents}

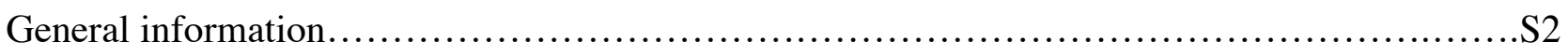

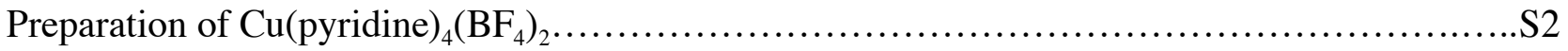

General Procedures................................................................. S3

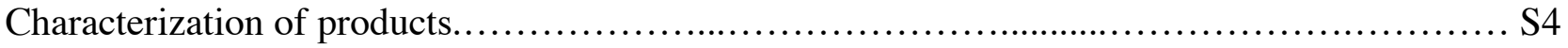

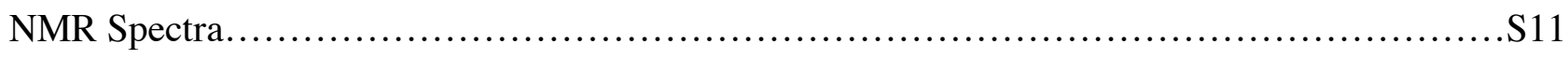


General: The commercially available alcohols were purified using standard methods prior to use. $\mathrm{K}_{2} \mathrm{CO}_{3}$ was purchased from Aldrich and finely powdered before use. $\mathrm{Cu}\left(\mathrm{BF}_{4}\right)_{2}$ was obtained from $\mathrm{Cu}\left(\mathrm{BF}_{4}\right)_{2} \cdot \mathrm{xH}_{2} \mathrm{O}$ after drying under high vacuum for one week. Known copper complexes were prepared according to literature procedures. ${ }^{1}$ Known allylic $N$-hydroxycarbamates and allylic $N$ tosyloxycarbamates were prepared according to literature procedure. ${ }^{2}$ 2,2,2-trichloroethyl- $N$ tosyloxycarbamate $\mathbf{1 0}$ was prepared according to a published procedure. ${ }^{3}$ Analytical thin layer chromatography (TLC) was performed using EM Reagent $0.25 \mathrm{~mm}$ silica gel $60-\mathrm{F}$ plates. Visualization of the developed chromatogram was performed by UV absorbance, aqueous cerium molybdate, ethanolic phosphomolybdic acid, or aqueous potassium permanganate. Flash chromatography was performed using EM Silica Gel 60 (230-400 mesh) with the indicated solvent system. Optical rotations were measured on a Perkin-Elmer 341 digital polarimeter at $589 \mathrm{~nm}$. Data are reported as follows: $[\alpha]_{\mathrm{D}}$ temp, concentration $(c \mathrm{~g} / 100 \mathrm{~mL})$, and solvent. Infrared spectra were recorded on a Perkin Elmer Spectrum One FTIR spectrometer equipped with a Golden Gate Diamond ATR and are reported in reciprocal centimeters (cm-1). Only the most important and relevant frequencies are reported. ${ }^{1} \mathrm{H}$ NMR spectra were recorded in $\mathrm{CDCl}_{3}$, unless otherwise noted, on a Bruker AV-400, a Bruker ARX-400, a Bruker AMX-300 or a Bruker AV-300 spectrometers (400, 400, 300 and $300 \mathrm{MHz}$ respectively). Chemical shifts are reported in ppm on the $\delta$ scale from an internal standard of residual chloroform (7.26 ppm). Data are reported as follows: chemical shift, multiplicity $(\mathrm{s}=$ singlet, $\mathrm{d}=$ doublet, $\mathrm{t}=$ triplet, $\mathrm{q}=$ quartet, $\mathrm{qn}=$ quintet, $\mathrm{m}=$ multiplet and $\mathrm{br}=$ broad), coupling constant in $\mathrm{Hz}$, integration. ${ }^{13} \mathrm{C}$ NMR spectra were recorded in $\mathrm{CDCl}_{3}$, unless otherwise noted, on a Bruker AV-400, a Bruker ARX-400, a Bruker AMX-300 or a Bruker AV-300 spectrometers (100, 100, 75 and 75 MHz respectively) with complete proton decoupling. Chemical shifts are reported in ppm from the central peak of $\mathrm{CDCl}_{3}(76.9 \mathrm{ppm})$ on the $\delta$ scale. Mass spectra were obtained on a LC-MSD TOF (ESI) Agilent Technologies high resolution from the Centre régional de spectrométrie de masse de l'Université de Montréal.

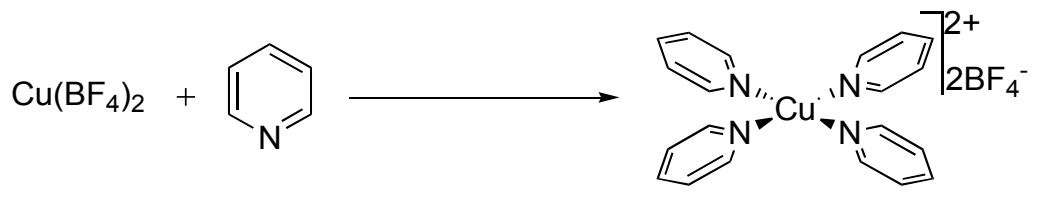

\section{Tetrakis(pyridine) copper (II) bis(tetrafluoroborate)}

To a suspension of copper tetrafluoroborate (II) $(1.19 \mathrm{~g}, 5.00 \mathrm{mmol})$ in benzene $(50 \mathrm{~mL})$ was added distilled pyridine $(8.10 \mathrm{~mL}, 100 \mathrm{mmol})$. The heterogeneous solution was heated to $80{ }^{\circ} \mathrm{C}$ for $15 \mathrm{~min}$ then cooled to room temperature. The purple suspension was stirred for 24 hours. Ether $(50 \mathrm{~mL}) \mathrm{was}$ added and the blue solid was then filtered, washed with ether and dried under reduced pressure, affording the desired complex $(2.67 \mathrm{~g}, 97 \%)$ as a light purple solid. Monocrystals were obtained by diffusing dichloromethane into acetonitrile and were resolved by X-ray crystal structure analysis

\footnotetext{
1 (a) Haynes, J. S.; Rettig, S. J.; Sams, J. R.; Trotter, J.; Thompson, R. C. Inorg. Chem. 1988, 27, 1237-1241. (b) Mohr, F.; Binfield, S. A.; Fettinger, J. C.; Vedernikov, A. N. J. Org. Chem. 2005, 70, 4833-4839.

2 Lebel, H.; Huard, K.; Lectard, S. J. Am. Chem. Soc. 2005, 127, 14198-14199.

3 Lebel, H.; Huard, K. Org. Lett. 2007, 9, 639-642.
} 


\section{Procedure A: Preparation of allylic $N$-hydroxycarbamates ${ }^{4}$}

The unsaturated alcohol $(25.0 \mathrm{mmol})$ was dissolved at room temperature in DCM $(50 \mathrm{ml})$. Carbonyl diimidazole $(4.46 \mathrm{~g}, 27.5 \mathrm{mmol})$ was added in one portion and the solution was stirred for $1 \mathrm{~h}$. The solution was washed with a saturated solution of $\mathrm{NH}_{4} \mathrm{Cl}(3 \times 50 \mathrm{ml})$. The organic layer was dried over $\mathrm{MgSO}_{4}$, filtered and concentrated to give an oil. The previous oil was dissolved in pyridine $(85 \mathrm{ml})$ at room temperature and $\mathrm{NH}_{2} \mathrm{OH} \cdot \mathrm{HCl}(5.21 \mathrm{~g}, 75.0 \mathrm{mmol})$ was added in one portion. The solution was stirred for $3 \mathrm{~h}$. DCM $(100 \mathrm{ml})$ was added followed by a $10 \%$ solution of sulphuric acid $(125 \mathrm{ml})$. The two layers were separated and the organic layer was washed with $10 \%$ solution of sulphuric acid $(3 \mathrm{x}$ $150 \mathrm{~mL}$ ), then dried over $\mathrm{MgSO}_{4}$, filtered and concentrated. The product was purified by flash chromatography on silica gel using AcOEt/hexanes as eluent or recrystallized from chloroform/hexanes.

\section{Procedure B: Preparation of allylic $N$-hydroxycarbamates ${ }^{5}$}

The unsaturated alcohol $(25.0 \mathrm{mmol})$ was dissolved at room temperature in $\mathrm{CH}_{3} \mathrm{CN}(150 \mathrm{ml})$. Carbonyl diimidazole $(4.86 \mathrm{~g}, 30.0 \mathrm{mmol})$ was added in one portion and the solution was stirred for $90 \mathrm{~min}$. $\mathrm{NH}_{2} \mathrm{OH} \cdot \mathrm{HCl}(8.67 \mathrm{~g}, 125 \mathrm{mmol})$ was added in one portion followed by imidazole $(6.81 \mathrm{~g}, 100 \mathrm{mmol})$. The solution was stirred for $3 \mathrm{~h}$ and a $10 \%$ solution of sulphuric acid was added until $\mathrm{pH} 2-3$. A biphasic colorless solution was obtained. The organic layer was removed and the aqueous layer was extracted with ether $(4 \times 100 \mathrm{~mL})$ and the organic layers were dried over $\mathrm{MgSO}_{4}$, filtered and concentrated. The product was purified by flash chromatography on silica gel using AcOEt/hexanes as eluent or recrystallized from chloroform/hexanes.

\section{Procedure C: Preparation of allylic $\mathrm{N}$-tosyloxycarbamates}

The $N$-hydroxycarbamate $(25.0 \mathrm{mmol})$ was dissolved in $\mathrm{Et}_{2} \mathrm{O}(50 \mathrm{~mL}) . p$-Toluenesulfonyl chloride $(4.76 \mathrm{~g}, 25.0 \mathrm{mmol})$ was added in one portion at $0{ }^{\circ} \mathrm{C}$. The solution was stirred for $15 \mathrm{~min}$ then a solution of $\mathrm{Et}_{3} \mathrm{~N}(3.20 \mathrm{ml}, 22.5 \mathrm{mmol})$ in $\mathrm{Et}_{2} \mathrm{O}(5 \mathrm{~mL})$ was slowly added at such a rate that the solution remained acidic at all time. A white precipitate rapidly appeared. The solution was filtrated and the remaining solid washed with ether. The combined filtrate was concentrated under reduced pressure. The product was purified by flash chromatography on silica gel using AcOEt/hexanes as eluent or recrystallized from chloroform/hexanes.

\section{Procedure D: Catalytic intramolecular aziridination}

The $N$-tosyloxycarbamate $(1.00 \mathrm{mmol}), \mathrm{K}_{2} \mathrm{CO}_{3}(692 \mathrm{mg}, 5.00 \mathrm{mmol})$, and $\mathrm{Cu}$ (pyridine $)_{4}(\mathrm{OTf})_{2}(14 \mathrm{mg}$, $0.020 \mathrm{mmol}$,) were dissolved in acetone $(20 \mathrm{~mL})$ at $25^{\circ} \mathrm{C}$. The mixture was vigorously stirred for $16 \mathrm{~h}$ after which time DCM was added. The solution was filtered over celite ${ }^{\circledR}$ to remove the potassium tosylate salt. The filtrate was concentrated and the residue was subjected to flash chromatography on silica gel using AcOEt/hexanes as eluent.

\section{Procedure E: Catalytic intermolecular aziridination}

$\mathrm{Cu}$ (pyridine $)_{4}\left(\mathrm{BF}_{4}\right)_{2}(28 \mathrm{mg}, 0.050 \mathrm{mmol})$ was suspended in benzene $(5 \mathrm{~mL})$ in a $20 \mathrm{~mL}$ scintillation vial under air. $\mathrm{K}_{2} \mathrm{CO}_{3}(692 \mathrm{mg}, 5.00 \mathrm{mmol})$ and the olefin $(2.50 \mathrm{mmol})$ were successively added. The heterogeneous solution was stirred for $15 \mathrm{~min}$ at $25^{\circ} \mathrm{C}$ and $10(182 \mathrm{mg}, 0.50 \mathrm{mmol})$ was added in one portion. The solution was stirred at $25{ }^{\circ} \mathrm{C}$ overnight. Ether $(15 \mathrm{~mL})$ was added and the resulting mixture was filtered. The solid was washed 4 times with $10 \mathrm{~mL}$ of ether. The combined filtrate was concentrated under reduced pressure. The residue is purified by flash chromatography on silica gel using Ether/hexanes as eluent. The silica gel was pre-treated with $1 \% \mathrm{Et}_{3} \mathrm{~N} /$ hexanes.

\footnotetext{
${ }^{4}$ Sparks, S. M.; Vargas, J. D.; Shea, K. J. Org. Lett. 2000, 2, 1473-1475.

5 Donohoe, T. J.; Chughtai, M. J.; Klauber, D. J.; Griffin, D.; Campbell, A. D. J. Am. Chem. Soc. 2006, 128, $2514-2515$.
} 


\section{Characterization of allylic $N$-hydroxycarbamates}

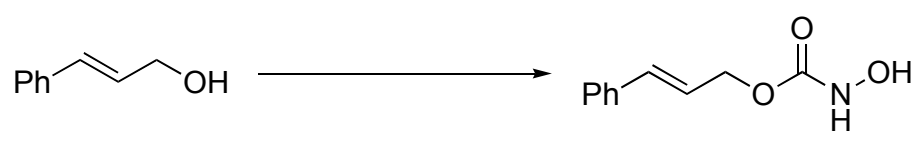

\section{(E)-3-Phenylprop-2-ene N-hydroxycarbamate}

The title compound was prepared from $(E)$-cinnamyl alcohol $(6.71 \mathrm{~g}, 50.0 \mathrm{mmol})$ according to the general procedure A. The desired $N$-hydroxycarbamate $(6.86 \mathrm{~g}, 71 \%)$ was obtained as a white solid after recrystallization. $\mathrm{R}_{f} 0.22$ (40\% AcOEt/hexanes); mp 99-100 ${ }^{\circ} \mathrm{C} .{ }^{1} \mathrm{H}$ NMR $\left(400 \mathrm{MHz}, \mathrm{CDCl}_{3}\right) \delta$ $7.42(\mathrm{~s}, 1 \mathrm{H}), 7.39-7.29(\mathrm{~m}, 5 \mathrm{H}), 7.28(\mathrm{~s}(\mathrm{br}), 1 \mathrm{H}), 6.65(\mathrm{~d}, J=16 \mathrm{~Hz}, 1 \mathrm{H}), 6.26(\mathrm{dt}, J=16,7 \mathrm{~Hz}, 1 \mathrm{H})$ $4.80(\mathrm{dd}, J=7,1 \mathrm{~Hz}, 2 \mathrm{H}) ;{ }^{13} \mathrm{C} \mathrm{NMR}\left(100 \mathrm{MHz}, \mathrm{CDCl}_{3}\right) \delta 159.1,135.8,134.7,128.5,128.1,126.6$, 122.4, 66.7; IR (neat) 3317, 3251, 2897, 1698, 1510, 1450, 1304, 1273, 1121, 972, $767.693 \mathrm{~cm}^{-1}$; HMRS (ESI) calc. for $\mathrm{C}_{10} \mathrm{H}_{11} \mathrm{NO}_{3} \mathrm{Na}[\mathrm{M}+\mathrm{Na}]^{+}$: 216.06311 ; found: 216.06279 .

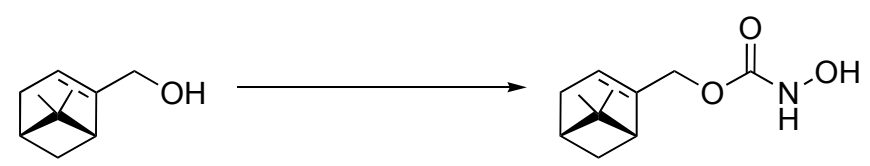

\section{2-Methyl-6,6-dimethyl-bicyclo[3.1.1]hept-2-ene $N$-hydroxycarbamate}

The title compound was prepared from (-)-myrtenol $(3.81 \mathrm{~g}, 25.0 \mathrm{mmol})$ according to the general procedure A. The desired $N$-hydroxycarbamate $(5.20 \mathrm{~g}, 98 \%)$ was obtained as a white solid after flash chromatography (30\% AcOEt/hexanes - 55\% AcOEt/hexanes). $\mathrm{R}_{f} 0.24$ (35\% AcOEt/hexanes); mp 48$50{ }^{\circ} \mathrm{C} ;[\alpha]_{\mathrm{D}}{ }^{25}=-35.3(\mathrm{c} 1.59, \mathrm{DCM}) ;{ }^{1} \mathrm{H} \mathrm{NMR}\left(400 \mathrm{MHz}, \mathrm{CDCl}_{3}\right) \delta 7.46$ (s (br), 1H), 7.34 (s (br), 1H), $5.58(\mathrm{~s}, 1 \mathrm{H}), 4.58-4.46(\mathrm{~m}, 2 \mathrm{H}), 2.41-2.37(\mathrm{~m}, 1 \mathrm{H}), 2.36-2.25(\mathrm{~m}, 2 \mathrm{H}), 2.12-2.10(\mathrm{~m}, 2 \mathrm{H}), 1.27(\mathrm{~s}, 3 \mathrm{H})$, $1.15(\mathrm{~d}, J=9 \mathrm{~Hz}, 1 \mathrm{H}), 0.80(\mathrm{~s}, 3 \mathrm{H}) ;{ }^{13} \mathrm{C} \mathrm{NMR}\left(100 \mathrm{MHz}, \mathrm{CDCl}_{3}\right) \delta 159.3,142.2,121.9,68.4,43.0$, 40.2, 37.7, 31.1, 30.9, 25.7, 20.7; IR (neat) 3284 (br), 2985, 2913, 2831, 1714, 1468, 1265, 1111, 770 $\mathrm{cm}^{-1}$; HMRS (ESI) calc. for $\mathrm{C}_{11} \mathrm{H}_{17} \mathrm{NO}_{3} \mathrm{Na}[\mathrm{M}+\mathrm{Na}]^{+}$: 234.11006; found: 234.10972.

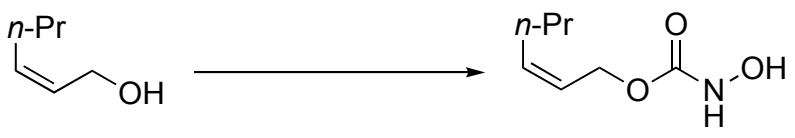

\section{(Z)-Hex-2-ene $N$-hydroxycarbamate}

The title compound was prepared from $(E)$-hex-2-en-1-ol $(2.00 \mathrm{~g}, 20.0 \mathrm{mmol})$ according to the general procedure B. The desired $N$-hydroxycarbamate $(2.79 \mathrm{~g}, 88 \%)$ was obtained as a pale yellow oil after flash chromatography (30\% AcOEt/hexanes-55\% AcOEt/hexanes). $\mathrm{R}_{f} 0.29$ (40\% AcOEt/hexanes); ${ }^{1} \mathrm{H}$ NMR (400 MHz, $\left.\mathrm{CDCl}_{3}\right) \delta 7.52(\mathrm{~s}(\mathrm{br}), 2 \mathrm{H}), 5.64(\mathrm{dt}, J=11,7 \mathrm{~Hz}, 1 \mathrm{H}), 5.52(\mathrm{dt}, J=11,7 \mathrm{~Hz}, 1 \mathrm{H})$, $4.68(\mathrm{~d}, J=7 \mathrm{~Hz}, 2 \mathrm{H}), 2.06(\mathrm{q}, J=7 \mathrm{~Hz}, 2 \mathrm{H}), 1.38$ (sextuplet, $J=7 \mathrm{~Hz}, 2 \mathrm{H}), 0.89(\mathrm{t}, J=7 \mathrm{~Hz}, 3 \mathrm{H}) ;{ }^{13} \mathrm{C}$ NMR (100 MHz, $\left.\mathrm{CDCl}_{3}\right) \delta 159.4,135.7,122.8,61.9,29.3,22.4,13.5$; IR (neat) 3294 (br), 2959, 2931, 2872, 1711, 1463, 1261, 1112, $1034 \mathrm{~cm}^{-1}$; HMRS (ESI) calc. for $\mathrm{C}_{7} \mathrm{H}_{13} \mathrm{NO}_{3} \mathrm{Na}[\mathrm{M}+\mathrm{Na}]^{+}: 182.07876$; found: 182.07844 .

\section{Characterization of $N$-tosyloxycarbamates}

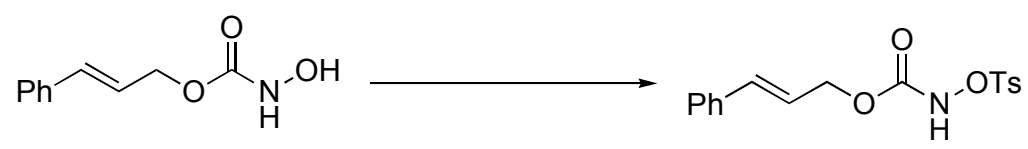

\section{(E)-3-Phenylprop-2-ene $N$ - tosyloxycarbamate}

The title compound was prepared from $(E)$-3-phenylprop-2-ene $N$-hydroxycarbamate (3.00 g, 15.5 mmol) according to the general procedure C. The desired $N$-tosyloxycarbamate $(4.86 \mathrm{~g}, 90 \%)$ was obtained as a white solid after flash chromatography (10\% AcOEt/hexanes-50\% AcOEt/hexane). $\mathrm{R}_{f}$ 
0.53 (35\% AcOEt/hexanes); mp 120-121 ${ }^{\circ} \mathrm{C} ;{ }^{1} \mathrm{H}$ NMR (400 MHz, $\left.\mathrm{CDCl}_{3}\right) \delta 7.87$ (d, J=8 Hz, 2H), 7.75 (s (br), 1H), 7.36-7.27 (m, 7H), 6.55 (d, $J=16 \mathrm{~Hz}, 1 \mathrm{H}), 6.07$ (dt, $J=16,7 \mathrm{~Hz}, 1 \mathrm{H}), 4.66$ (dd, $J=$ 7, $1 \mathrm{~Hz}, 2 \mathrm{H}), 2.36(\mathrm{~s}, 3 \mathrm{H}) ;{ }^{13} \mathrm{C} \mathrm{NMR}\left(100 \mathrm{MHz}, \mathrm{CDCl}_{3}\right) \delta 155.1,146.0,135.6,135.1,130.0,129.6$, 129.4, 128.5, 128.3, 126.5, 121.4, 67.3, 21.6; IR (neat) 3173, 2948, 1762, 1732, 1595, 1489, 1364, 1252, 1197, 1181, 963, 733, $689 \mathrm{~cm}^{-1}$; HMRS (ESI) calc. for $\mathrm{C}_{17} \mathrm{H}_{17} \mathrm{NO}_{5} \mathrm{~S}[\mathrm{M}+\mathrm{Na}]^{+}: 370.07196$; found: 370.07292 .<smiles>CC1(COC(=O)NOCCC2CC3CCC2C3)CC2CC1CC2COC(=O)NO</smiles>

\section{2-Methyl-6,6-dimethyl-bicyclo[3.1.1]hept-2-ene $N$-tosyloxycarbamate}

The title compound was prepared from 2-methyl-6,6-dimethyl-bicyclo[3.1.1]hept-2-ene $N$-hydroxycarbamate $(1.00 \mathrm{~g}, 4.7 \mathrm{mmol})$ according to the general procedure C. The desired $N$-tosyloxycarbamate $(1.27 \mathrm{~g}, 73 \%)$ was obtained as an oil after flash chromatography $(10 \%$ AcOEt/hexanes$50 \%$ AcOEt/hexanes). $\mathrm{R}_{f} 0.26(\mathrm{DCM}) ;[\mathrm{a}]_{\mathrm{D}}{ }^{25}=-9.59(c 0.98, \mathrm{DCM}) ;{ }^{1} \mathrm{H} \mathrm{NMR}\left(300 \mathrm{MHz}, \mathrm{CDCl}_{3}\right) \delta$ 7.88-7.85 (m, 3H), $7.36(\mathrm{~d}, J=8 \mathrm{~Hz}, 2 \mathrm{H}), 5.47$ (s (br), 1H), 4.40-4.36 (m, 2H), 2.46 (s, 3H), 2.41-2.23 $(\mathrm{m}, 3 \mathrm{H}), 2.12-2.04(\mathrm{~m}, 1 \mathrm{H}), 1.99(\mathrm{dt}, J=6,1 \mathrm{~Hz}, 1 \mathrm{H}), 1.26(\mathrm{~s}, 3 \mathrm{H}), 1.10(\mathrm{~d}, J=9 \mathrm{~Hz}, 1 \mathrm{H}), 0.75(\mathrm{~s}$, $3 \mathrm{H}) ;{ }^{13} \mathrm{C} \mathrm{NMR}\left(75 \mathrm{MHz}, \mathrm{CDCl}_{3}\right) \delta 155.3,145.9,141.7,130.2,129.6,129.4,122.7,69.4,43.1,40.4$, 37.9, 31.3, 31.1, 25.9, 21.7, 20.8; IR (neat) 3276 (br), 2919, 1744, 1597, 1456, 1386, 1232, 1192, 1179, 1091, 813, 738, $661 \mathrm{~cm}^{-1}$; HMRS (ESI) calc. for $\mathrm{C}_{18} \mathrm{H}_{23} \mathrm{NO}_{5} \mathrm{SNa}[\mathrm{M}+\mathrm{Na}]^{+}:$388.1189; found: 388.1192 .

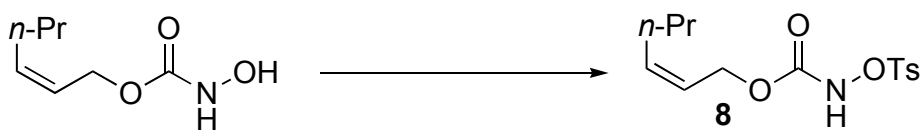

\section{(Z)-Hex-2-ene $N$-tosyloxycarbamate (8)}

The title compound was prepared from $(Z)$-hex-2-ene $N$-hydroxycarbamate $(2.79 \mathrm{~g}, 17.5 \mathrm{mmol})$ according to the general procedure $\mathbf{C}$. The desired $N$-tosyloxycarbamate $(3.53 \mathrm{~g}, 64 \%)$ was obtained as a colorless oil after flash chromatography (10\% AcOEt/hexanes-50\% AcOEt/hexanes). $\mathrm{R}_{f} 0.54(35 \%$ AcOEt/hexanes); ${ }^{1} \mathrm{H}$ NMR $\left(400 \mathrm{MHz}, \mathrm{CDCl}_{3}\right) \delta 8.33(\mathrm{~s}, 1 \mathrm{H}), 7.84(\mathrm{~d}, J=8 \mathrm{~Hz}, 2 \mathrm{H}), 7.33(\mathrm{~d}, J=8 \mathrm{~Hz}$, $2 \mathrm{H}), 5.63-5.54(\mathrm{~m}, 1 \mathrm{H}), 5.34-5.24(\mathrm{~m}, 1 \mathrm{H}), 4.52(\mathrm{~d}, J=7 \mathrm{~Hz}, 2 \mathrm{H}), 2.43(\mathrm{~s}, 3 \mathrm{H}), 1.98(\mathrm{q}, J=8 \mathrm{~Hz}, 2 \mathrm{H})$, 1.34 (sextuplet, $J=7 \mathrm{~Hz}, 2 \mathrm{H}), 0.85(\mathrm{t}, J=7 \mathrm{~Hz}, 3 \mathrm{H}) ;{ }^{13} \mathrm{C} \mathrm{NMR}\left(100 \mathrm{MHz}, \mathrm{CDCl}_{3}\right) \delta 155.5,145.9$, $135.9,130.0,129.5,129.3,122.0,62.5,29.2,22.2,21.5,13.4$; IR (neat) 3275 (br), 2959, 2931, 2872, 1740, 1740, 1597, 1460, 1379, 1192, 1179, 1090, 815, 743, 662, $630 \mathrm{~cm}^{-1}$; HMRS (ESI) calc. for $\mathrm{C}_{14} \mathrm{H}_{19} \mathrm{NO}_{5} \mathrm{SNa}[\mathrm{M}+\mathrm{Na}]^{+}:$336.08761; found: 336.08842 .

\section{Characterization of aziridines}

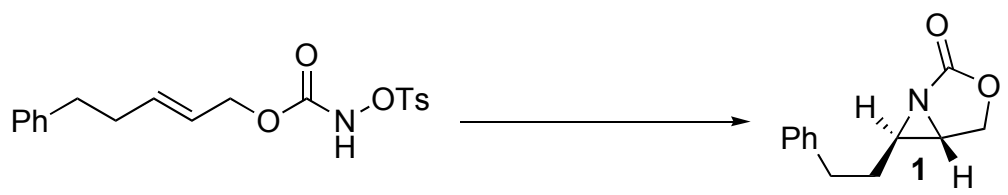

\section{6-Phenethyl-3-oxa-1-aza-bicyclo[3.1.0]hexan-2-one (1)}

The title compound was prepared from (E)-3-phenylprop-2-ene $N$-tosyloxycarbamate (375 mg, 1.00 $\mathrm{mmol}$ ) according to the general procedure $\mathbf{D}$. The desired aziridine (171 $\mathrm{mg}, 84 \%)$ was obtained as a light yellow oil after flash chromatography (30\% AcOEt/hexanes). $\mathrm{R}_{f} 0.44$ (40\% AcOEt/hexanes); ${ }^{1} \mathrm{H}$ NMR (400 MHz, $\left.\mathrm{CDCl}_{3}\right) \delta$ 7.33-7.28 (m, 2H), 7.24-7.19 (m, 3H), 4.38-4.35 (m, 2H), 2.94-2.89 (m, $1 \mathrm{H}), 2.89-2.74(\mathrm{~m}, 2 \mathrm{H}), 2.40-2.34(\mathrm{~m}, 2 \mathrm{H}), 1.94-1.86(\mathrm{~m}, 1 \mathrm{H}) ;{ }^{13} \mathrm{C}\left(100 \mathrm{MHz}, \mathrm{CDCl}_{3}\right) \delta 166.9,140.2$, 
128.5, 128.3, 126.2, 66.5, 46.5, 43.7, 32.8, 32.4; IR (neat) 2920, 1781, 1455, 1372, 1231, 1144, 1102 , $1054,980,814,703 \mathrm{~cm}^{-1}$; HMRS (ESI) calc. for $\mathrm{C}_{12} \mathrm{H}_{13} \mathrm{NO}_{2} \mathrm{Na}[\mathrm{M}+\mathrm{Na}]^{+}$: 226.08385; found: 226.08427.

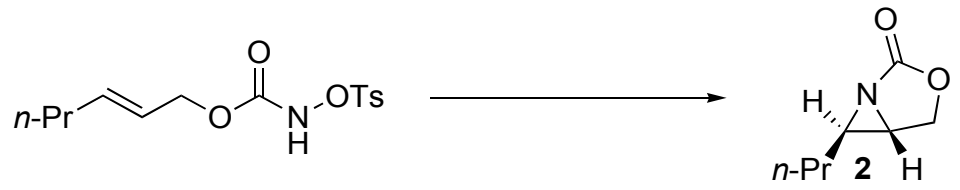

trans-6-Propyl-3-oxa-1-aza-bicyclo[3.1.0]hexan-2-one (2)

The title compound was prepared from $(E)$-hex-2-ene $N$-tosyloxycarbamate $(313 \mathrm{mg}, 1.00 \mathrm{mmol})$ according to the general procedure D. The desired aziridine (93 $\mathrm{mg}, 66 \%)$ was obtained as a light yellow oil after flash chromatography $\left(30 \%\right.$ AcOEt/hexanes-50\% AcOEt/hexanes). $\mathrm{R}_{f} 0.18$ (35\% AcOEt/hexanes); ${ }^{1} \mathrm{H} \mathrm{NMR}\left(400 \mathrm{MHz}, \mathrm{CDCl}_{3}\right) \delta 4.43(\mathrm{dd}, J=9,2 \mathrm{~Hz}, 1 \mathrm{H}), 4.39$ (dd, $\left.J=9,5 \mathrm{~Hz}, 1 \mathrm{H}\right)$, 2.99-2.96 (m, 1H), 2.39-2.35 (m, 1H), 1.60-1.40 (m, 4H), $0.95(\mathrm{t}, J=7 \mathrm{~Hz}, 3 \mathrm{H}) ;{ }^{13} \mathrm{C}(100 \mathrm{MHz}$, $\left.\mathrm{CDCl}_{3}\right) \delta 167.1,66.5,48.2,43.5,32.9,19.5,13.5$; IR (neat) 2960, 2919, 2875, 2850, 1782, 1463, 1375 , 1233, 1142, 1095, 1034, 976, $811 \mathrm{~cm}^{-1}$; HMRS (ESI) calc. for $\mathrm{C}_{7} \mathrm{H}_{11} \mathrm{NO}_{2} \mathrm{Na}[\mathrm{M}+\mathrm{Na}]^{+}$: 164.06820 ; found: 164.06810 .

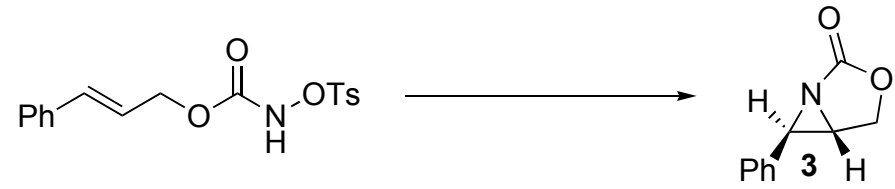

\section{6-Phenyl-3-oxa-1-aza-bicyclo[3.1.0]hexan-2-one (3)}

The title compound was prepared from (E)-3-phenylprop-2-ene $N$ - tosyloxycarbamate (347 mg, 1.00 $\mathrm{mmol}$ ) according to the general procedure D. The desired aziridine (89 $\mathrm{mg}, 51 \%)$ was obtained as a white solid after flash chromatography (30\% AcOEt/hexanes). $\mathrm{R}_{f} 0.43$ (40\% AcOEt/hexanes); mp 106$108{ }^{\circ} \mathrm{C} ;{ }^{1} \mathrm{H}$ NMR $\left(400 \mathrm{MHz}, \mathrm{CDCl}_{3}\right) \delta$ 7.36-7.31 (m, 3H), 7.29-7.25 (m, 2H), 4.61 (dd, $J=10,1 \mathrm{~Hz}$ $1 \mathrm{H}), 4.51(\mathrm{dd}, J=10,5 \mathrm{~Hz}, 1 \mathrm{H}), 3.36(\mathrm{~d}, J=3 \mathrm{~Hz}, 1 \mathrm{H}), 3.24-3.22(\mathrm{~m}, 1 \mathrm{H}) ;{ }^{13} \mathrm{C}\left(100 \mathrm{MHz}, \mathrm{CDCl}_{3}\right) \delta$ 166.5, 134.2, 128.5 (2C), 126.2, 67.1, 48.1, 46.1; IR (neat) 2983, 2916, 1752, 1371, 1146, 1083, 1047, $978,813,754,701,666 \mathrm{~cm}^{-1}$; HMRS (ESI) calc. for $\mathrm{C}_{10} \mathrm{H}_{10} \mathrm{NO}_{2}[\mathrm{M}+\mathrm{H}]^{+}$: 176.07060; found: 176.07059.

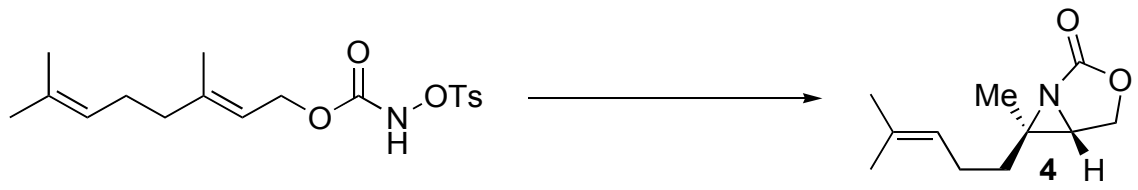

6-Methyl-6-(4-methylpentyl-3-enyl)-3-oxa-1-aza-bicyclo[3.1.0]hexan2-one (4) ${ }^{7}$

Sodium tetra[3,5-di(trifluoromethyl)phenyl]borate $(88 \mathrm{mg}, 0.10 \mathrm{mmol})$ and $\mathrm{CuCl}_{2}\left(\mathrm{Pyridine}_{2}(14 \mathrm{mg}\right.$, $0.050 \mathrm{mmol})$ were dissolved in DCM $(20 \mathrm{~mL})$ and stirred at $25{ }^{\circ} \mathrm{C}$ for $4 \mathrm{~h}$. The green solution was concentrated to dryness, affording a green solid. Acetone $(20 \mathrm{ml})$ was added followed by $\mathrm{K}_{2} \mathrm{CO}_{3}(692$ $\mathrm{mg}, 5.00 \mathrm{mmol}$ ) and (E)-3,7-dimethyl-octa-2,6-diene $N$-tosyloxycarbamate $(367 \mathrm{mg}, 1.00 \mathrm{mmol})$. The mixture was vigorously stirred for $16 \mathrm{~h}$ after which time DCM was added. The solution was filtered over celite $^{\circledR}$ and the filtrate was concentrated. The desired aziridine $(116 \mathrm{mg}, 61 \%)$ was obtained as light yellow oil after flash chromatography (30\% AcOEt/hexanes). $\mathrm{R}_{f} 0.24$ (35\% AcOEt/hexanes); ${ }^{1} \mathrm{H}$ NMR $\left(400 \mathrm{MHz}, \mathrm{CDCl}_{3}\right) \delta 5.08-5.02(\mathrm{~m}, 1 \mathrm{H}), 4.50(\mathrm{dd}, J=10,6 \mathrm{~Hz}, 1 \mathrm{H}), 4.26(\mathrm{dd}, J=10,2 \mathrm{~Hz}, 1 \mathrm{H})$, $3.04(\mathrm{dd}, J=6,2 \mathrm{~Hz}, 1 \mathrm{H}), 2.20-2.00(\mathrm{~m}, 2 \mathrm{H}), 1.68-1.50(\mathrm{~m}, 2 \mathrm{H}), 1.67(\mathrm{~s}, 3 \mathrm{H}), 1.60(\mathrm{~s}, 3 \mathrm{H}), 1.37(\mathrm{~s}$, $3 \mathrm{H}) ;{ }^{13} \mathrm{C}\left(100 \mathrm{MHz}, \mathrm{CDCl}_{3}\right) \delta 165.5,132.6,122.6,64.5,50.9,48.8,38.5,25.5,23.6,17.6,12.0$; IR (neat) $2971,2916,1777,1454,1160,1231,1093,1076,820, \mathrm{~cm}^{-1}$.

\footnotetext{
${ }^{6}$ Bergmeier, S. C.; Stanchina, D. M. J. Org. Chem. 1997, 62, 4449-4456.

7 Kolohang, A.; Stanchina, C. L.; Coates, R. M. Tetrahedron 1999, 55, 9669-9686.
} 


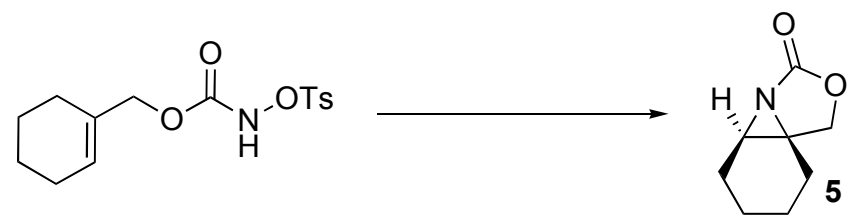

Tetrahydro-2-oxa-3a-azacyclopenta[1,3]cyclopropa[1,2]benzen-3-one $(5)^{8}$

The title compound was prepared from cyclohexene-1-methane $N$-tosyloxycarbamate (327 mg, 1.00 mmol) according to the general procedure D. The desired aziridine (123 $\mathrm{mg}, 79 \%)$ was obtained as a light yellow oil after flash chromatography (30\% AcOEt/hexanes). $\mathrm{R}_{f} 0.21(35 \% \mathrm{AcOEt} / \mathrm{hexanes}) ;{ }^{1} \mathrm{H}$ $\operatorname{NMR}\left(400 \mathrm{MHz}, \mathrm{CDCl}_{3}\right) \delta 4.42(\mathrm{~d}, J=9 \mathrm{~Hz}, 1 \mathrm{H}), 4.06(\mathrm{dd}, J=9,1 \mathrm{~Hz}, 1 \mathrm{H}), 2.70(\mathrm{dd}, J=4,1 \mathrm{~Hz}$, $1 \mathrm{H}), 2.20-2.00(\mathrm{~m}, 2 \mathrm{H}), 1.90-1.77(\mathrm{~m}, 2 \mathrm{H}), 1.60-1.50(\mathrm{~m}, 1 \mathrm{H}), 1.50-1.25(\mathrm{~m}, 3 \mathrm{H}) ;{ }^{13} \mathrm{C}(100 \mathrm{MHz}$, $\left.\mathrm{CDCl}_{3}\right) \delta 167.9,70.2,48.8,48.5,24.7,24.1,20.3,19.1$; IR (neat) 2937, 2865, 1770, 1438, 1219, 1131, $1036,939,631 \mathrm{~cm}^{-1}$.

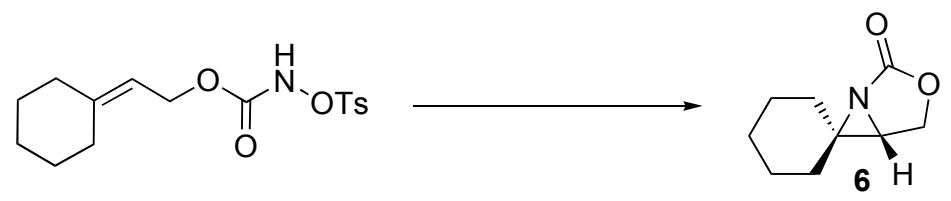

3-Oxa-1-azaspiro[bicyclo[3.1.0]hexane-6,1'-cyclohexan]-2-one (6)

The title compound was prepared from 2-cyclohexylidene $N$-tosyloxycarbamate (339 $\mathrm{mg}, 1.00 \mathrm{mmol}$ ) according to the general procedure D. The desired aziridine $(140 \mathrm{mg}, 84 \%)$ was obtained as a light yellow oil after flash chromatography (30\% AcOEt/hexanes-50\% AcOEt/hexanes). $\mathrm{R}_{f} 0.20$ (40\% AcOEt/hexane); ${ }^{1} \mathrm{H}$ NMR (400 MHz, $\mathrm{CDCl}_{3}$ ) $\delta 4.47$ (dd, $\left.J=10,6 \mathrm{~Hz}, 1 \mathrm{H}\right), 4.25(\mathrm{dd}, J=10,2 \mathrm{~Hz}, 1 \mathrm{H})$, $3.00(\mathrm{dd}, J=6,2 \mathrm{~Hz}, 1 \mathrm{H}), 1.82-1.63(\mathrm{~m}, 4 \mathrm{H}), 1.60-1.47(\mathrm{~m}, 6 \mathrm{H}) ;{ }^{13} \mathrm{C}$ NMR $\left(100 \mathrm{MHz}, \mathrm{CDCl}_{3}\right) \delta 165.4$, 64.2, 53.6, 49.3, 35.3, 25.1, 25.0, 24.2, 24.1; IR (neat) 2934, 2859, 1760, 1449, 1372, 1160, 1146, 1087, 1050, 1001, $819 \mathrm{~cm}^{-1}$; HMRS (ESI) calc. for $\mathrm{C}_{9} \mathrm{H}_{13} \mathrm{NO}_{2} \mathrm{Na}[\mathrm{M}+\mathrm{Na}]^{+}$: 190.08385; found: 190.08397.

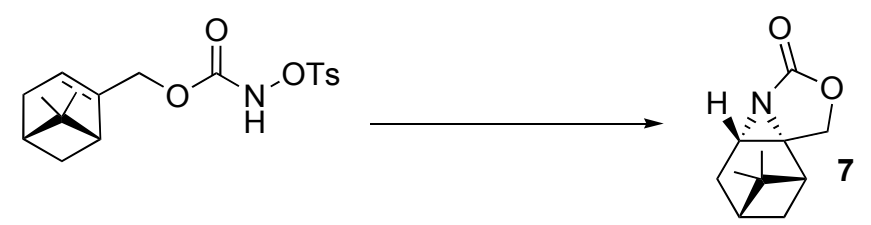

$(1 R, 2 R, 7 S, 9 R)-10,10-D i m e t h y l-4-0 x a-6-a z a t e t r a c y c l o[7.1 .1 .02,6.02,7]$ undecan-5-one (7)

The title compound was prepared from 2-methyl-6,6-dimethyl-bicyclo[3.1.1]hept-2-ene $N$ tosyloxycarbamate $(365 \mathrm{mg}, 1.00 \mathrm{mmol})$ according to the general procedure D using $5 \mathrm{~mol} \%$ of $\mathrm{Cu}$ (pyridine $)_{4}(\mathrm{OTf})_{2}$. The desired aziridine $(151 \mathrm{mg}, 78 \%)$ was obtained as white solid after flash chromatography (30\% AcOEt/hexanes). $\mathrm{R}_{f} 0.32(\mathrm{DCM}) ; \mathrm{mp} 118-121{ }^{\circ} \mathrm{C} ;[\alpha]_{\mathrm{D}}{ }^{25}=-8.3(c 1.00, \mathrm{DCM})$; ${ }^{1} \mathrm{H}$ NMR $\left(300 \mathrm{MHz}, \mathrm{CDCl}_{3}\right) \delta 4.46(\mathrm{~d}, J=9 \mathrm{~Hz}, 1 \mathrm{H}), 4.15(\mathrm{~d}, J=9 \mathrm{~Hz}, 1 \mathrm{H}), 2.75(\mathrm{~d}, J=5 \mathrm{~Hz}, 1 \mathrm{H})$, 2.26-2.16 (m, 2H), 2.15-2.05 (m, 2H), 1.98-1.81 (m, 1H), $1.77(\mathrm{~d}, J=10 \mathrm{~Hz}, 1 \mathrm{H}), 1.31(\mathrm{~s}, 3 \mathrm{H}), 0.98(\mathrm{~s}$, $3 \mathrm{H}) ;{ }^{13} \mathrm{C}$ NMR $\left(75 \mathrm{MHz}, \mathrm{CDCl}_{3}\right) \delta 166.5,70.3,53.1,45.7,41.6,40.5,40.1,27.0,26.5,25.6,20.4$; IR (neat) 2993, 2972, 2941, 1784, 1140, $1043 \mathrm{~cm}^{-1}$; HMRS (ESI) calc. for $\mathrm{C}_{11} \mathrm{H}_{16} \mathrm{NO}_{2}[\mathrm{M}+\mathrm{H}]^{+}:$194.11756; found: 194.11736.

8 Padwa, A.; Flick, A. C.; Leverett, C. A.; Stengel, T. J. Org. Chem. 2004, 69, 6377-6386. 

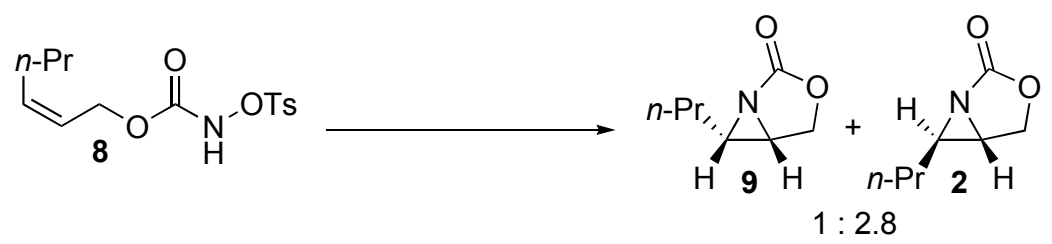

cis-6-Propyl-3-oxa-1-aza-bicyclo[3.1.0]hexan-2-one (9) ${ }^{6}$

The title compound was prepared from $(Z)$-hex-2-ene $N$-tosyloxycarbamate $(313 \mathrm{mg}, 1.00 \mathrm{mmol})$ according to the general procedure $\mathbf{D}$. The desired aziridine was obtained as a mixture of diastereomers (cis:trans 1:2.8) and as a light yellow oil (77 mg, 55\%) after flash chromatography (30\% AcOEt/hexanes-50\% AcOEt/hexanes). After further purification, a pure sample of the cis aziridine was obtained. $\mathrm{R}_{f} 0.16$ (35\% AcOEt/hexanes); ${ }^{1} \mathrm{H}$ NMR $\left(400 \mathrm{MHz}, \mathrm{CDCl}_{3}\right) \delta 4.54$ (dd, $\left.J=10,6 \mathrm{~Hz}, 1 \mathrm{H}\right)$, $4.27(\mathrm{dd}, J=10,2 \mathrm{~Hz}, 1 \mathrm{H}), 3.22-3.19(\mathrm{~m}, 1 \mathrm{H}), 2.73(\mathrm{dd}, J=9,3 \mathrm{~Hz}, 1 \mathrm{H}), 1.70-1.45(\mathrm{~m}, 4 \mathrm{H}), 1.00(\mathrm{t}, J$ $=7 \mathrm{~Hz}, 3 \mathrm{H}) ;{ }^{13} \mathrm{C}$ NMR $\left(100 \mathrm{MHz}, \mathrm{CDCl}_{3}\right) \delta 165.3,64.1,45.7,42.6,25.3,20.1,13.7$; IR (neat) 2962, $2874,1769,1372,1262,1167,1137,1088,1031 \mathrm{~cm}^{-1}$; HMRS (ESI) calc. for $\mathrm{C}_{7} \mathrm{H}_{11} \mathrm{NO}_{2} \mathrm{Na}[\mathrm{M}+\mathrm{Na}]^{+}$: 164.06820; found: 164.06839 .

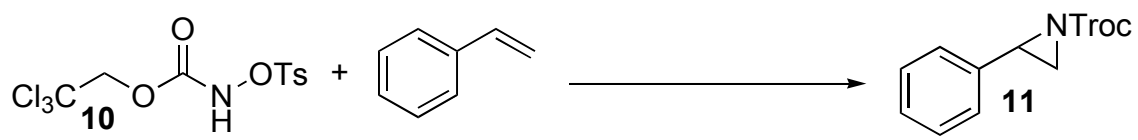

\section{2,2,2-Trichloroethyl 2-phenylaziridine-1-carboxylate (11)}

The title compound was prepared from $10(182 \mathrm{mg}, 0.500 \mathrm{mmol})$ and styrene $(290 \mu \mathrm{L}, 2.50 \mathrm{mmol})$ according to the general procedure E. The desired aziridine (108 $\mathrm{mg}, 74 \%)$ was obtained as a white solid after flash chromatography (1\% ether/hexanes-10\% ether/hexanes). $\quad \mathrm{R}_{f} \quad 0.42 \quad(15 \%$ EtOAc/hexanes); mp 71-72 ${ }^{\circ} \mathrm{C} ;{ }^{1} \mathrm{H}$ NMR $\left(300 \mathrm{MHz}, \mathrm{CDCl}_{3}\right) \delta$ 7.46-7.27 (m, 5H), $4.84(\mathrm{~d}, J=12 \mathrm{~Hz}$, $1 \mathrm{H}), 4.74(\mathrm{~d}, J=12 \mathrm{~Hz}, 1 \mathrm{H}), 3.64(\mathrm{dd}, J=6,4 \mathrm{~Hz}, 1 \mathrm{H}), 2.82(\mathrm{~d}, J=6 \mathrm{~Hz}, 1 \mathrm{H}), 2.44(\mathrm{~d}, J=4 \mathrm{~Hz}, 1 \mathrm{H})$; ${ }^{13} \mathrm{C}$ NMR $\left(75 \mathrm{MHz}, \mathrm{CDCl}_{3}\right) \delta 161.3,136.1,128.5,128.0,126.1,94.8,75.3,39.7,35.1$; IR (neat) 3004 , $1735,1170,907,731 \mathrm{~cm}^{-1}$; HMRS (ESI) calc. for $\mathrm{C}_{11} \mathrm{H}_{11} \mathrm{Cl}_{3} \mathrm{NO}_{2}[\mathrm{M}+\mathrm{H}]^{+}: 293.9849$; found 293.9857.

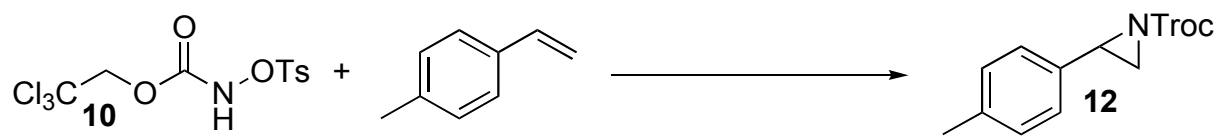

\section{2,2,2-Trichloroethyl 2-p-tolylaziridine-1-carboxylate (12)}

The title compound was prepared from $10(182 \mathrm{mg}, 0.500 \mathrm{mmol})$ and 1-methyl-4-vinylbenzene (330 $\mu \mathrm{L}, 2.50 \mathrm{mmol})$ according to the general procedure E. The desired aziridine $(112 \mathrm{mg}, 68 \%)$ was obtained as a colorless oil after flash chromatography (hexanes-5\% ether/hexanes). $\mathrm{R}_{f} 0.53$ (15\% EtOAc/hexanes); mp 49-50 ${ }^{\circ} \mathrm{C}$; ${ }^{1} \mathrm{H}$ NMR $\left(400 \mathrm{MHz}, \mathrm{C}_{6} \mathrm{D}_{6}\right) \delta 7.07(\mathrm{~d}, J=8 \mathrm{~Hz}, 2 \mathrm{H}), 6.91(\mathrm{~d}, J=8 \mathrm{~Hz}$, $2 \mathrm{H}) ; 4.53(\mathrm{~d}, J=12 \mathrm{~Hz}, 1 \mathrm{H}), 4.42(\mathrm{~d}, J=12 \mathrm{~Hz}, 1 \mathrm{H}), 3.27(\mathrm{dd}, J=6,4 \mathrm{~Hz}, 1 \mathrm{H}), 2.32(\mathrm{~d}, J=6 \mathrm{~Hz}$, $1 \mathrm{H}), 2.06(\mathrm{~s}, 3 \mathrm{H}), 1.95(\mathrm{~d}, J=4 \mathrm{~Hz}, 1 \mathrm{H}) ;{ }^{13} \mathrm{C}$ NMR $\left(100 \mathrm{MHz}, \mathrm{C}_{6} \mathrm{D}_{6}\right) \delta$ 161.4, 137.8, 134.0, 129.5, 126.6, 95.7, 75.4, 40.0, 35.2, 21.1; IR (neat) 3001, 2954, 1733, 1391, 1296, 1277, 1165, 817, $718 \mathrm{~cm}^{-1}$; HMRS (ESI) calc. for $\mathrm{C}_{12} \mathrm{H}_{13} \mathrm{Cl}_{3} \mathrm{NO}_{2}[\mathrm{M}+\mathrm{H}]^{+}$: 308.006; found 308.0012.

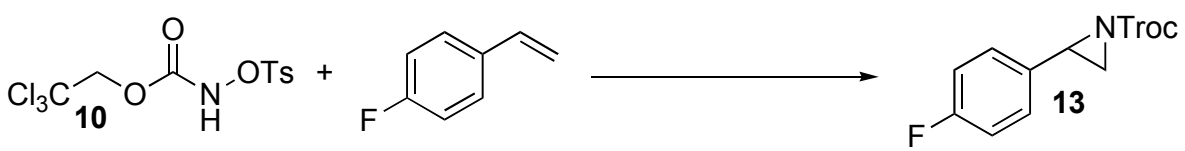

\section{2,2,2-Trichloroethyl 2-(4-fluorophenyl)aziridine-1-carboxylate (13)}

The title compound was prepared from $10(182 \mathrm{mg}, 0.500 \mathrm{mmol})$ and 1-fluoro-4-vinylbenzene (300 $\mu \mathrm{L}, 2.50 \mathrm{mmol}$ ) according to the general procedure $\mathbf{E}$. The desired aziridine $(86 \mathrm{mg}, 55 \%)$ was obtained as colorless oil after flash chromatography ( $2 \%$ ether/hexanes- $10 \%$ ether/hexanes). $\mathrm{R}_{f} 0.32$ (15\% EtOAc/hexanes); ${ }^{1} \mathrm{H}$ NMR (400 MHz, $\mathrm{CDCl}_{3}$ ) $\delta$ 7.31-7.28 (m, 2H), 7.06-7.02 (m, 2H), 4.83 (d, $J$ 
$=12 \mathrm{~Hz}, 1 \mathrm{H}), 4.75(\mathrm{~d}, J=12 \mathrm{~Hz}, 1 \mathrm{H}), 3.61(\mathrm{dd}, J=6,4 \mathrm{~Hz}, 1 \mathrm{H}), 2.81(\mathrm{~d}, J=6 \mathrm{~Hz}, 1 \mathrm{H}), 2.39(\mathrm{~d}, J=4$ $\mathrm{Hz}, 1 \mathrm{H}) ;{ }^{13} \mathrm{C} \mathrm{NMR}\left(100 \mathrm{MHz}, \mathrm{CDCl}_{3}\right) \delta 162.5(\mathrm{~d}, J=250 \mathrm{~Hz}), 161.2,131.9(\mathrm{~d}, J=3 \mathrm{~Hz}) ; 127.8(\mathrm{~d}, J=$ $8 \mathrm{~Hz}), 115.5(\mathrm{~d}, J=22 \mathrm{~Hz}), 94.7,75.3,39.1,35.1 ;{ }^{19} \mathrm{~F} \mathrm{NMR}\left(282 \mathrm{MHz}, \mathrm{CDCl}_{3}\right) \delta-261.6(\mathrm{tt}, J=9,5$ $\mathrm{Hz}, 1 \mathrm{~F}$ ); IR (neat) 3003, 2956, 1733, 1513, 1389, 1156, 818, $715 \mathrm{~cm}^{-1}$; HMRS (ESI) calc. for $\mathrm{C}_{11} \mathrm{H}_{10} \mathrm{Cl}_{3} \mathrm{FNO}_{2}[\mathrm{M}+\mathrm{H}]^{+}:$311.97557; found 311.97704 .

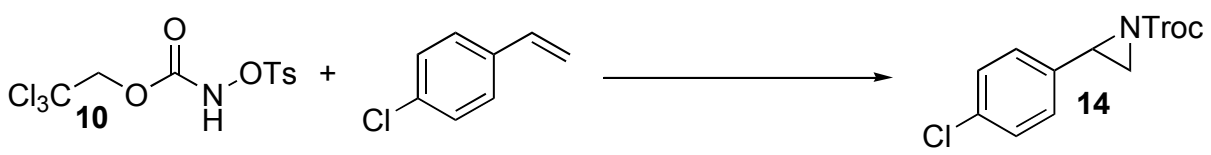

\section{2,2,2-Trichloroethyl 2-(4-chlorophenyl)aziridine-1-carboxylate (14)}

The title compound was prepared from $10(182 \mathrm{mg}, 0.500 \mathrm{mmol})$ and 1-chloro-4-vinylbenzene (300 $\mu \mathrm{L}, 2.50 \mathrm{mmol})$ according to the general procedure $\mathbf{E}$. The desired aziridine $(101 \mathrm{mg}, 62 \%)$ was obtained as colorless oil after flash chromatography (2\% ether/hexanes-10\% ether/hexanes). $\mathrm{R}_{f} 0.27$ (15\% EtOAc/hexanes); ${ }^{1} \mathrm{H}$ NMR (400 MHz, $\left.\mathrm{CDCl}_{3}\right) \delta 7.34(\mathrm{~d}, J=8 \mathrm{~Hz}, 2 \mathrm{H}), 7.28(\mathrm{~d}, J=8 \mathrm{~Hz}, 2 \mathrm{H})$, $4.85(\mathrm{~d}, J=12 \mathrm{~Hz}, 1 \mathrm{H}), 4.77(\mathrm{~d}, J=12 \mathrm{~Hz}, 1 \mathrm{H}), 3.62(\mathrm{dd}, J=6,3.5 \mathrm{~Hz}, 1 \mathrm{H}), 2.84(\mathrm{~d}, J=6 \mathrm{~Hz}, 1 \mathrm{H})$, $2.40(\mathrm{~d}, J=3.5 \mathrm{~Hz}, 1 \mathrm{H}) ;{ }^{13} \mathrm{C}$ NMR $\left(100 \mathrm{MHz}, \mathrm{CDCl}_{3}\right) \delta 161.1,134.7,133.8,128.6,127.5,94.7,75.3$, 39.0, 35.2; IR (neat) 3000, 2955, 1738, 1495, 1388, 1308, 1290, 1168, 1091, 824, $720 \mathrm{~cm}^{-1}$; HMRS (ESI) calc. for $\mathrm{C}_{11} \mathrm{H}_{10} \mathrm{Cl}_{4} \mathrm{NO}_{2}[\mathrm{M}+\mathrm{H}]^{+}$: 327.94602; found 327.94584 .

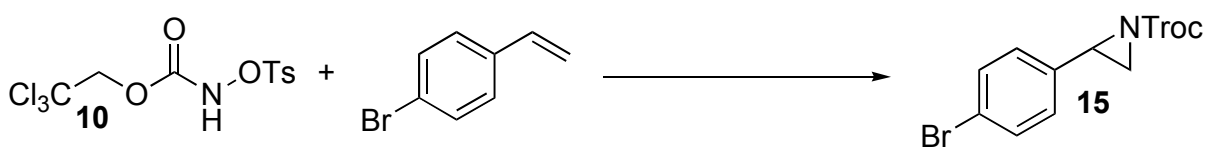

\section{2,2,2-Trichloroethyl 2-(4-bromophenyl)aziridine-1-carboxylate (15)}

The title compound was prepared from $10(182 \mathrm{mg}, 0.500 \mathrm{mmol})$ and 1-bromo-4-vinylbenzene (320 $\mu \mathrm{L}, 2.50 \mathrm{mmol})$ according to the general procedure $\mathbf{E}$. The desired aziridine $(105 \mathrm{mg}, 56 \%)$ was obtained as colorless oil after flash chromatography (2\% ether/hexanes-10\% ether/hexanes). $\mathrm{R}_{f} 0.29$ (15\% EtOAc/hexanes); ${ }^{1} \mathrm{H}$ NMR (400 MHz, $\left.\mathrm{CDCl}_{3}\right) \delta 7.48(\mathrm{~d}, J=8 \mathrm{~Hz}, 2 \mathrm{H}), 7.20(\mathrm{~d}, J=8 \mathrm{~Hz}, 2 \mathrm{H})$, $4.82(\mathrm{~d}, J=12 \mathrm{~Hz}, 1 \mathrm{H}), 4.75(\mathrm{~d}, J=12 \mathrm{~Hz}, 1 \mathrm{H}), 3.58(\mathrm{dd}, J=6,4 \mathrm{~Hz}, 1 \mathrm{H}), 2.82(\mathrm{~d}, J=6 \mathrm{~Hz}, 1 \mathrm{H}), 2.38$ $(\mathrm{d}, J=4 \mathrm{~Hz}, 1 \mathrm{H}) ;{ }^{13} \mathrm{C}$ NMR $\left(100 \mathrm{MHz}, \mathrm{CDCl}_{3}\right) \delta 161.1,135.3,131.6,127.8,122.0,94.7,75.3,39.1$, 35.2; IR (neat) 3000, 2954, 1737, 1385, 1307, 1290, 1167, 1070, 1011, 821, $719 \mathrm{~cm}^{-1}$; HMRS (ESI) calc. for $\mathrm{C}_{11} \mathrm{H}_{10} \mathrm{BrCl}_{3} \mathrm{NO}_{2}[\mathrm{M}+\mathrm{H}]^{+}$: 371.8965; found 371.8968 .

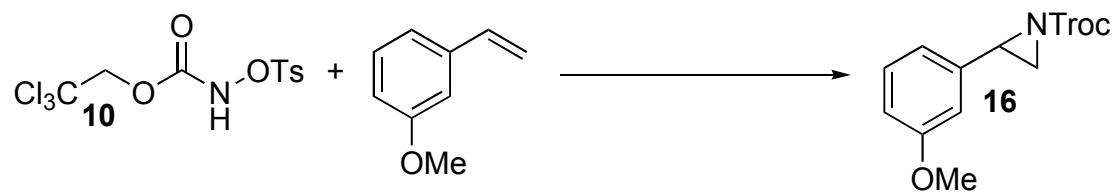

\section{2,2,2-Trichloroethyl 2-(3-methoxyphenyl)aziridine-1-carboxylate (16)}

The title compound was prepared from $10(182 \mathrm{mg}, 0.500 \mathrm{mmol})$ and 3-methoxy-4-vinylbenzene (350 $\mu \mathrm{L}, 2.50 \mathrm{mmol})$ according to the general procedure E. The desired aziridine (95 $\mathrm{mg}, 62 \%)$ was obtained as colorless oil after flash chromatography (2\% ether/hexanes-15\% ether/hexanes). $\mathrm{R}_{f} 0.21$ (15\% EtOAc/hexanes); ${ }^{1} \mathrm{H}$ NMR (400 MHz, $\left.\mathrm{C}_{6} \mathrm{D}_{6}\right) \delta 6.98(\mathrm{t}, J=8 \mathrm{~Hz}, 1 \mathrm{H}), 6.83-6.79(\mathrm{~m}, 1 \mathrm{H}), 6.77-$ $6.74(\mathrm{~m}, 1 \mathrm{H}), 6.65(\mathrm{ddd}, J=8,3,1 \mathrm{~Hz}, 1 \mathrm{H}), 4.48(\mathrm{~d}, J=12 \mathrm{~Hz}, 1 \mathrm{H}), 4.40(\mathrm{~d}, J=12 \mathrm{~Hz}, 1 \mathrm{H}), 3.23$ (s, $3 \mathrm{H}), 3.22-3.20(\mathrm{~m}, 1 \mathrm{H}), 2.27(\mathrm{dd}, J=6,0.5 \mathrm{~Hz}, 1 \mathrm{H}), 1.89(\mathrm{dd}, J=4,0.5 \mathrm{~Hz}, 1 \mathrm{H}) ;{ }^{13} \mathrm{C} \mathrm{NMR}(100 \mathrm{MHz}$, $\left.\mathrm{C}_{6} \mathrm{D}_{6}\right) \delta 161.3,160.5,138.5,129.9,118.9,114.1,111.9,95.7,75.4,54.8,40.0,35.3$; IR (neat) 3002, 2955, 2837, 1738, 1604, 1400, 1302, 1204, $1181 \mathrm{~cm}^{-1}$; HMRS (ESI) calc. for $\mathrm{C}_{12} \mathrm{H}_{13} \mathrm{Cl}_{3} \mathrm{NO}_{2}[\mathrm{M}+\mathrm{H}]^{+}$: 323.9955; found 323.9963. 


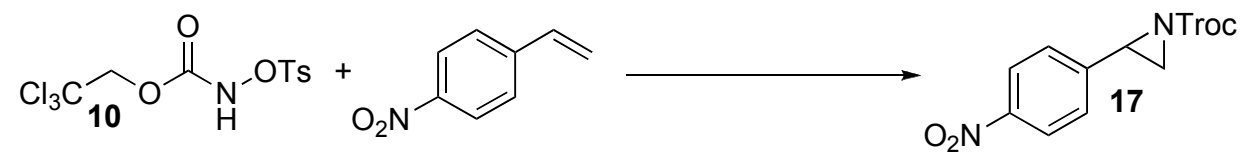

\section{2,2,2-Trichloroethyl 2-(4-nitrophenyl)aziridine-1-carboxylate (17)}

The title compound was prepared according to the general procedure E. The desired aziridine (86 mg, $51 \%$ ) was obtained as a yellow oil after flash chromatography (15\% AcOEt/hexanes). $\mathrm{R}_{f} 0.13(15 \%$ EtOAc/hexanes); ${ }^{1} \mathrm{H}$ NMR (400 MHz, $\left.\mathrm{CDCl}_{3}\right) \delta 8.15(\mathrm{~d}, J=9 \mathrm{~Hz}, 2 \mathrm{H}), 7.51(\mathrm{~d}, J=9 \mathrm{~Hz}, 2 \mathrm{H}), 4.84(\mathrm{~d}$, $J=12 \mathrm{~Hz}, 1 \mathrm{H}), 4.78(\mathrm{~d}, J=12 \mathrm{~Hz}, 1 \mathrm{H}), 3.71(\mathrm{dd}, J=6,3 \mathrm{~Hz}, 1 \mathrm{H}), 2.91(\mathrm{~d}, J=6 \mathrm{~Hz}, 1 \mathrm{H}), 2.41(\mathrm{~d}, J$ $=3 \mathrm{~Hz}, 1 \mathrm{H}) ;{ }^{13} \mathrm{C}$ NMR $\left(100 \mathrm{MHz}, \mathrm{C}_{6} \mathrm{D}_{6}\right) \delta 160.8,147.6,143.7,127.0,123.8,94.6,75.4,38.6,35.8$; IR (neat) 2956, 1738, 1605, 1520, 1346, $1169 \mathrm{~cm}^{-1}$; HMRS (MAB) calcd for $\mathrm{C}_{11} \mathrm{H}_{10} \mathrm{Cl}_{3} \mathrm{~N}_{2} \mathrm{O}_{4}[\mathrm{M}+\mathrm{H}]^{+}$: 338.97007; found 338.96910.

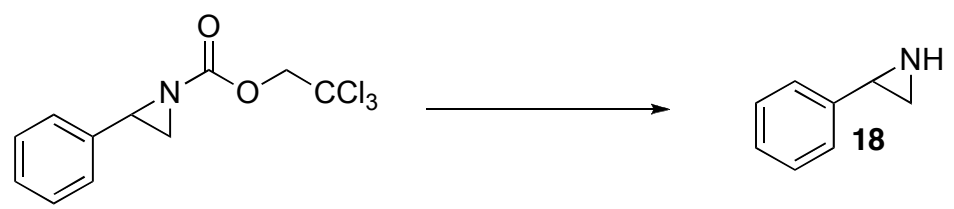

\section{2-Phenylaziridine (18). ${ }^{9}$}

To a solution of 2,2,2-trichloroethyl 2-phenylaziridine-1-carboxylate (146 $\mathrm{mg}, 0.500 \mathrm{mmol})$ in mixture of acetonitrile $(5 \mathrm{~mL})$ methanol $(2 \mathrm{~mL})$ was added $\mathrm{LiOH} \bullet \mathrm{H}_{2} \mathrm{O}(105 \mathrm{mg}, 2.50 \mathrm{mmol})$ and water $(0.090$ $\mathrm{mL}, 5.0 \mathrm{mmol})$. After 5 hours of stirring at $23{ }^{\circ} \mathrm{C}, \mathrm{H}_{2} \mathrm{O}(5 \mathrm{~mL})$ and AcOEt $(15 \mathrm{~mL})$ were added and the two layers were separated. The aqueous layer was washed with AcOEt $(3 \mathrm{x} 15 \mathrm{~mL})$. The combined organic layers were washed with brine $(20 \mathrm{~mL})$, dried over $\mathrm{MgSO}_{4}$ and concentrated to give the desired aziridine 18 (44 mg, 74\%) as a colorless oil after flash chromatography (AcOEt). $\mathrm{R}_{f} 0.35$ (100\% AcOEt). ${ }^{1} \mathrm{H}$ NMR (400 MHz, $\left.\mathrm{C}_{6} \mathrm{D}_{6}\right) \delta 7.30-7.00(\mathrm{~m}, 5 \mathrm{H}), 2.46(\mathrm{~m}, 1 \mathrm{H}), 1.58(\mathrm{~m}, 1 \mathrm{H}), 1.34(\mathrm{~m}, 1 \mathrm{H})$, 0.15 (br, 1H); ${ }^{13} \mathrm{C}$ NMR (100 MHz, $\left.\mathrm{CDCl}_{3}\right) \delta 140.3,128.4,127.0,125.6,32.1,29.2$.

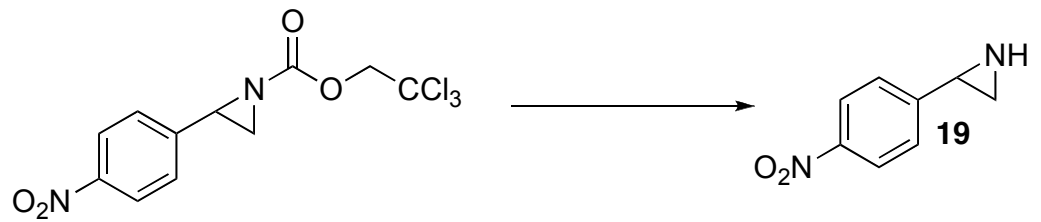

\section{2-(4-nitrophenyl)aziridine (19).}

To a solution of 2,2,2-trichloroethyl 2-(4-nitrophenyl)aziridine-1-carboxylate (167 mg, $0.500 \mathrm{mmol})$ in mixture of acetonitrile $(5 \mathrm{~mL})$ methanol $(2 \mathrm{~mL})$ was added $\mathrm{LiOH}_{2} \mathrm{H}(105 \mathrm{mg}, 2.50 \mathrm{mmol})$ and water $(0.090 \mathrm{~mL}, 5.0 \mathrm{mmol})$. After 5 hours of stirring at $23{ }^{\circ} \mathrm{C}, \mathrm{H}_{2} \mathrm{O}(5 \mathrm{~mL})$ and AcOEt $(15 \mathrm{~mL})$ were added and the two layers were separated. The aqueous layer was washed with AcOEt $(3 \mathrm{x} 15 \mathrm{~mL})$. The combined organic layers were washed with brine $(20 \mathrm{~mL})$, dried over $\mathrm{MgSO}_{4}$ and concentrated to give the desired aziridine 19 (74 $\mathrm{mg}, 91 \%)$ as yellow oil after flash chromatography (100\% AcOEt). $\mathrm{R}_{f} 0.33$ (AcOEt). ${ }^{1} \mathrm{H}$ NMR $\left(400 \mathrm{MHz}, \mathrm{CDCl}_{3}\right) \delta 8.16(\mathrm{~d}, J=9 \mathrm{~Hz}, 2 \mathrm{H}), 7.40(\mathrm{~d}, J=9 \mathrm{~Hz}, 2 \mathrm{H}), 3.14(\mathrm{~d}, J=3$ $\mathrm{Hz}, 1 \mathrm{H}), 2.37(\mathrm{~d}, J=6 \mathrm{~Hz}, 1 \mathrm{H}), 1.73(\mathrm{~s}, 1 \mathrm{H}), 1.14(\mathrm{br}, 1 \mathrm{H}) ;{ }^{13} \mathrm{C}$ NMR $\left(100 \mathrm{MHz}, \mathrm{CDCl}_{3}\right) \delta 148.5$, 147.0, 126.4, 123.6, 31.2, 30.5. IR (neat) 3312, 3064, 2992, 1601, 1515, 1344, 1109, 851 cm ${ }^{-1}$ HMRS (MAB) calcd for $\mathrm{C}_{8} \mathrm{H}_{10} \mathrm{~N}_{2} \mathrm{O}_{2}[\mathrm{M}+\mathrm{H}]^{+}:$165.06585; found 165.06598 .

\footnotetext{
${ }^{9}$ Alonso, D. A.; Andersson, P. G. J. Org. Chem. 1998, 63, 9455-9461.
} 

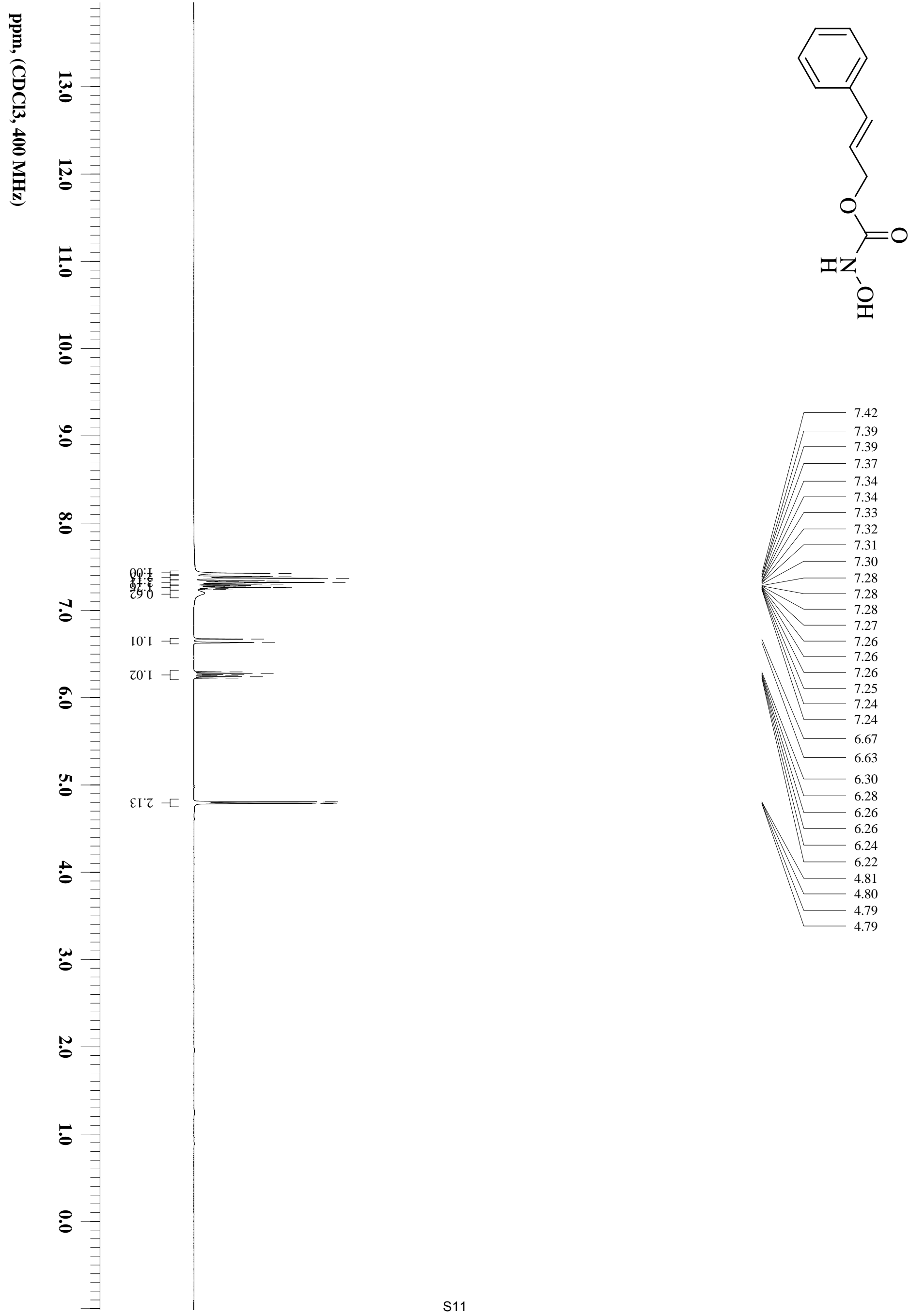

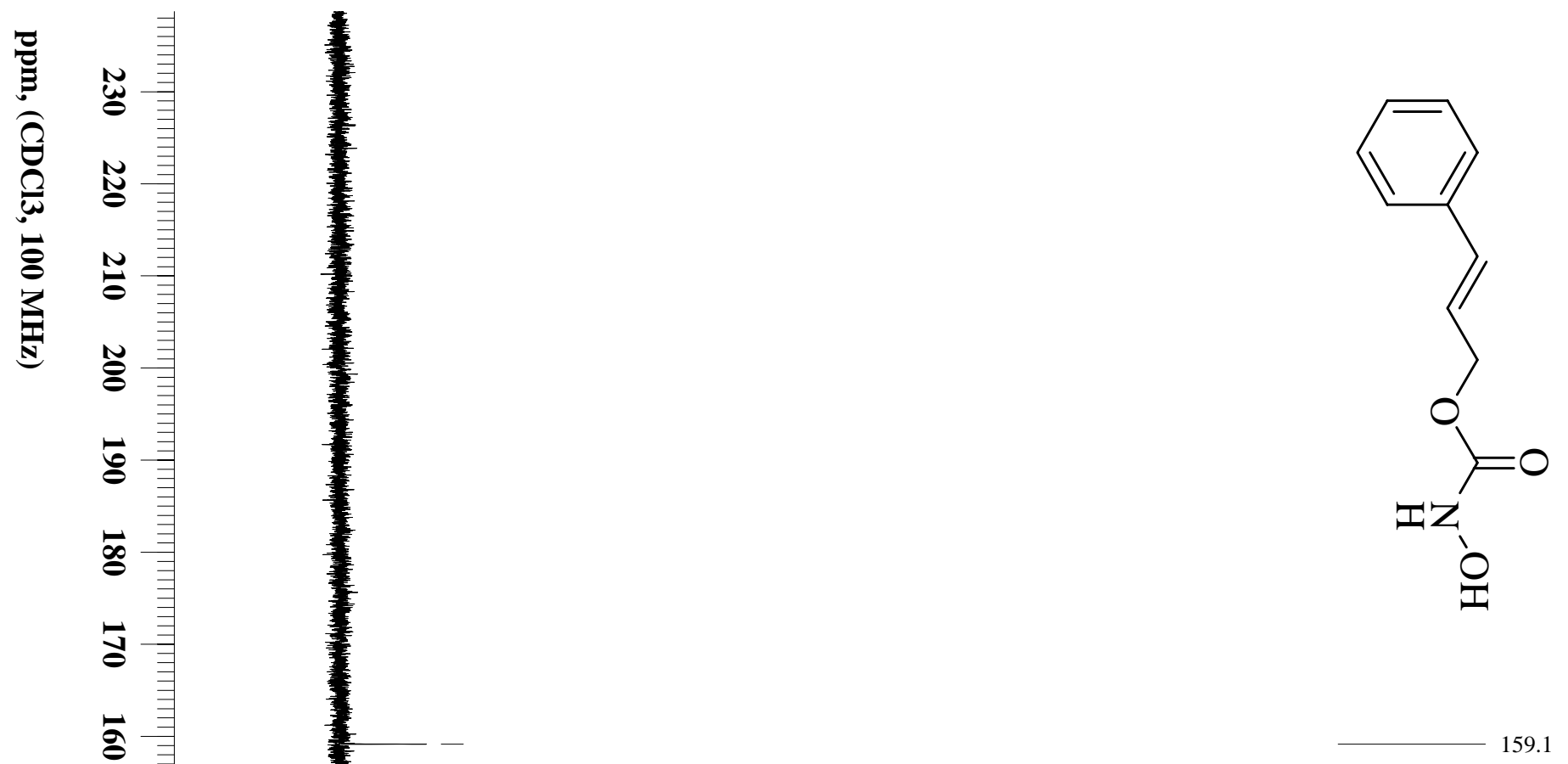

동

E

๘

్ㅡㅇ

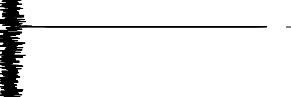

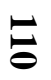

s

8

\&

¿

8

등

ह

ש్



웅

○录

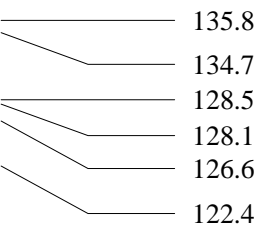

66.7 


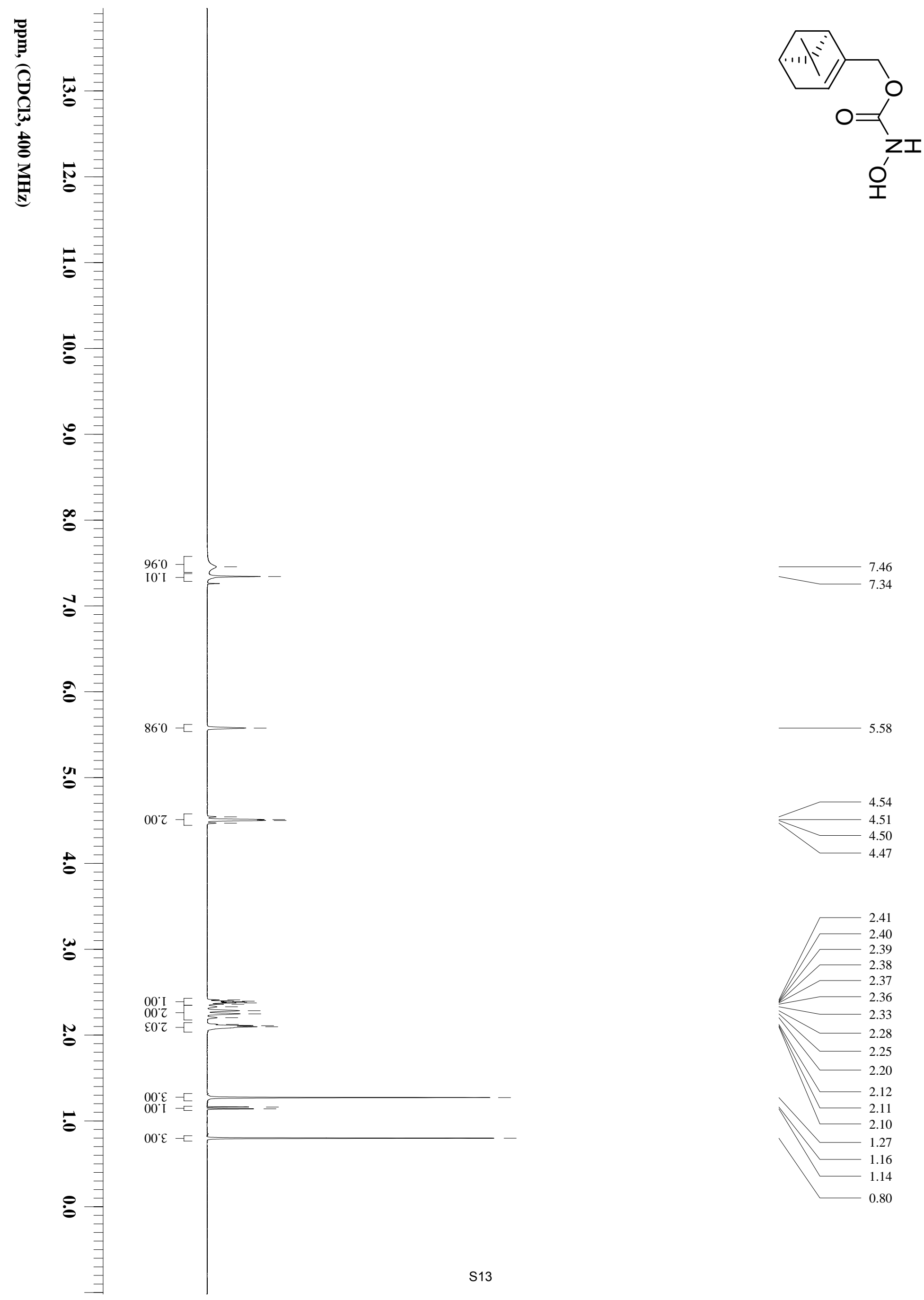




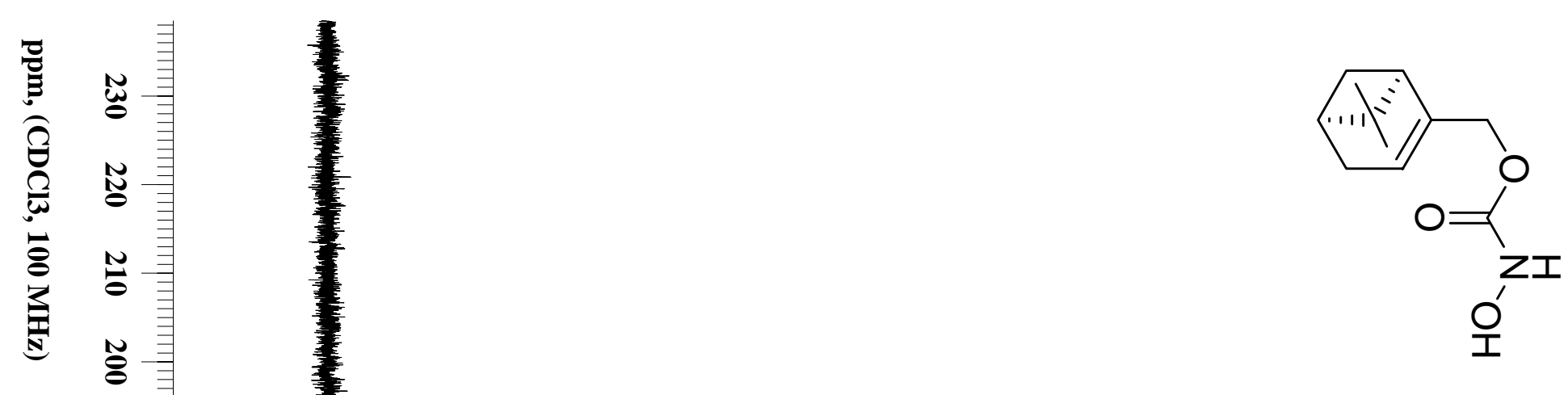

8

5

홍

159.3

동

昰

๘

몽

క

s

8

$\infty$

$d$

8

둉

ह

.

$\ddot{\sigma}$

ㄴ.

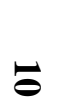

0

○严 


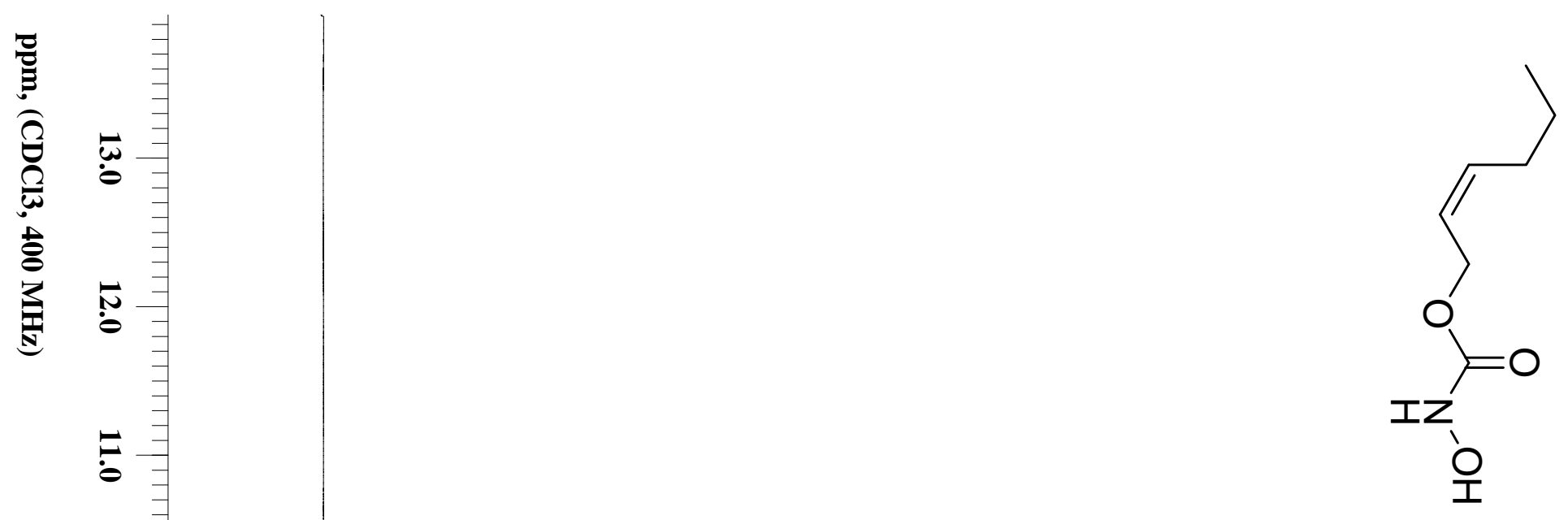

:

0

$007-$

늠

$86: 0-\square=$

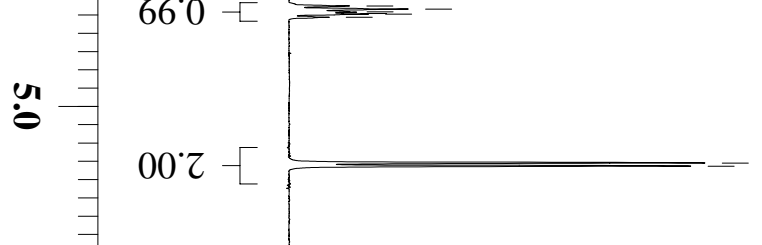

2.09

2.07

2.05

2.03

1.39

1.37

เ

I0 $7-\square$

$\succ 0^{\circ} \varepsilon-\sqsubset$ 


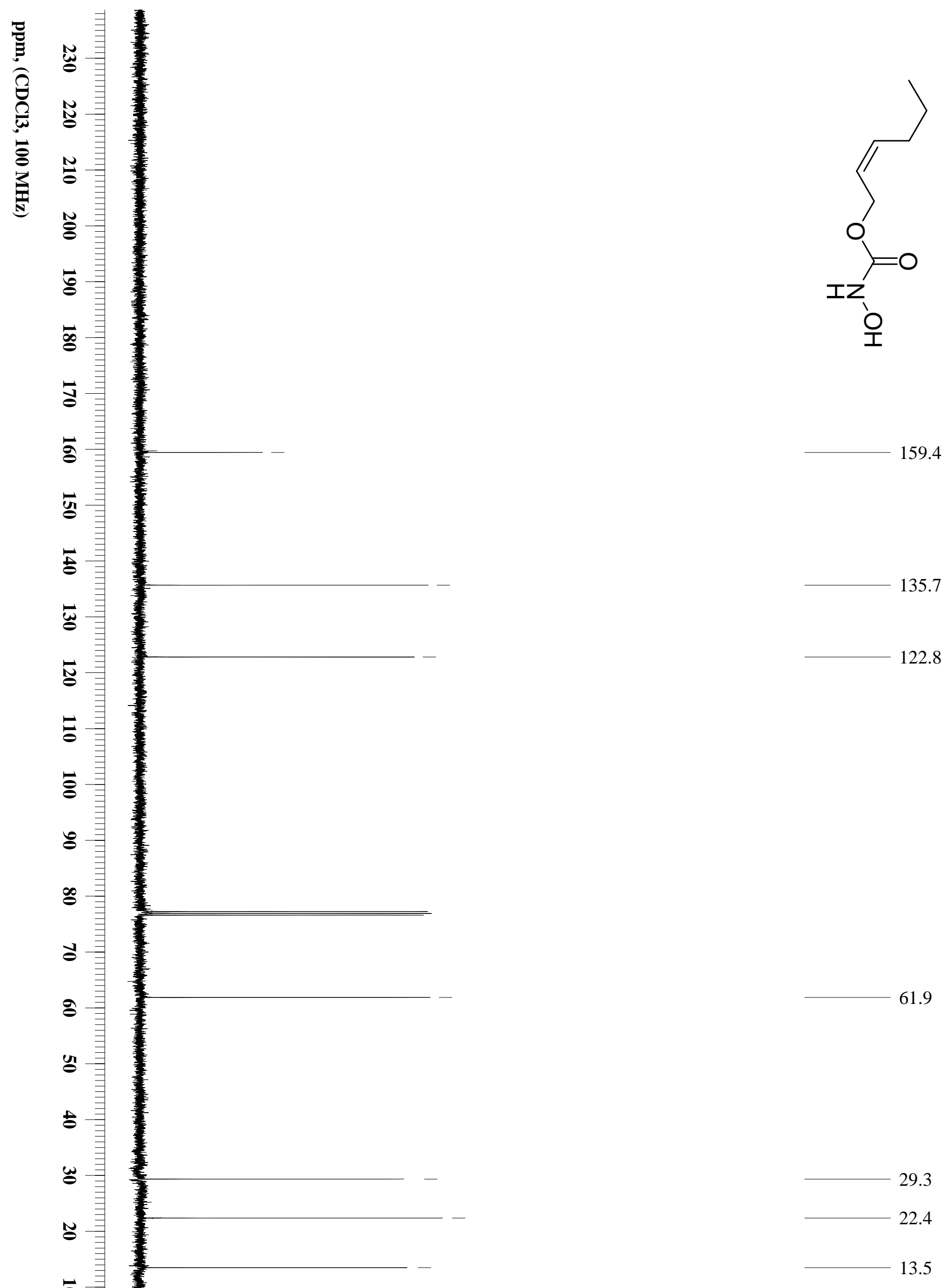




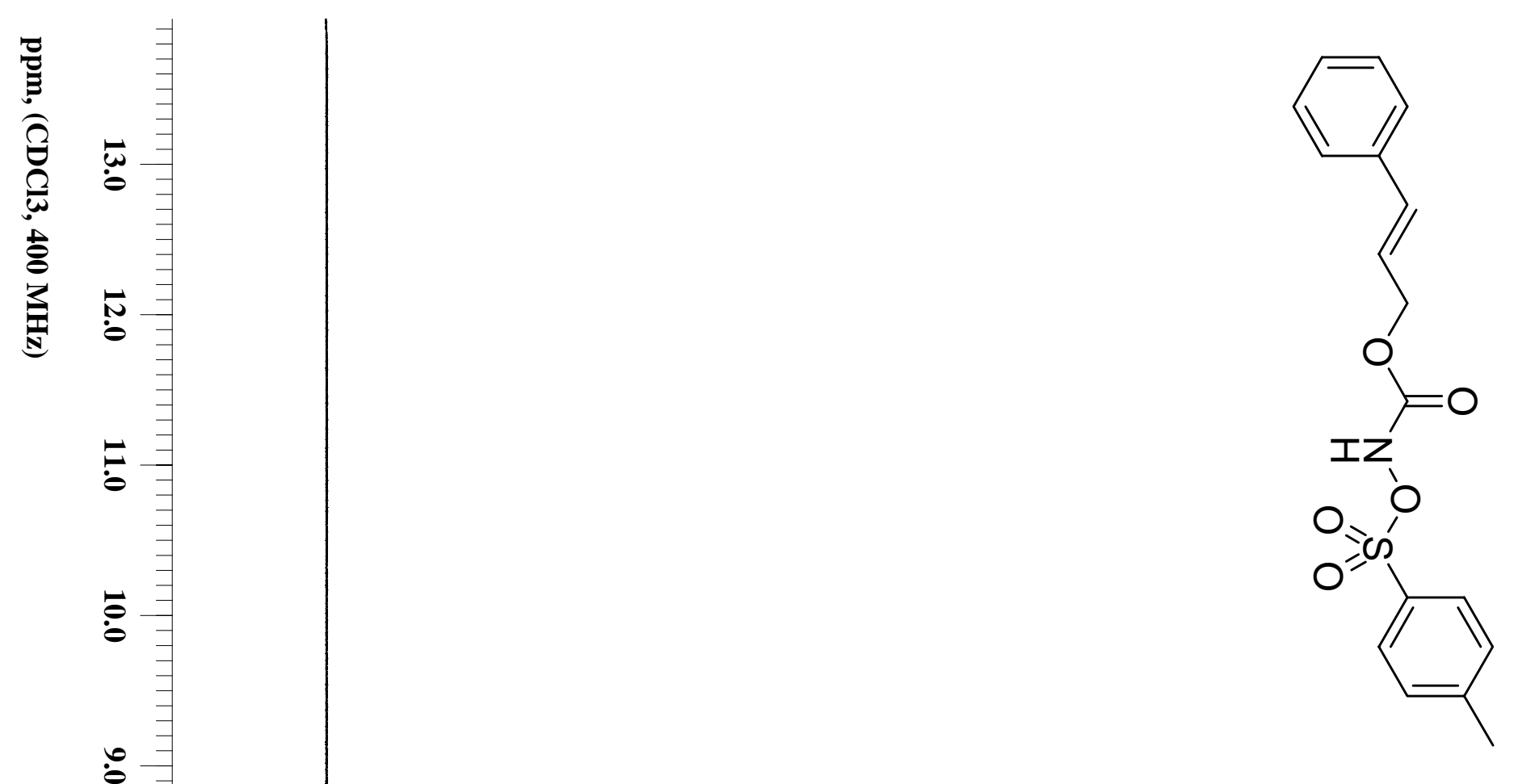




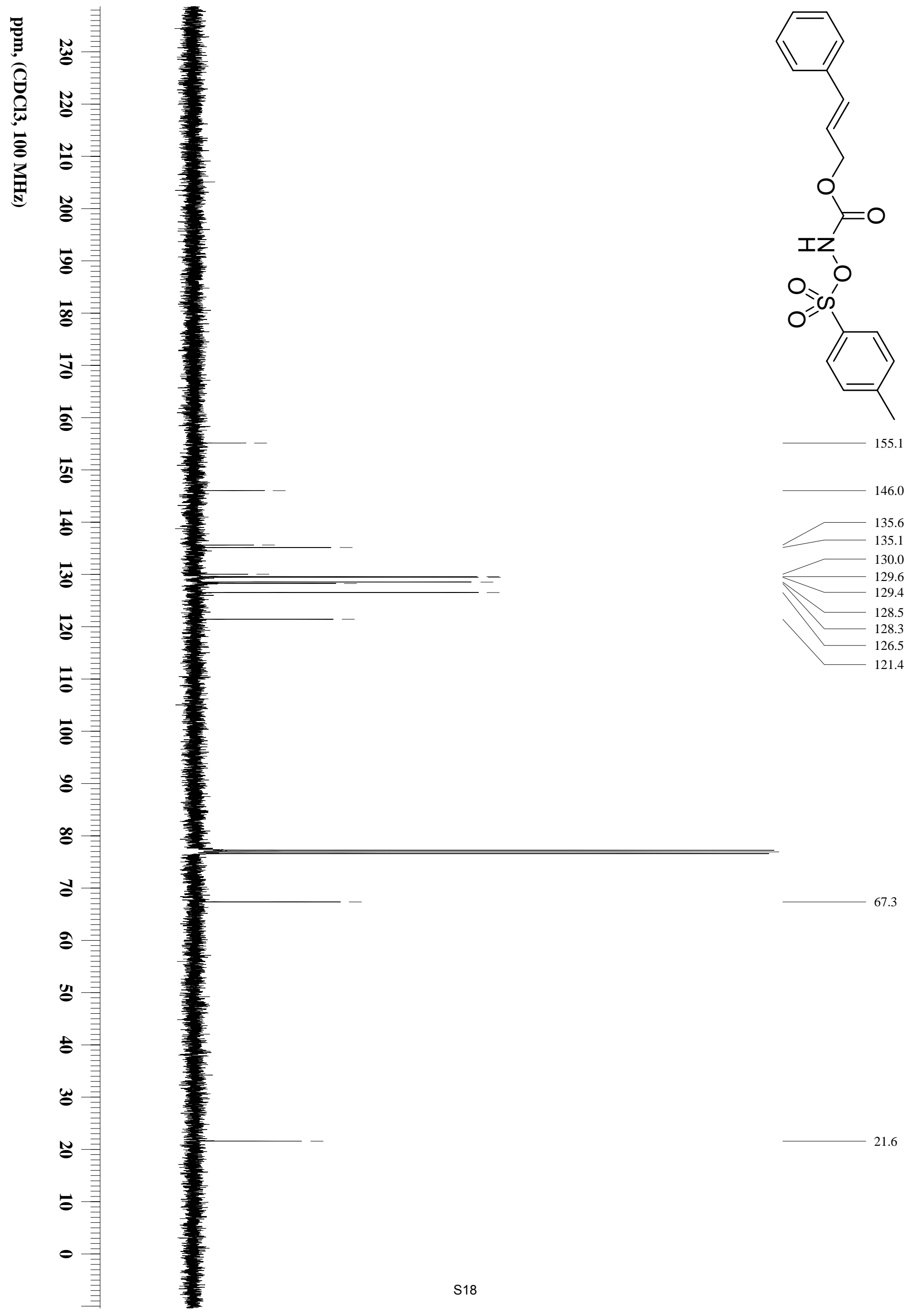




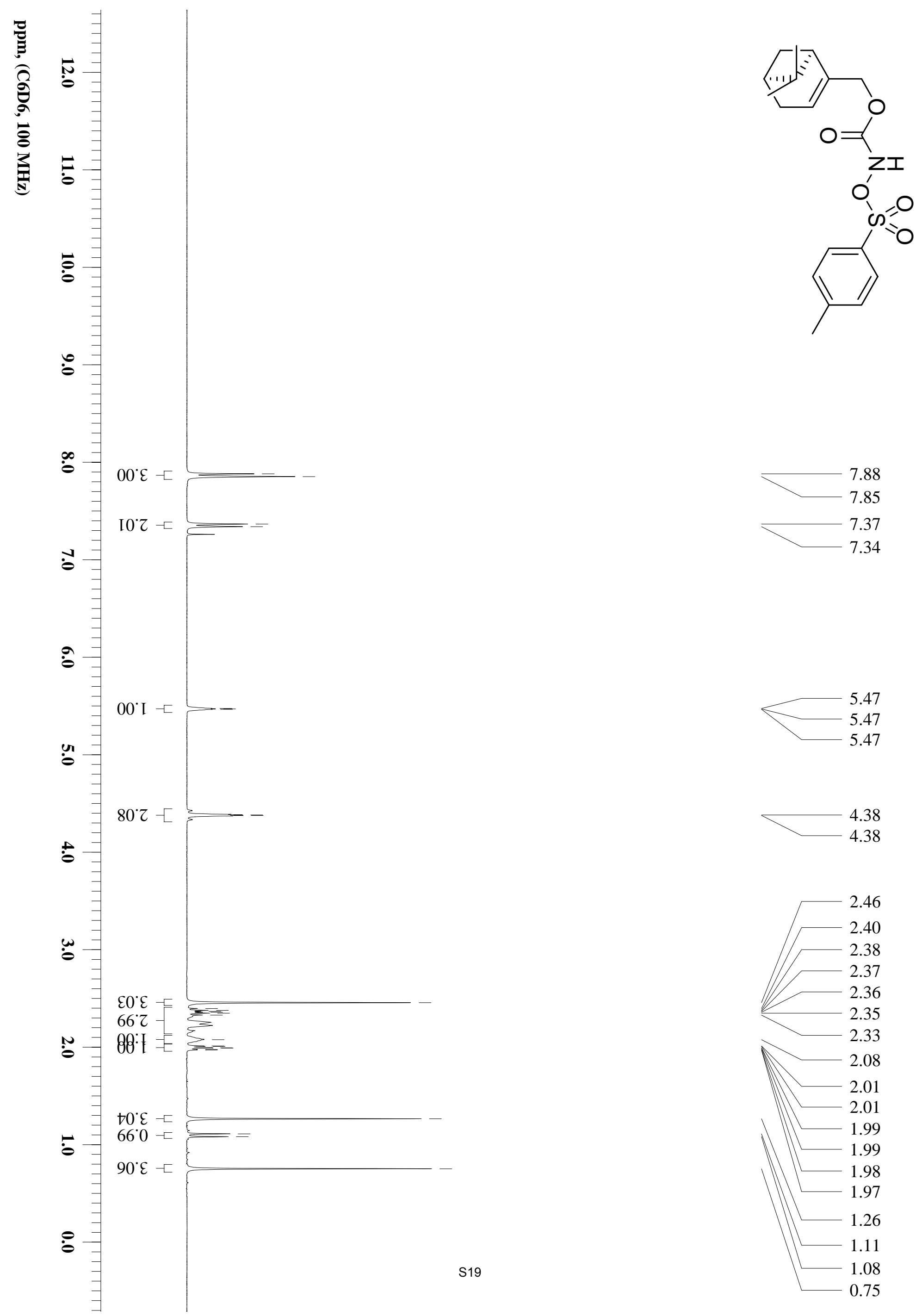




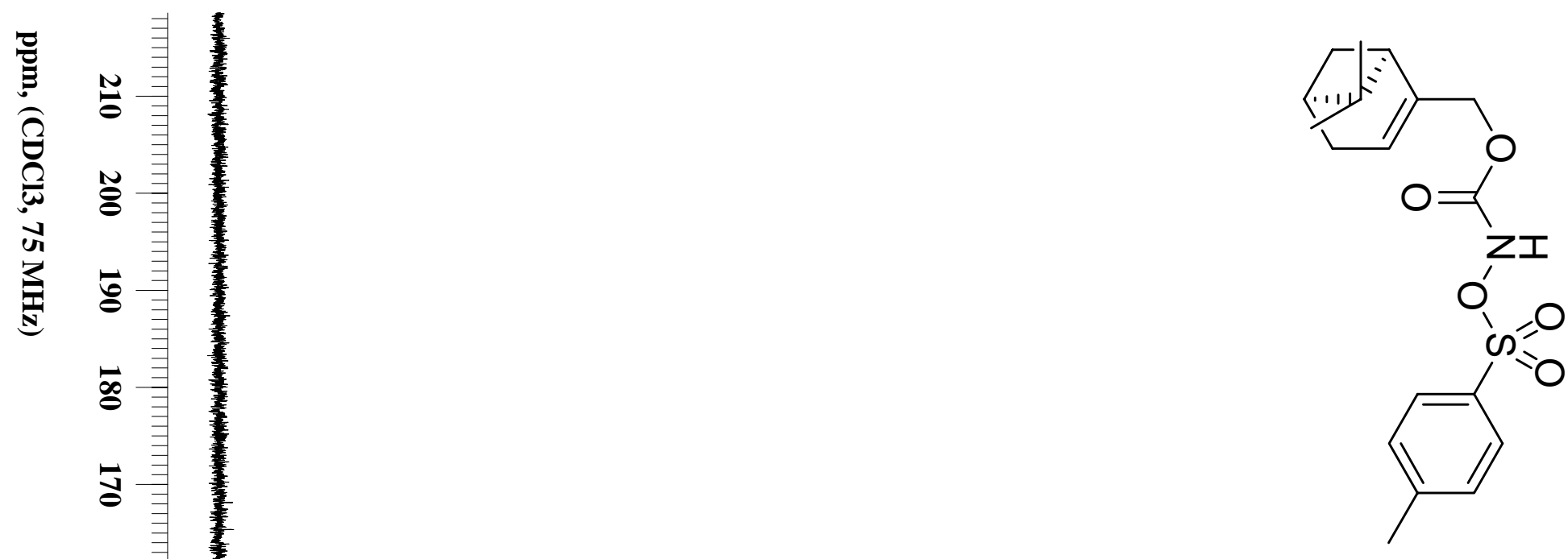

155.3

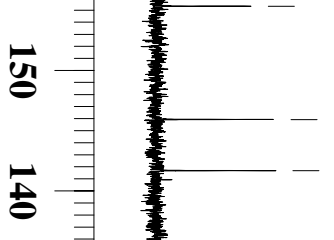

145.9

141.7

फ్

130.2

129.6

129.4

층

122.7

홍

s

8

ஜ

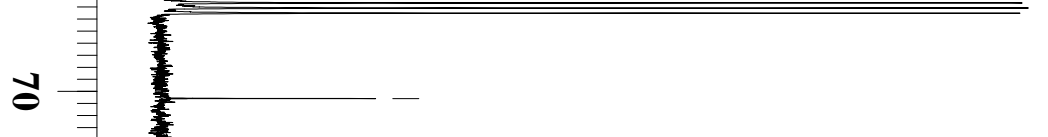

69.4

8

I

t

๗

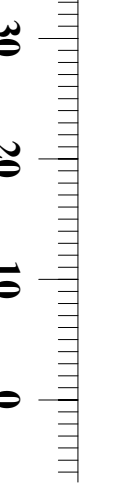




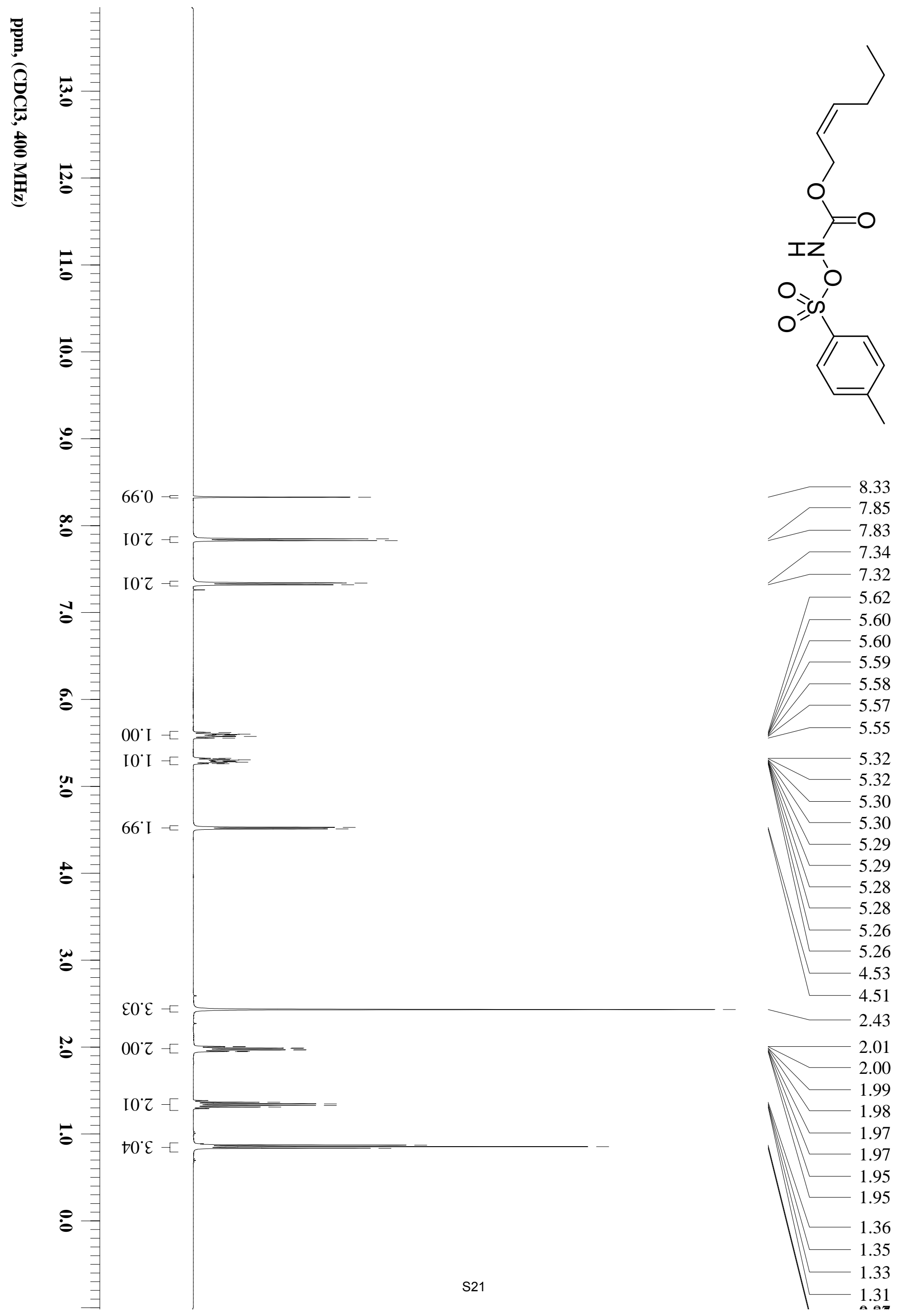




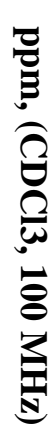

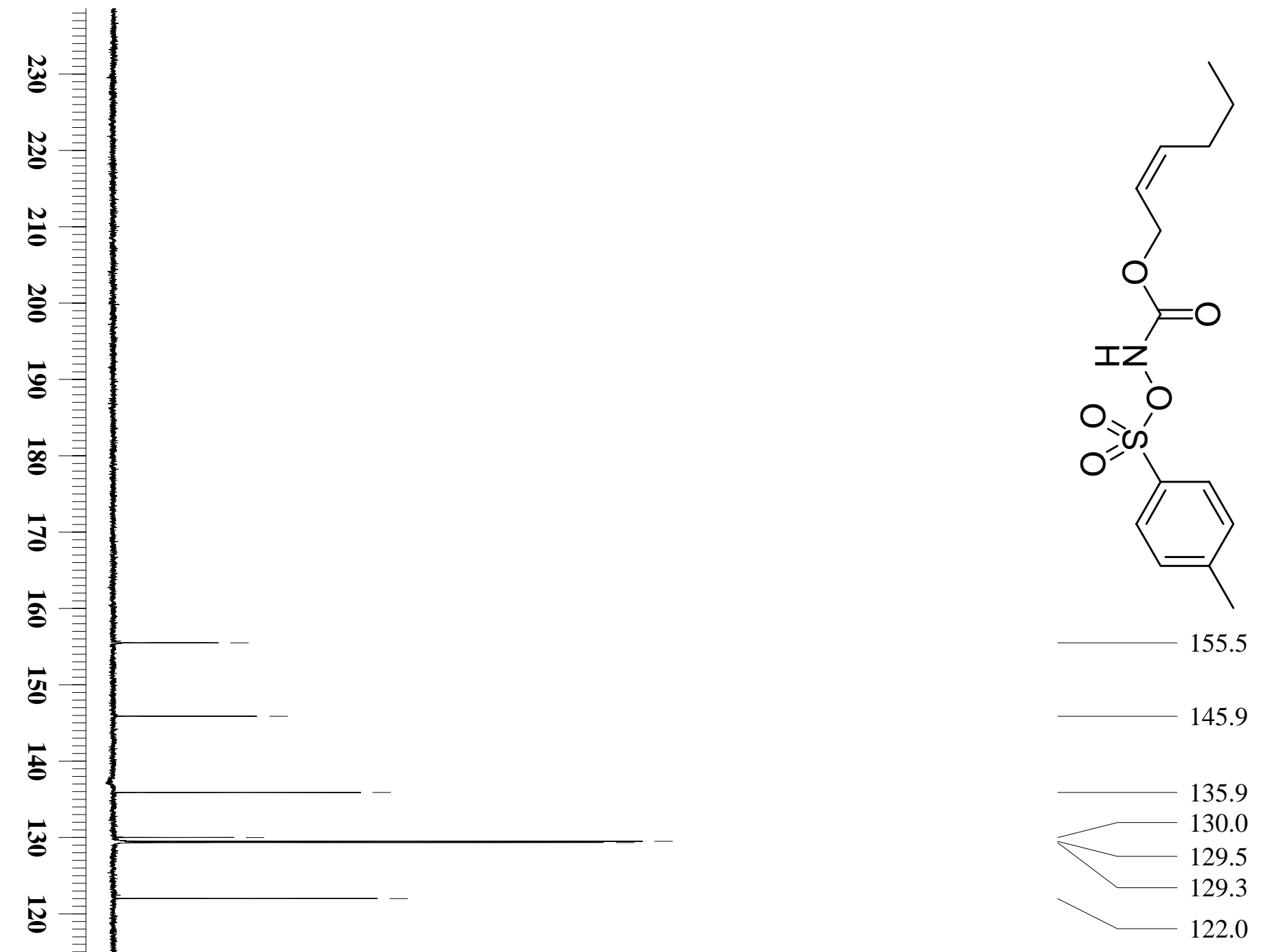

s

s

8

ஜ

d

o

당

8

w

$\approx$

b

$\circ$ 

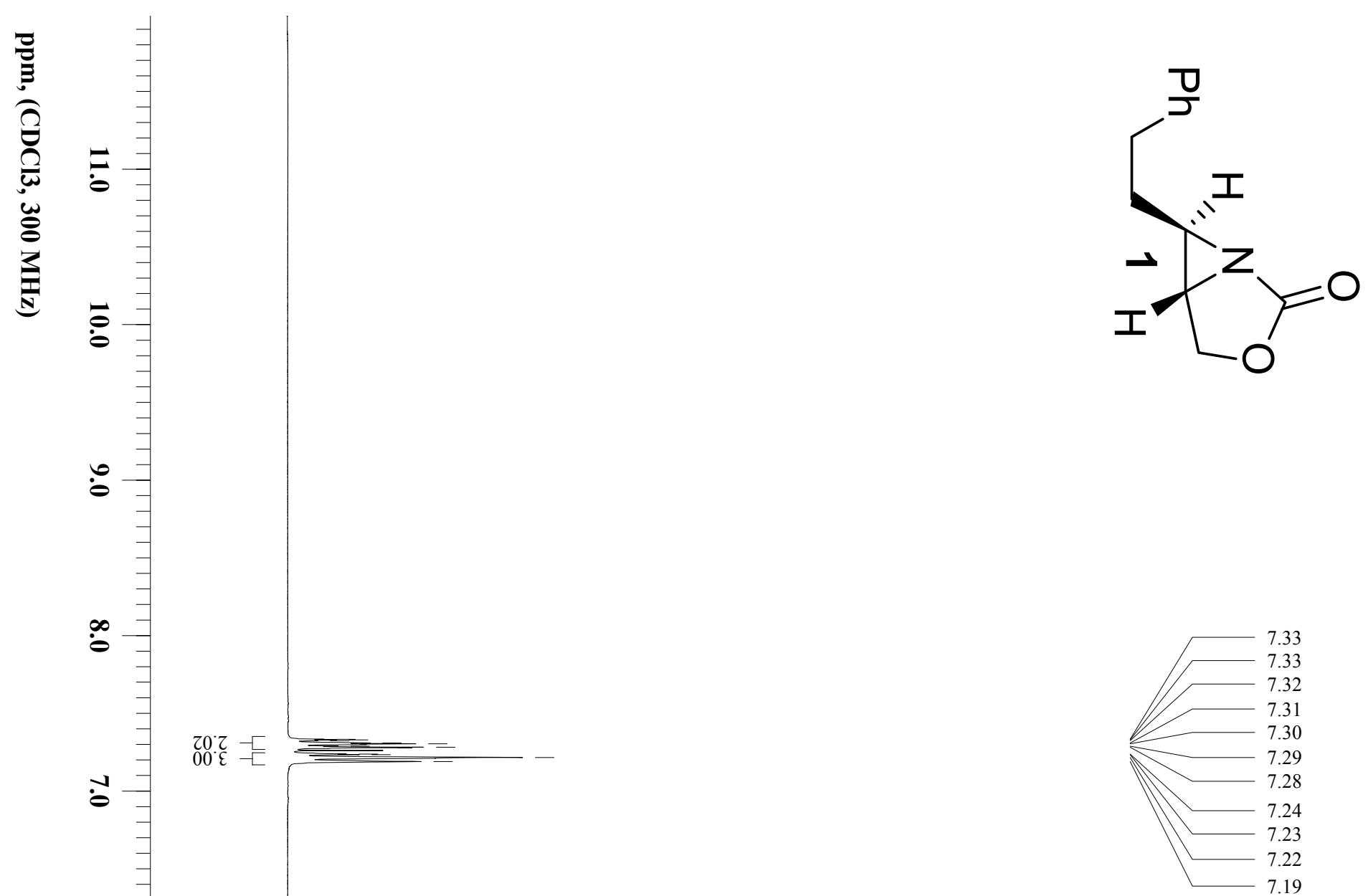

$\ddot{\theta}$

$\stackrel{d}{\circ}$
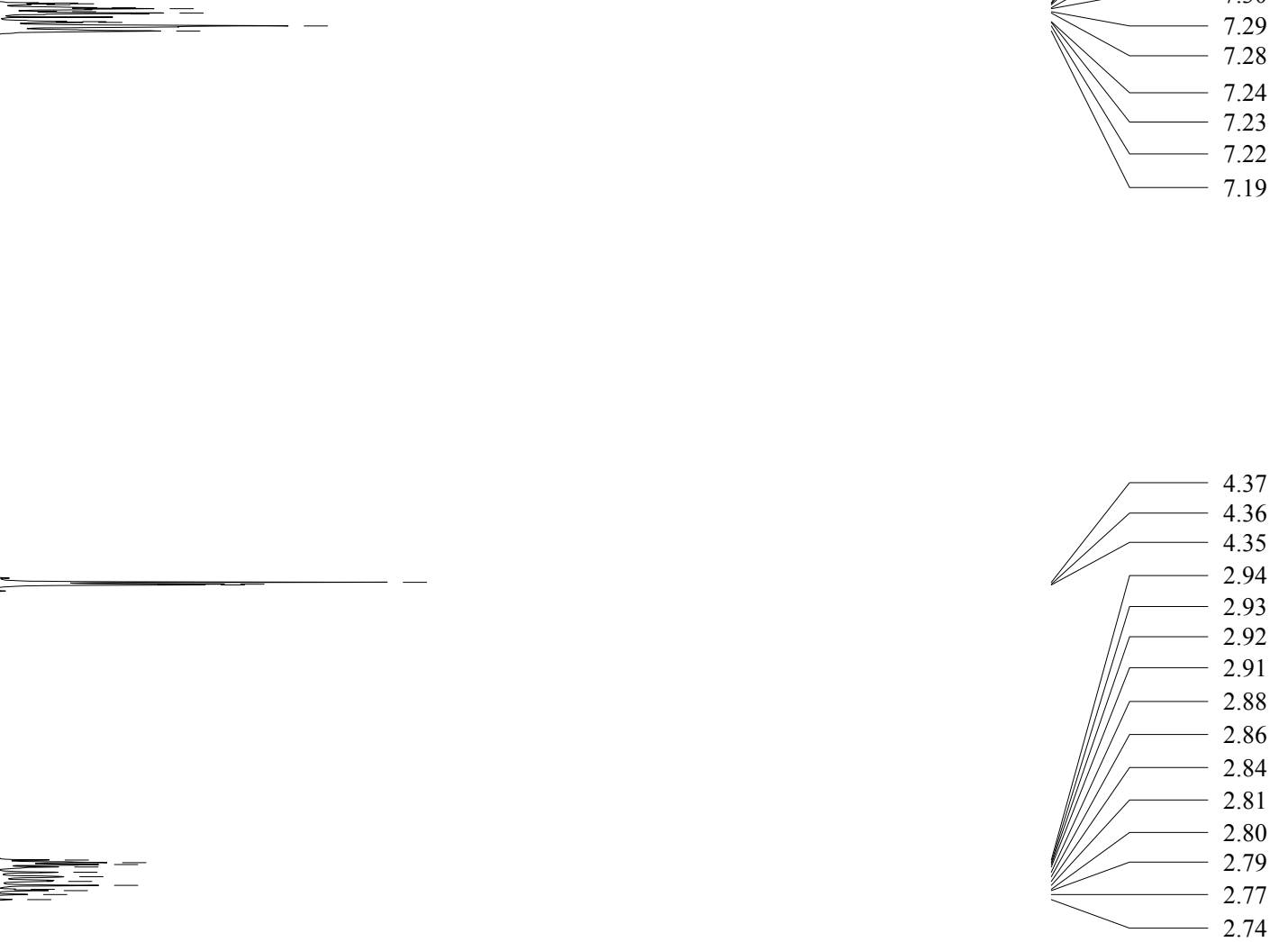

$\stackrel{+}{\dot{\theta}}$

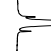

-

${ }_{96}^{\mathrm{S}} \mathrm{I}=\mathrm{I}=$

$660-\sqsubset=$

$\stackrel{N}{0}$

$007-$
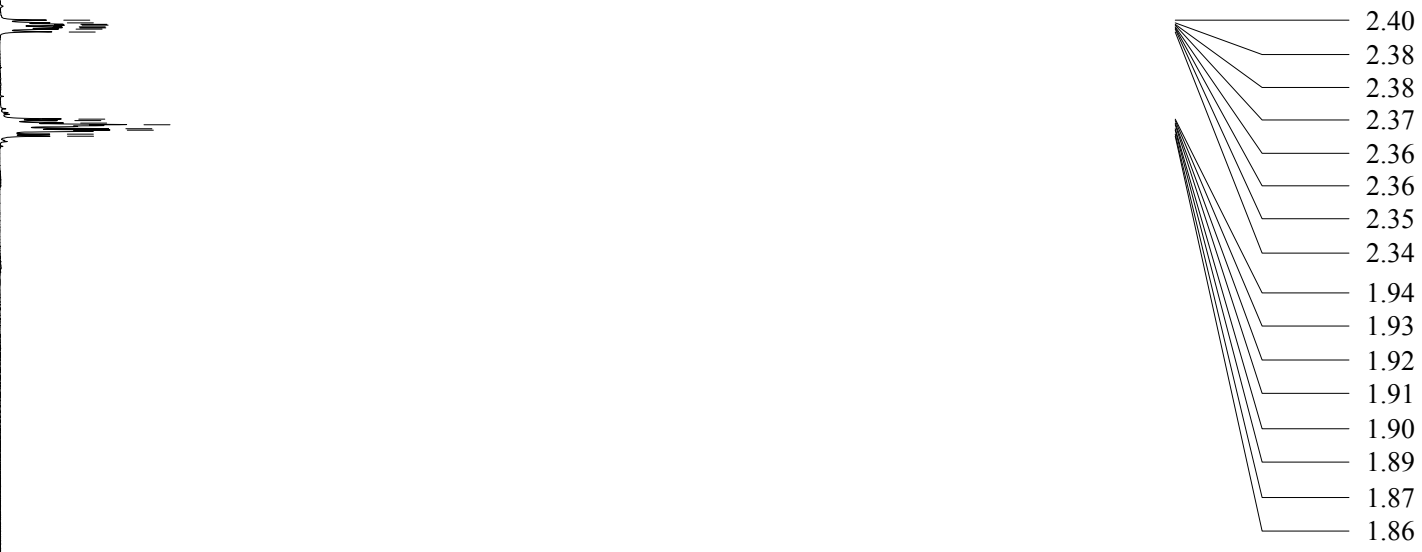

$\ddot{8}$
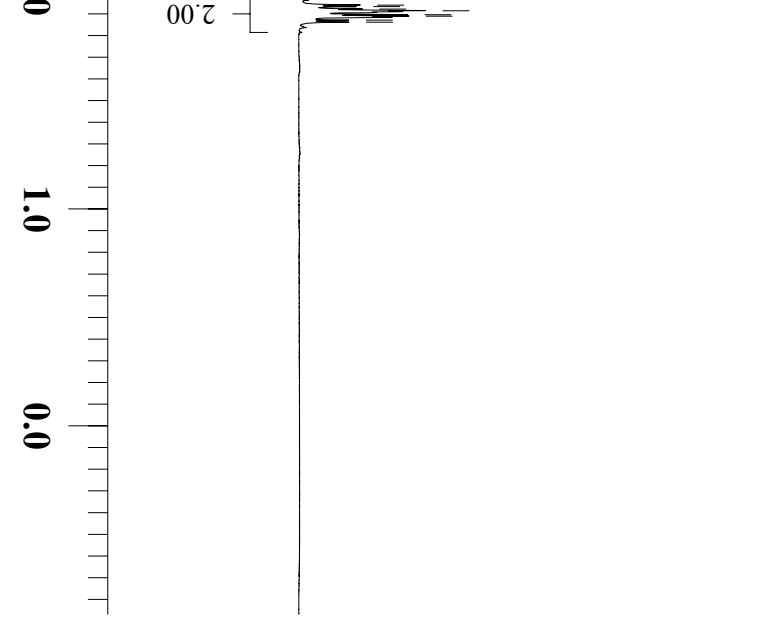


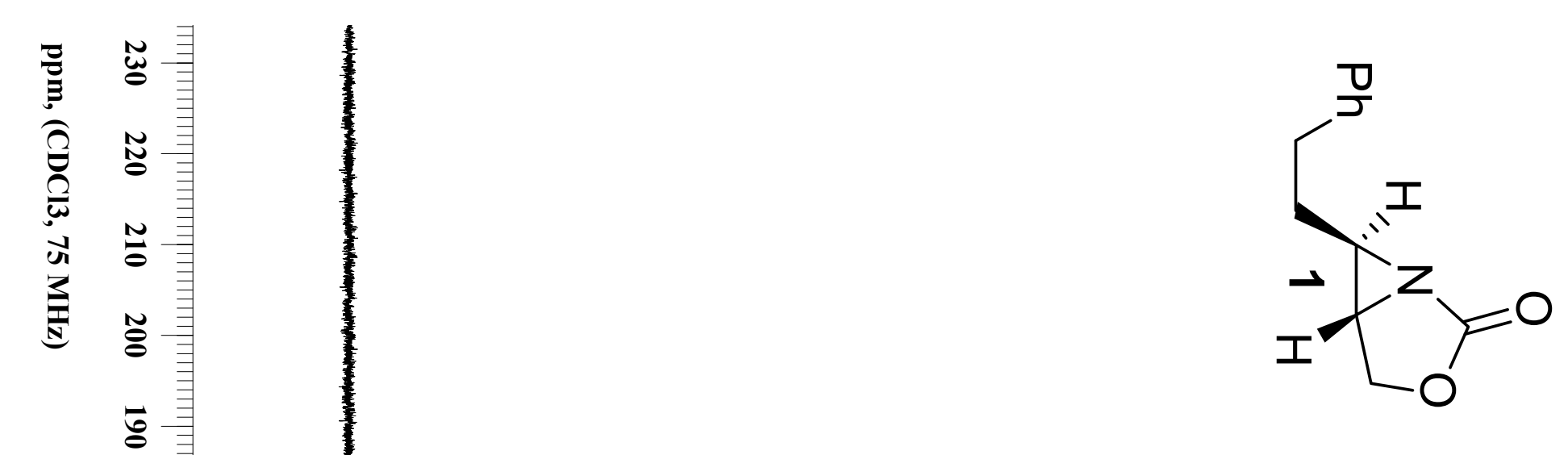

$\infty$

흐

166.9

ลㅇ

당

t)

140.2

๘)

กิ

ஏ

๕

๕

$\stackrel{\infty}{\circ}$
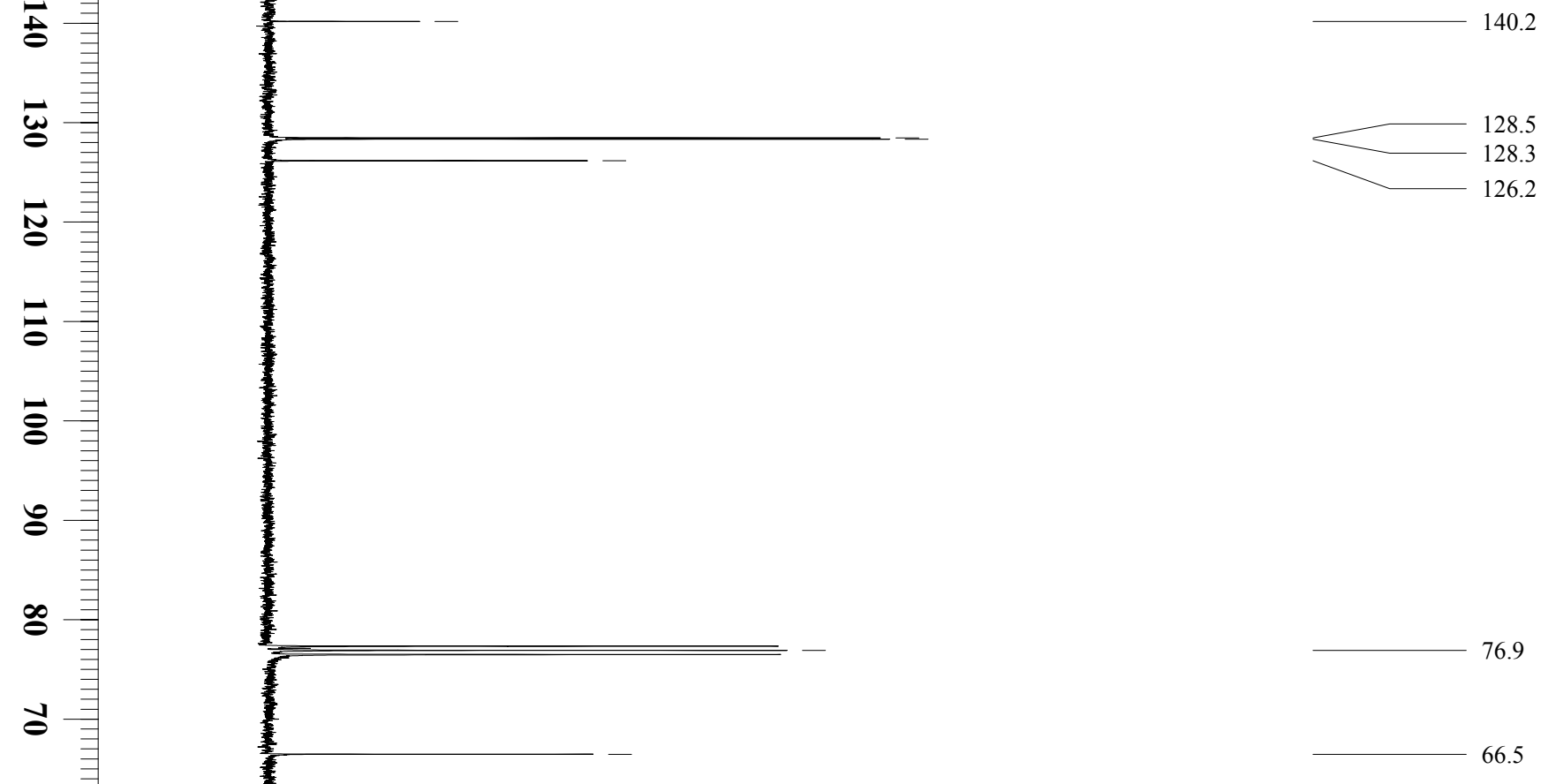

8

en

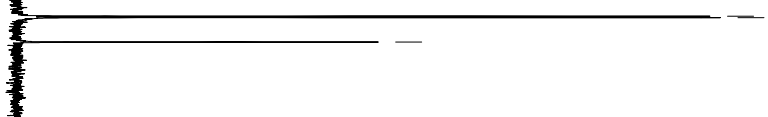

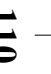

覀

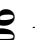

-

w

ำ

ㅎ

$\bullet$

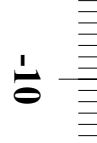


20ำ
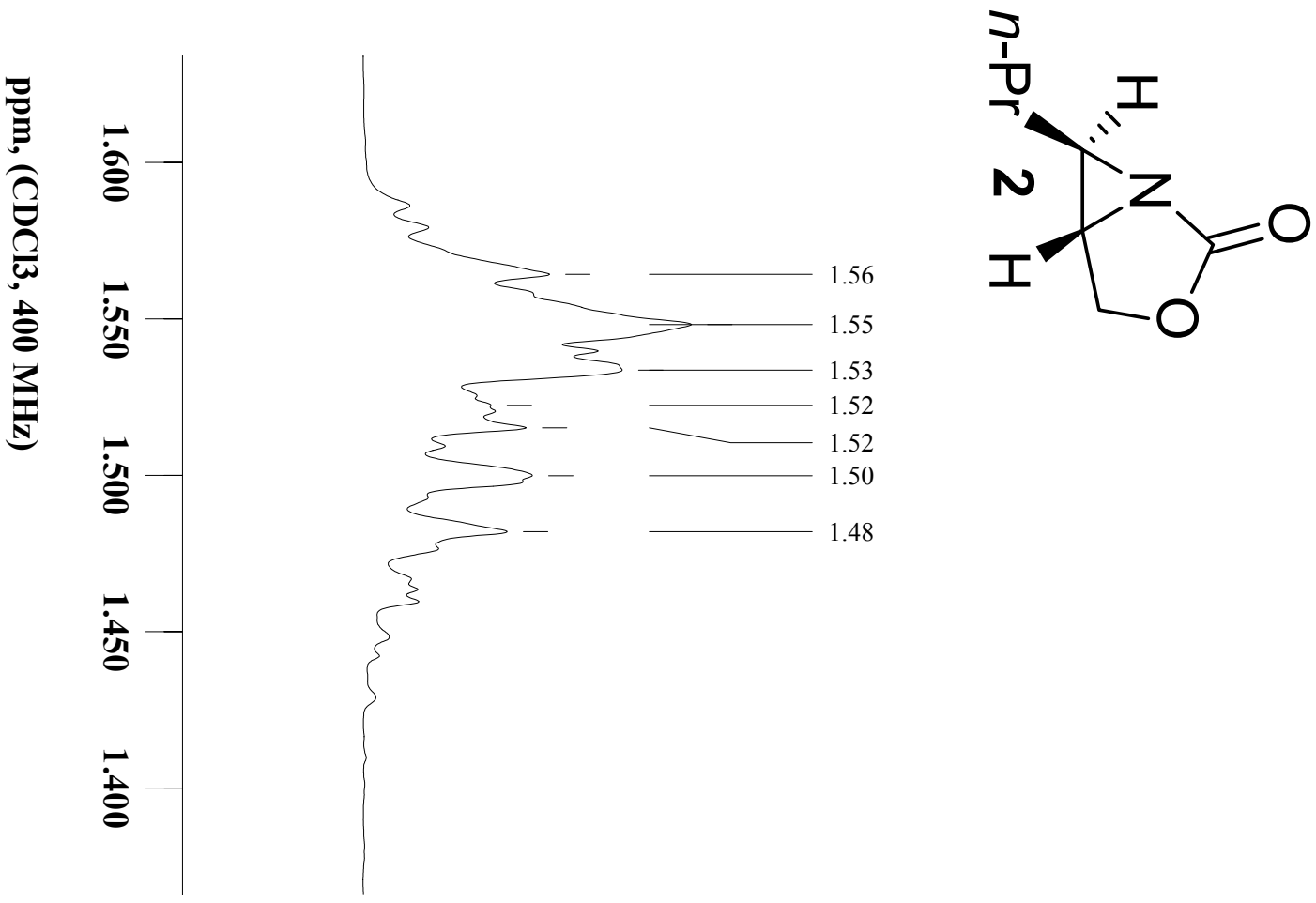

$\ddot{0}$

$\stackrel{6}{0}$

$\infty$

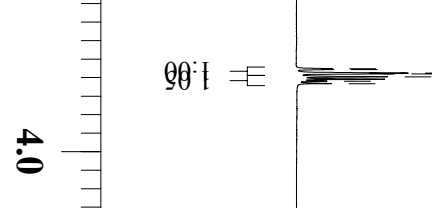

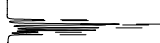

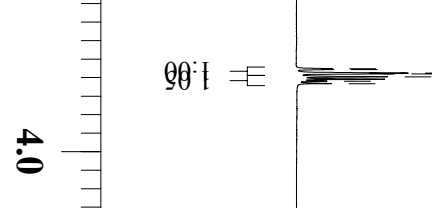

ச

$\stackrel{9}{0}$

v

u
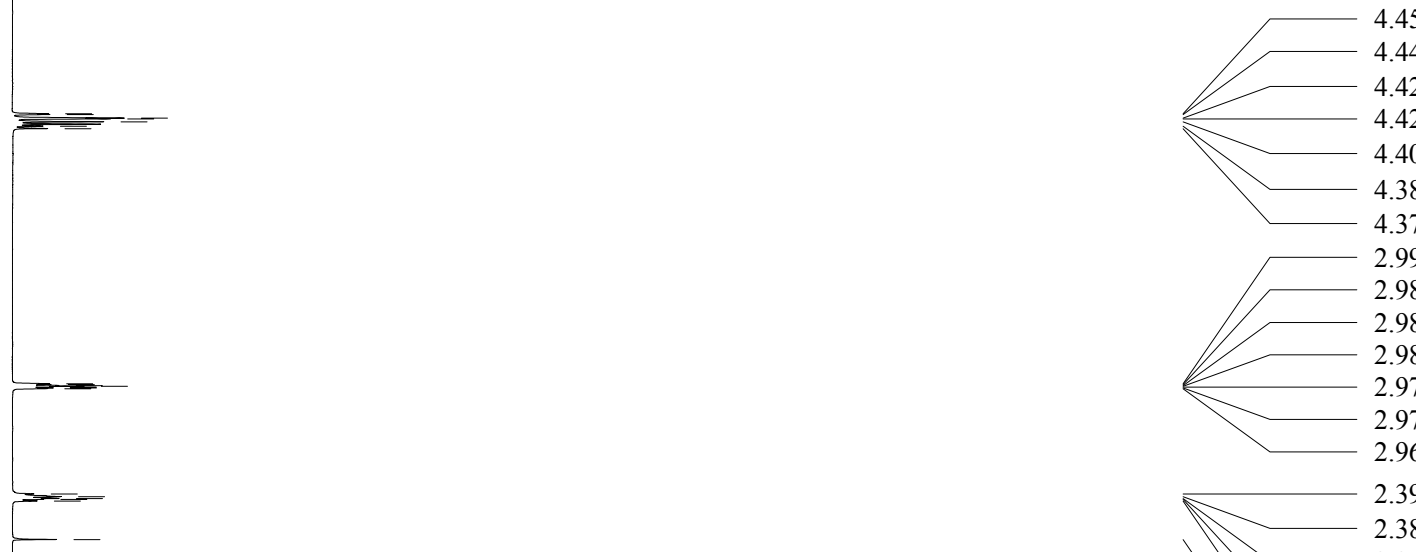

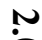

官
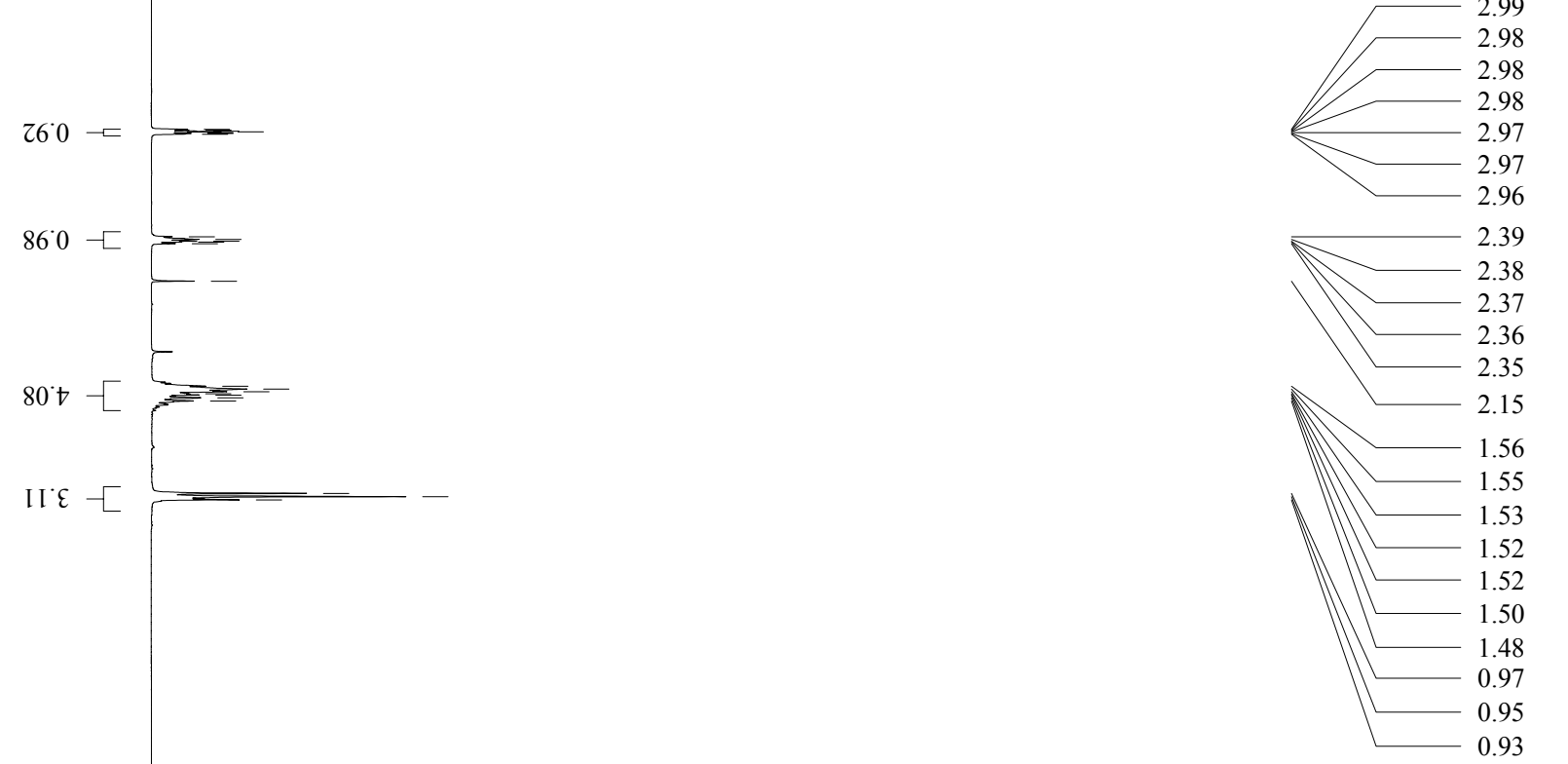
ชุำ

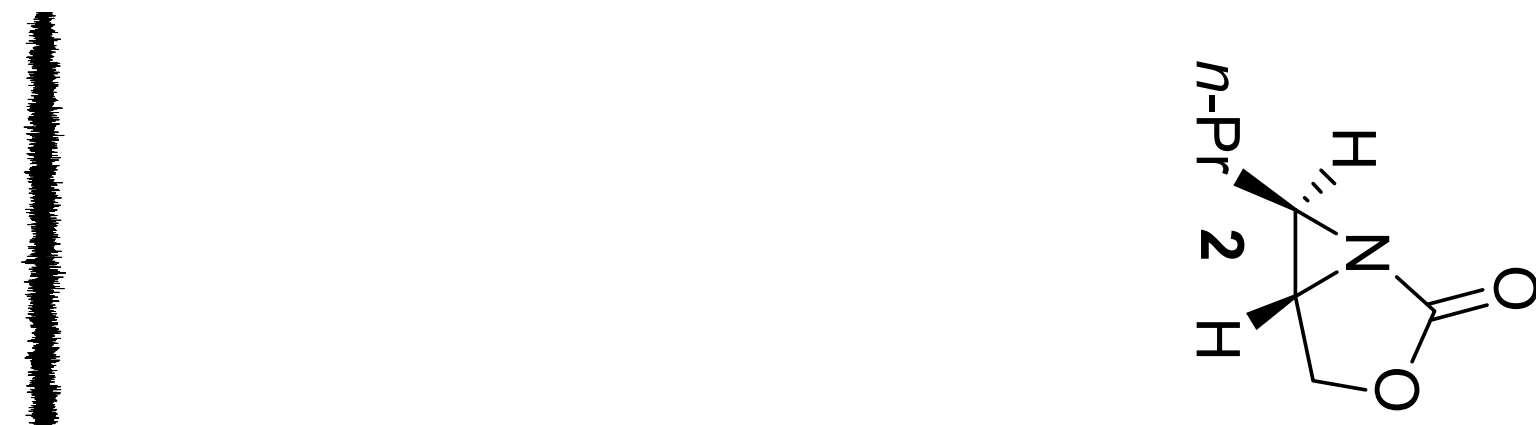

ธิ

$\overrightarrow{8}$

$\infty$

흐

167.1

5

항

E

ড్

ํํำ

$\Xi$

๕

๕

$\stackrel{8}{\circ}$

ฮิ

8

당

$t$

щٌ

ำ

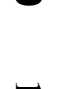

$\overline{0}$ 

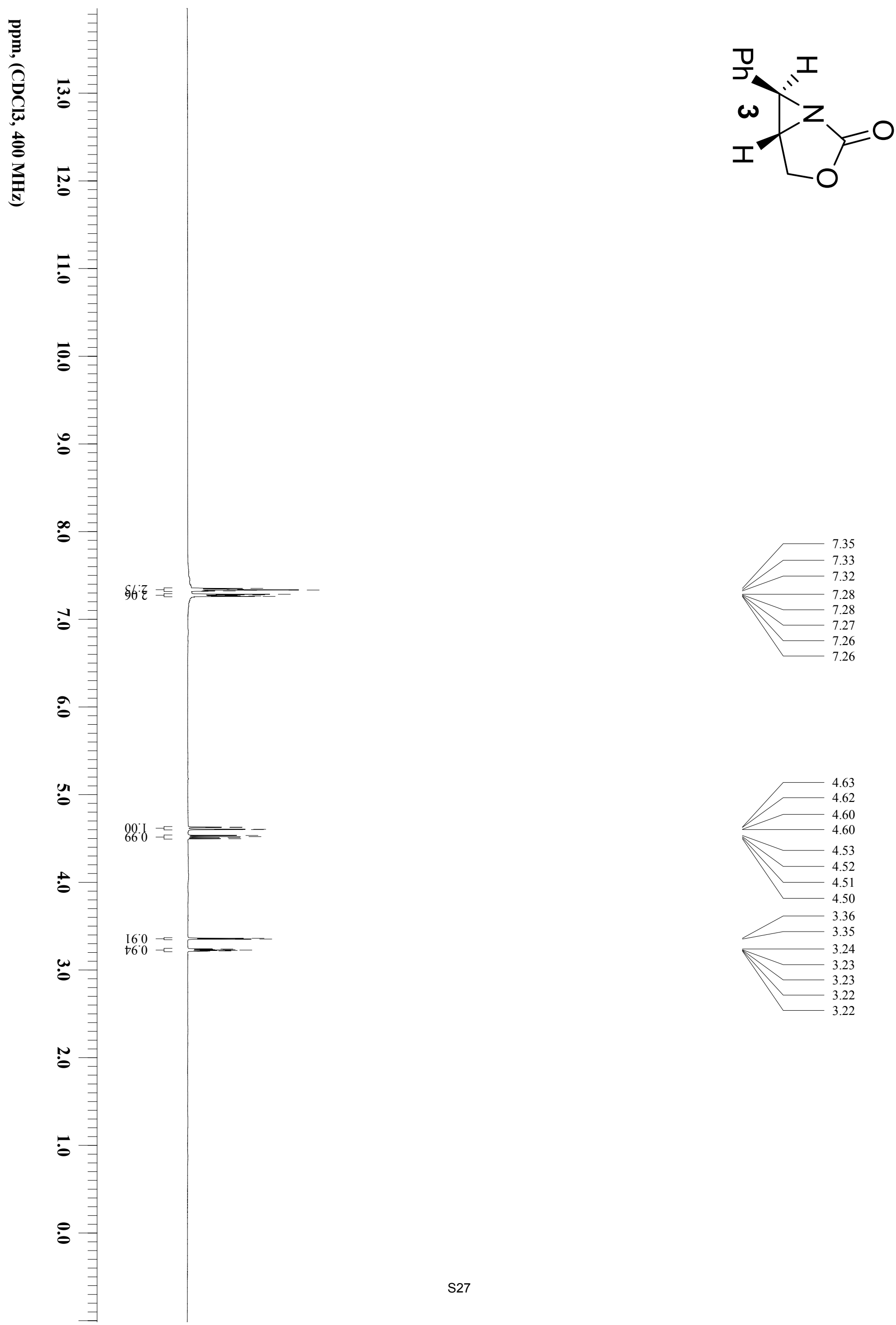

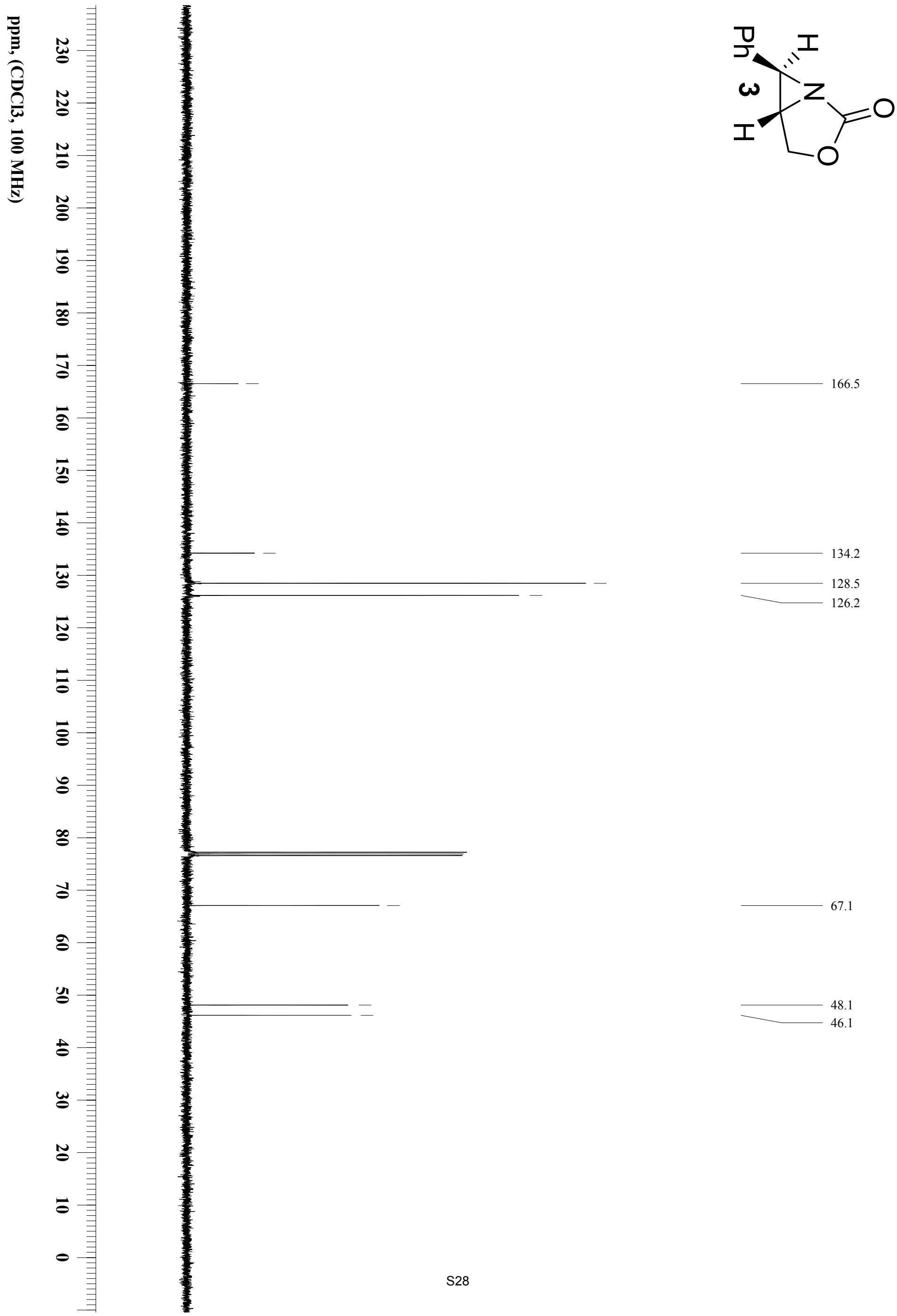

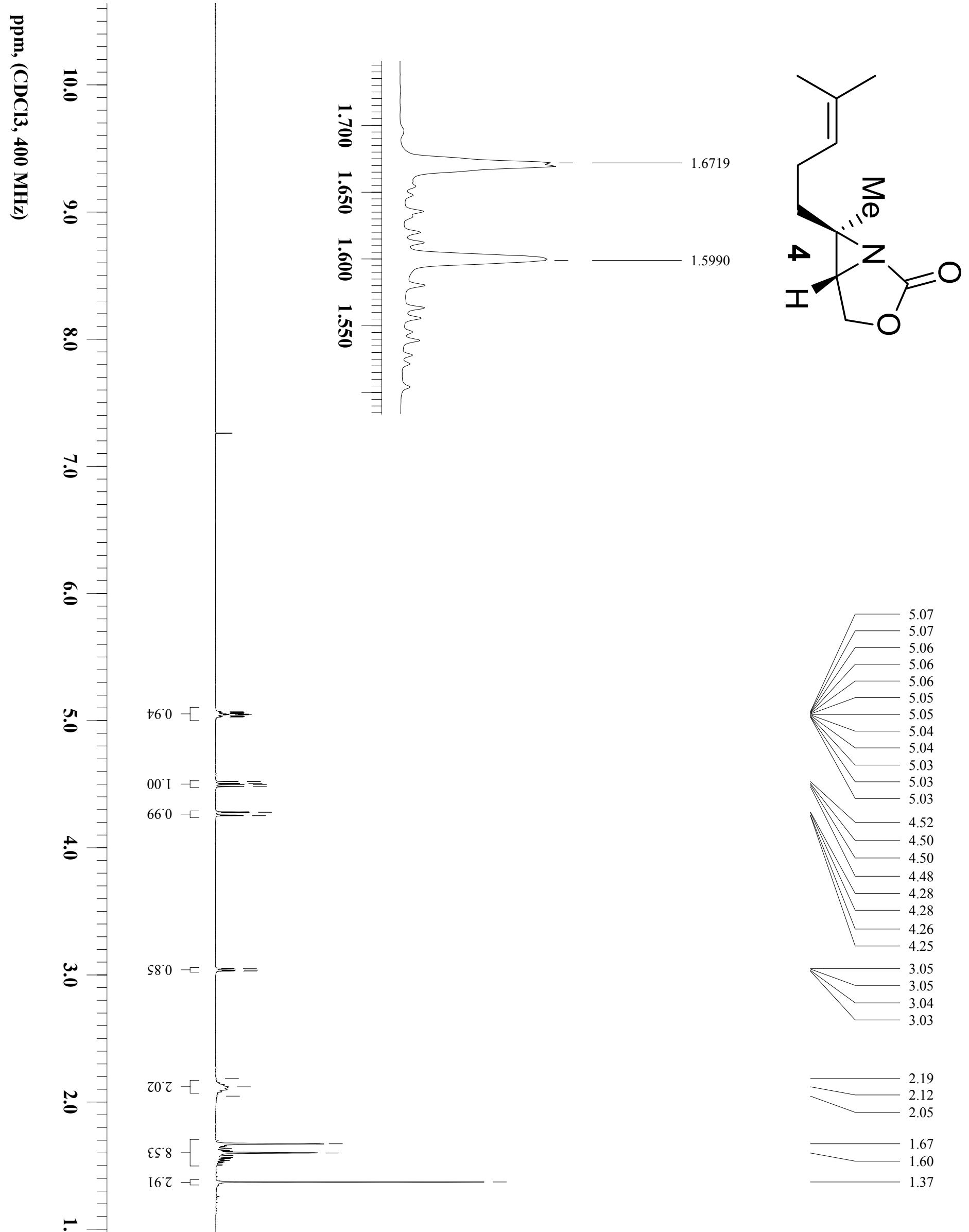

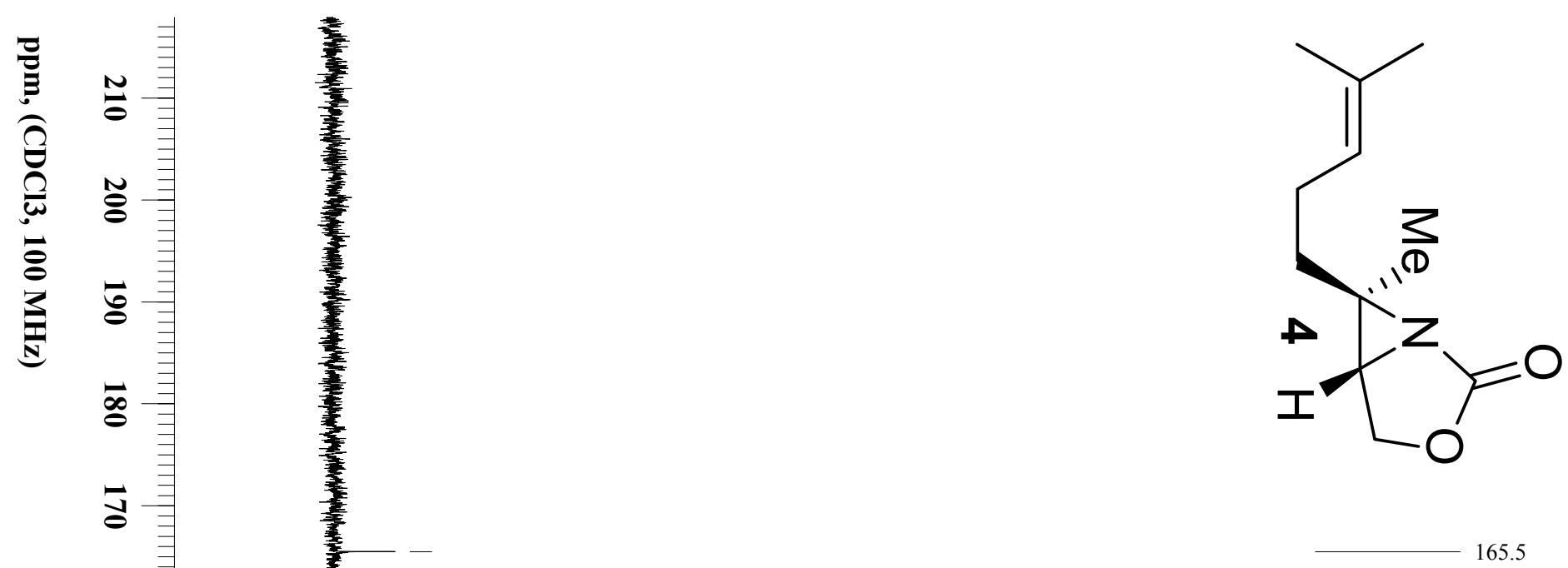

132.6

$\bar{\varpi}$

항

E

్ㅡㅇ

흥

\&

8

$\stackrel{\circ}{\circ}$

d

8

잉

$\pm$

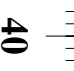

\&

ก

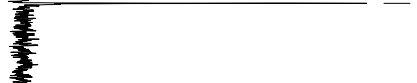

122.6

64.5

50.9

48.8

38.5

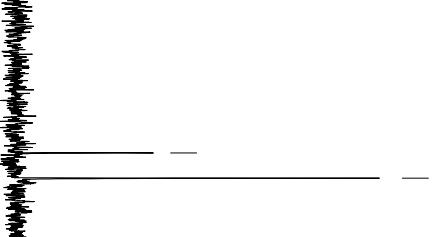

\&

$\circ$ 


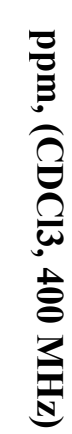

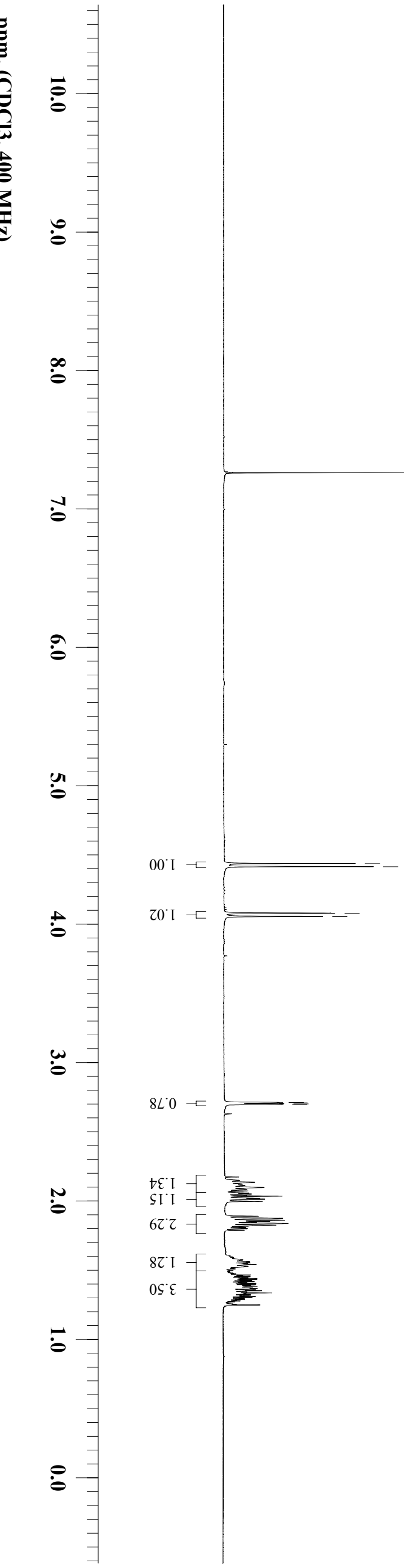

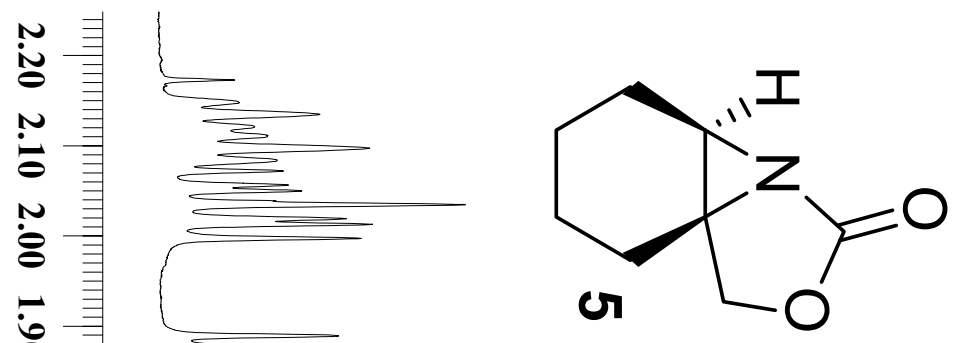
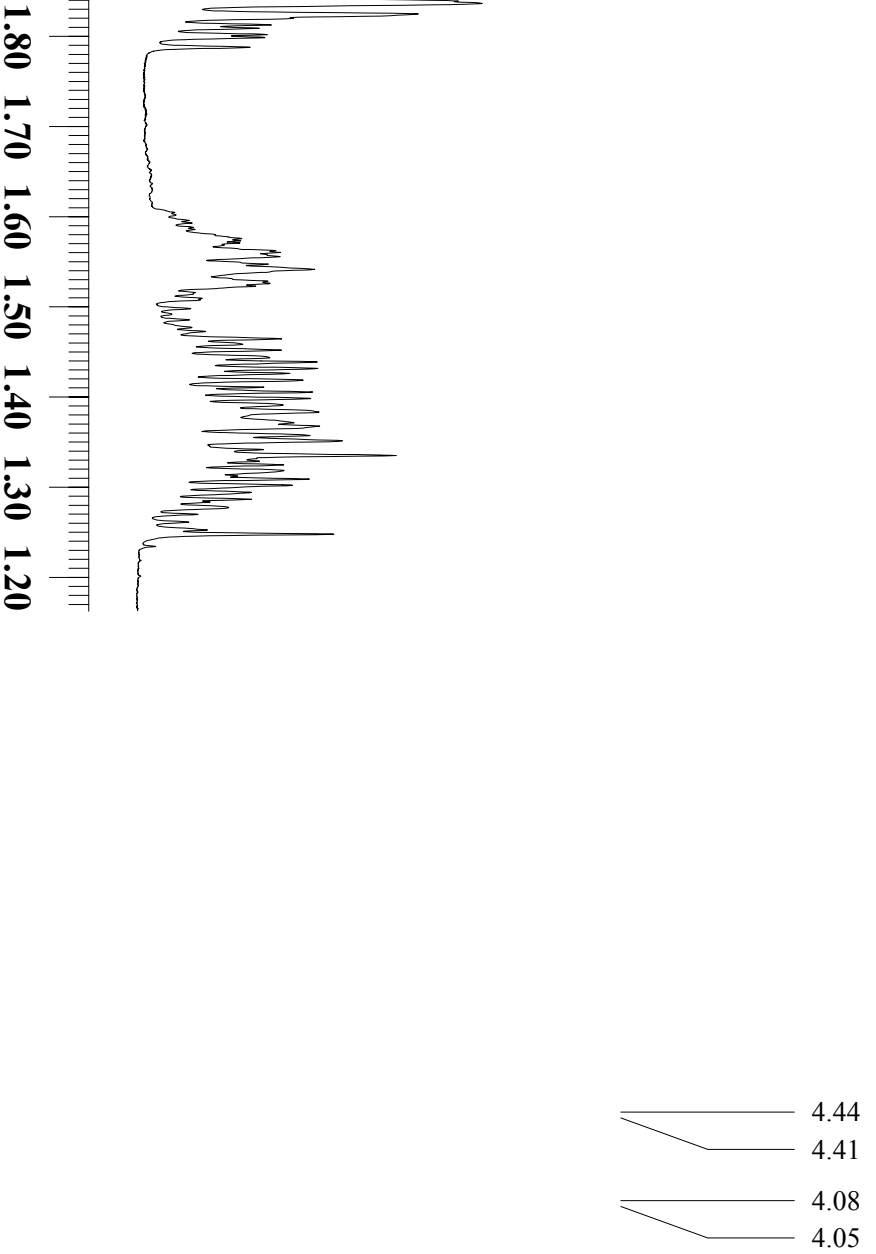

2.71

2.71

2.70 

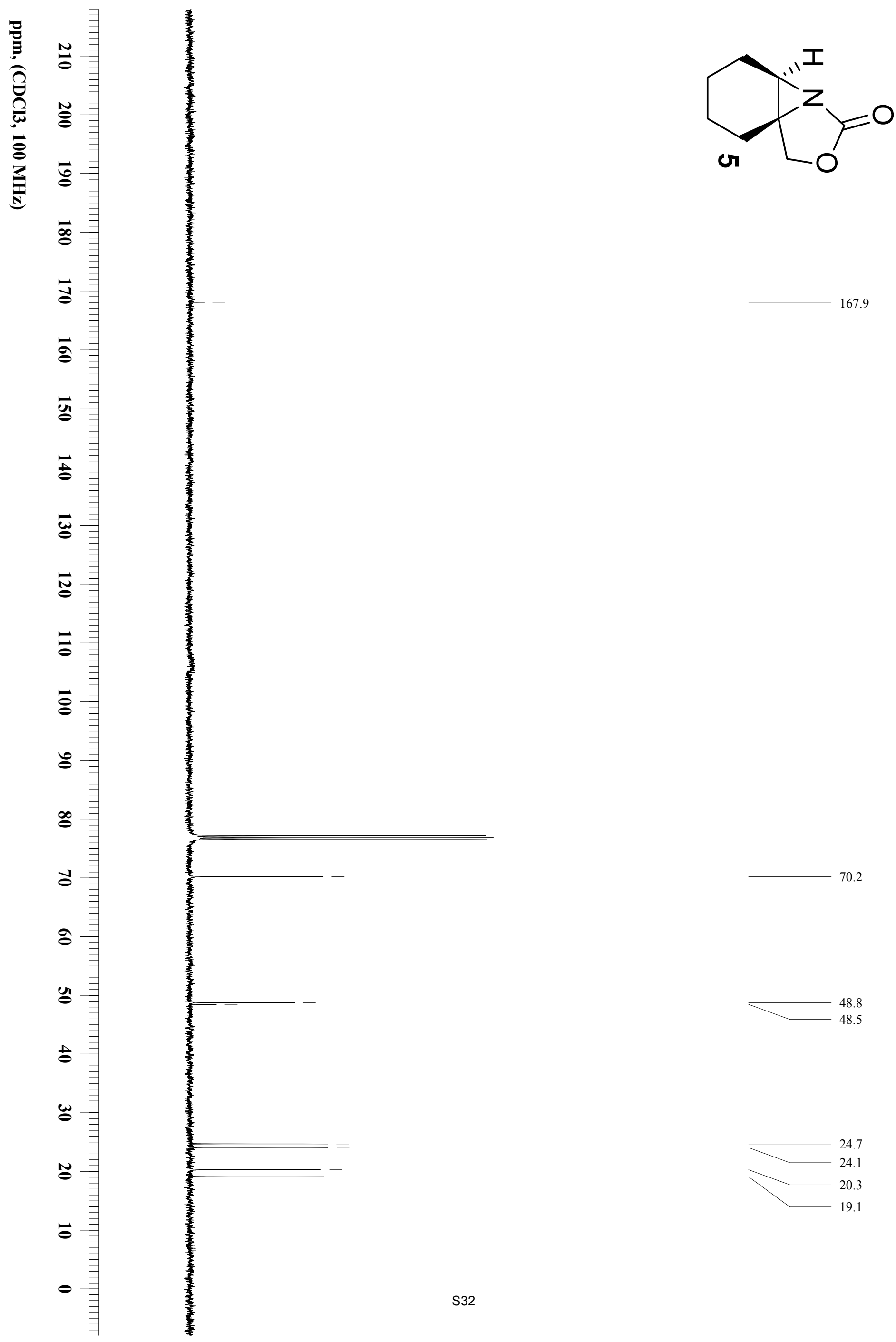

167.9

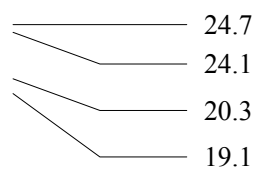



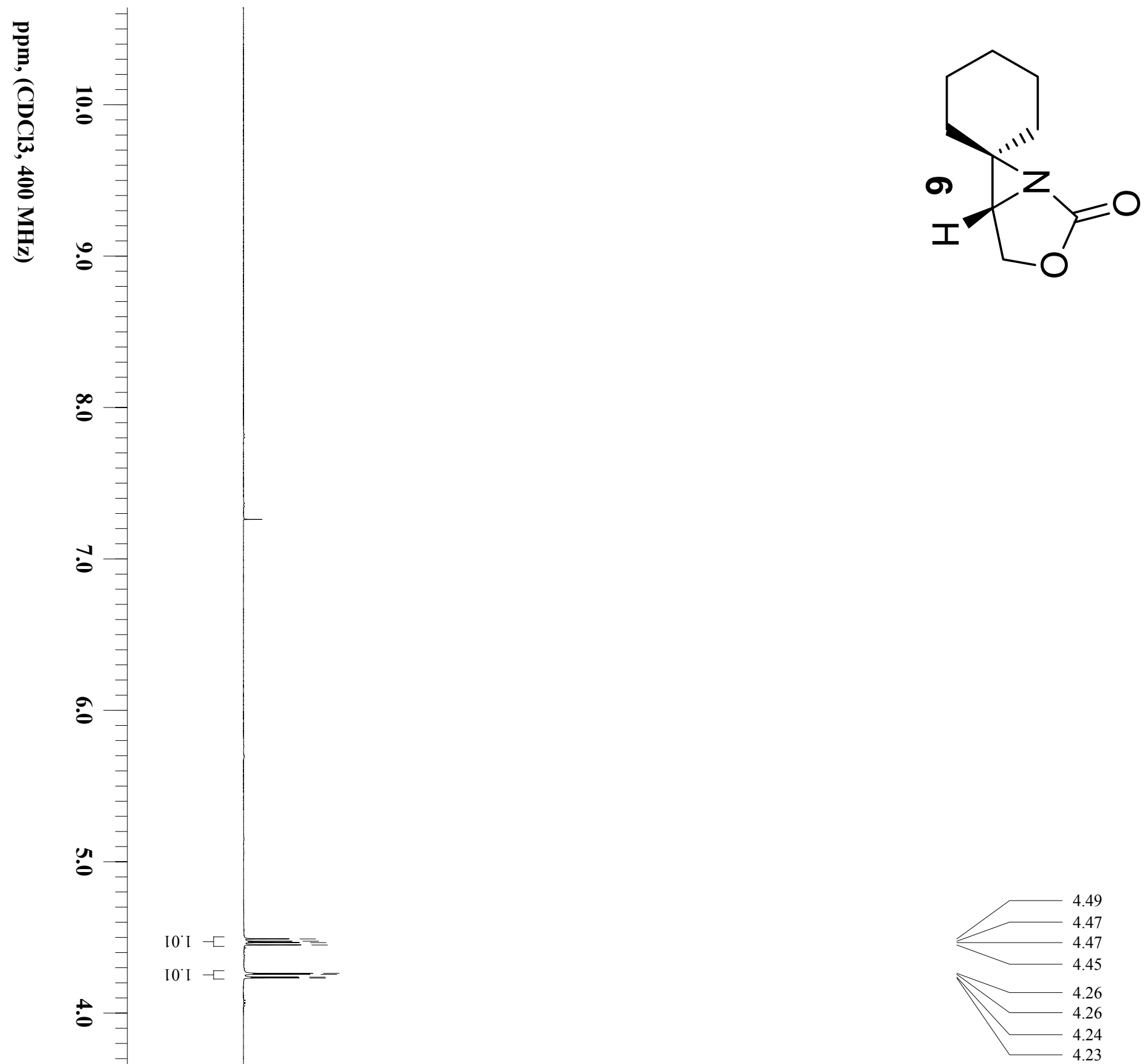


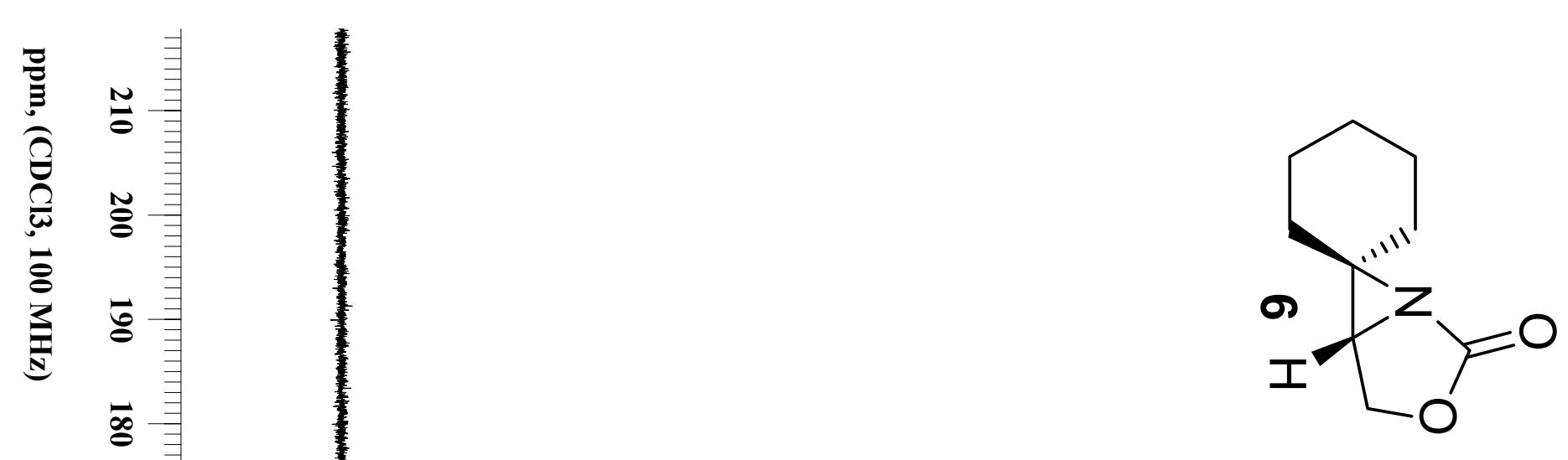

165.4

8

$\overline{\mathrm{g}}$

ธั

$\bar{\sigma}$

ธิ

8

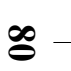

ฮ

8

背

$t$

$\stackrel{\omega}{\circ}$

ก

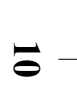

0 

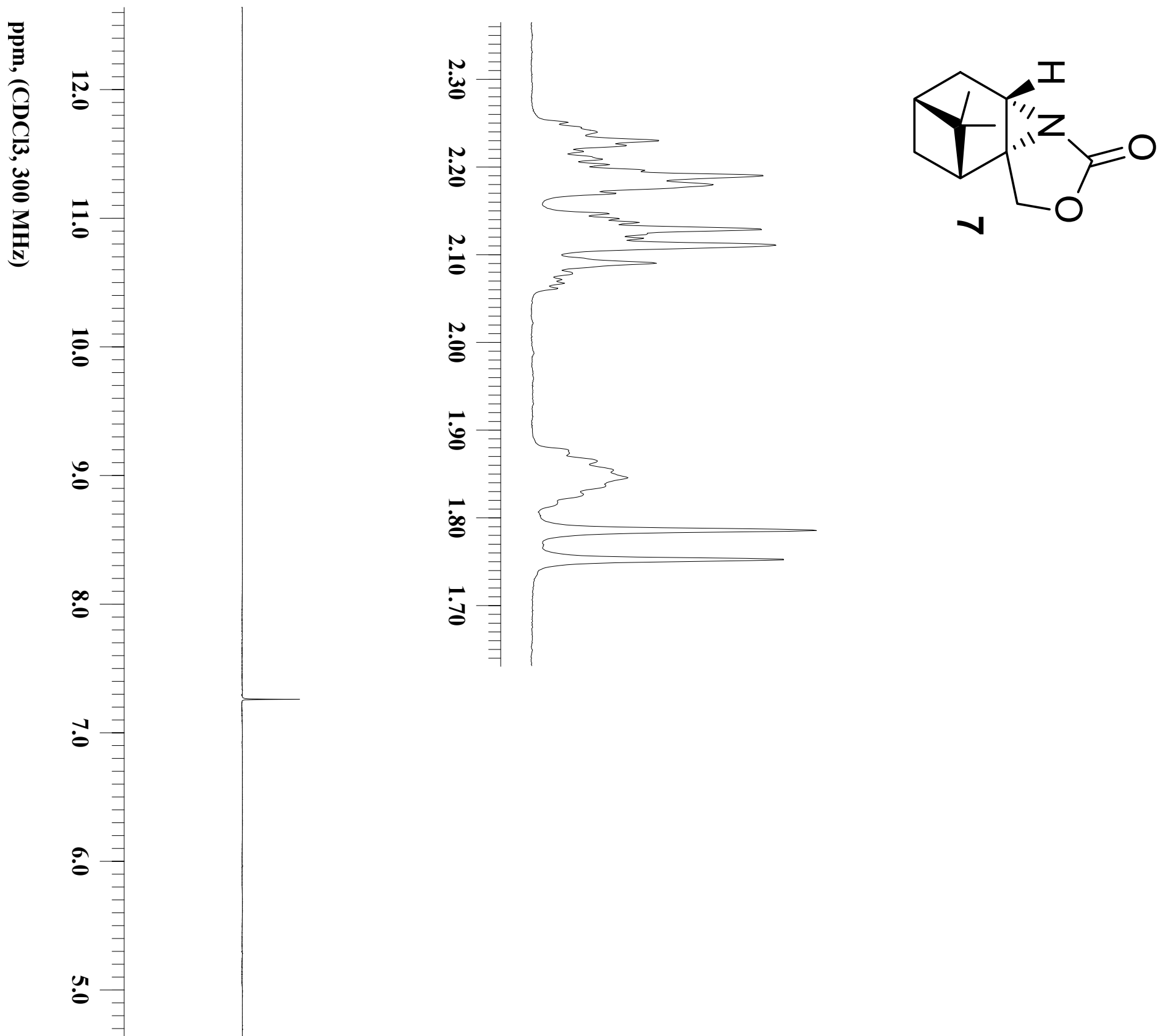

$00^{\circ} \mathrm{C} \sqsubset$

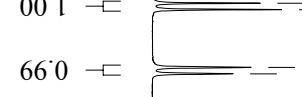

$\stackrel{\oplus}{\circ}$
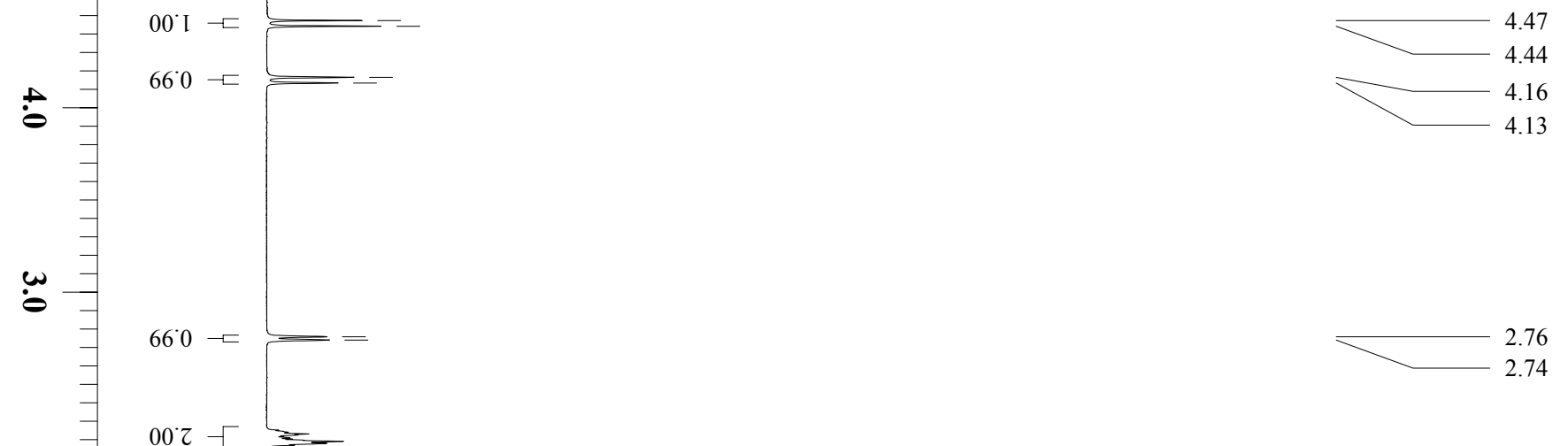

$\stackrel{N}{\circ}$

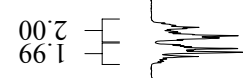

$66: 0=$

$668-2 \sum-$

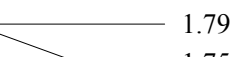

1.75

$\dot{\theta}$

$\tau 0^{\circ} \varepsilon-\sqsubset$

เ $0^{\circ} \mathcal{E} \sqsubset$ 


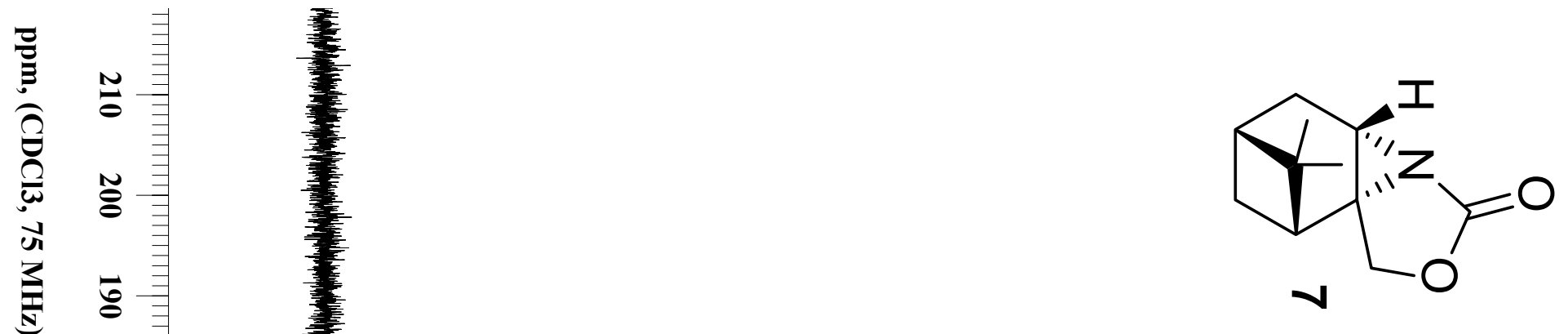

g

$\bar{\Xi}$

166.5

g

깅

E

$\bar{g}$

ลิ

후

$\overline{8}$

8

$\stackrel{8}{\circ}$

$\checkmark$

。

y

e

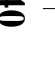

๘

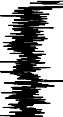

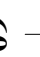

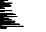



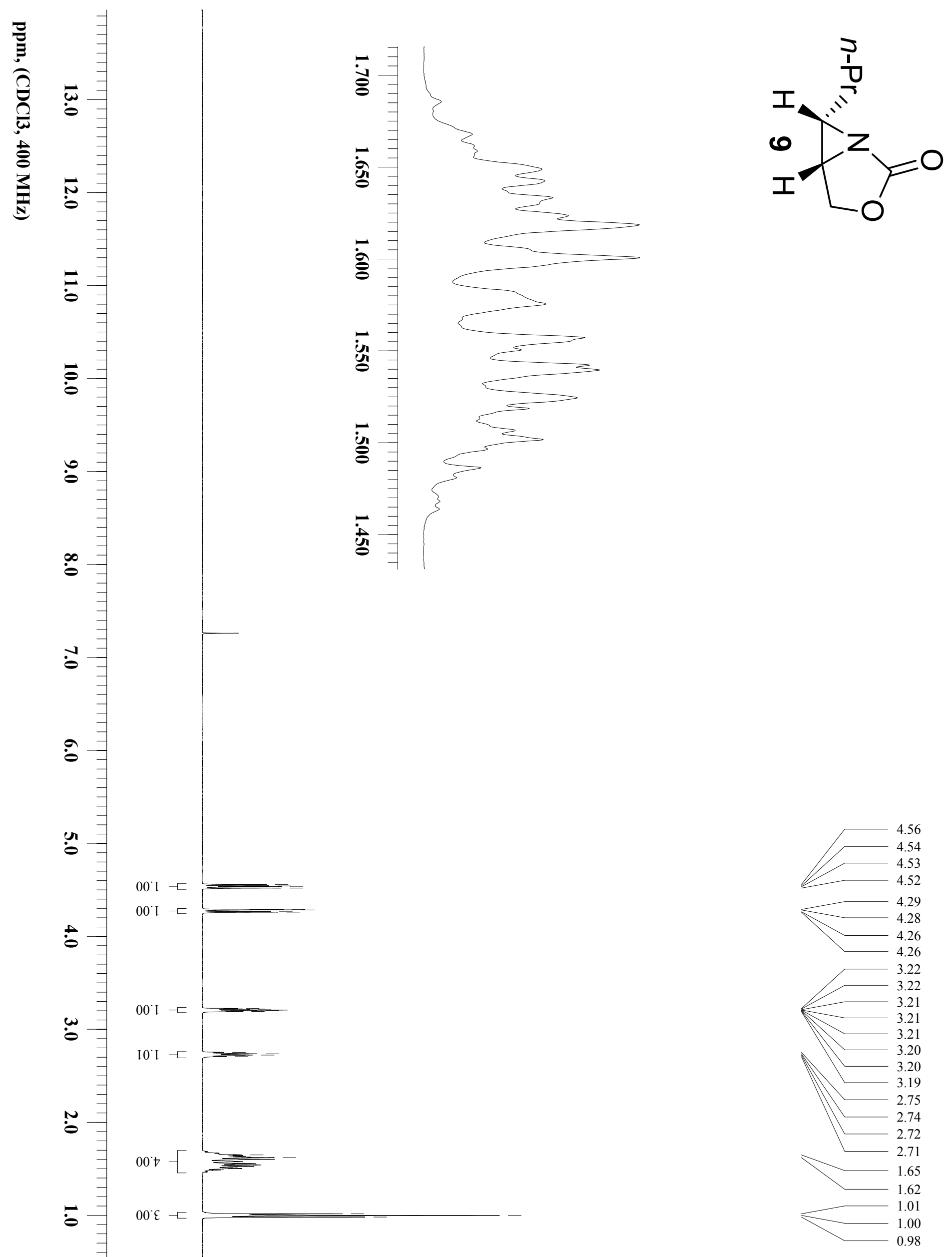


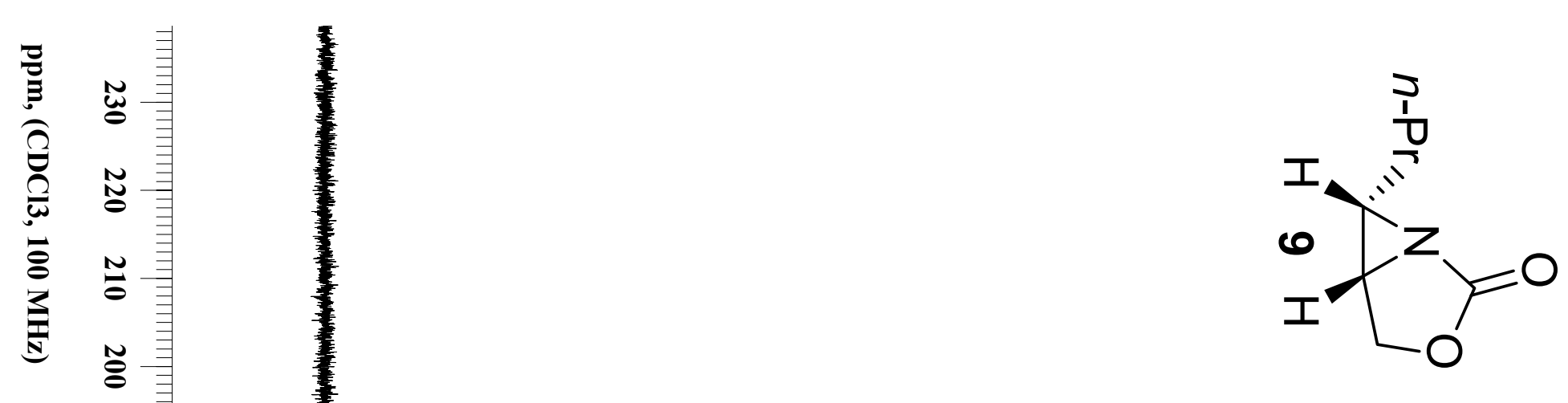

$\overline{8}$

ळ

킁

$\overline{8}$

당

t

$\overline{\mathscr{\sigma}}$

ิㅡㅇ

$\Xi$

\&

๖

$\stackrel{\infty}{\circ}$

니

g

y)

刍

岁

ำ

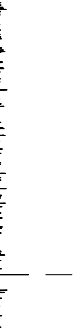



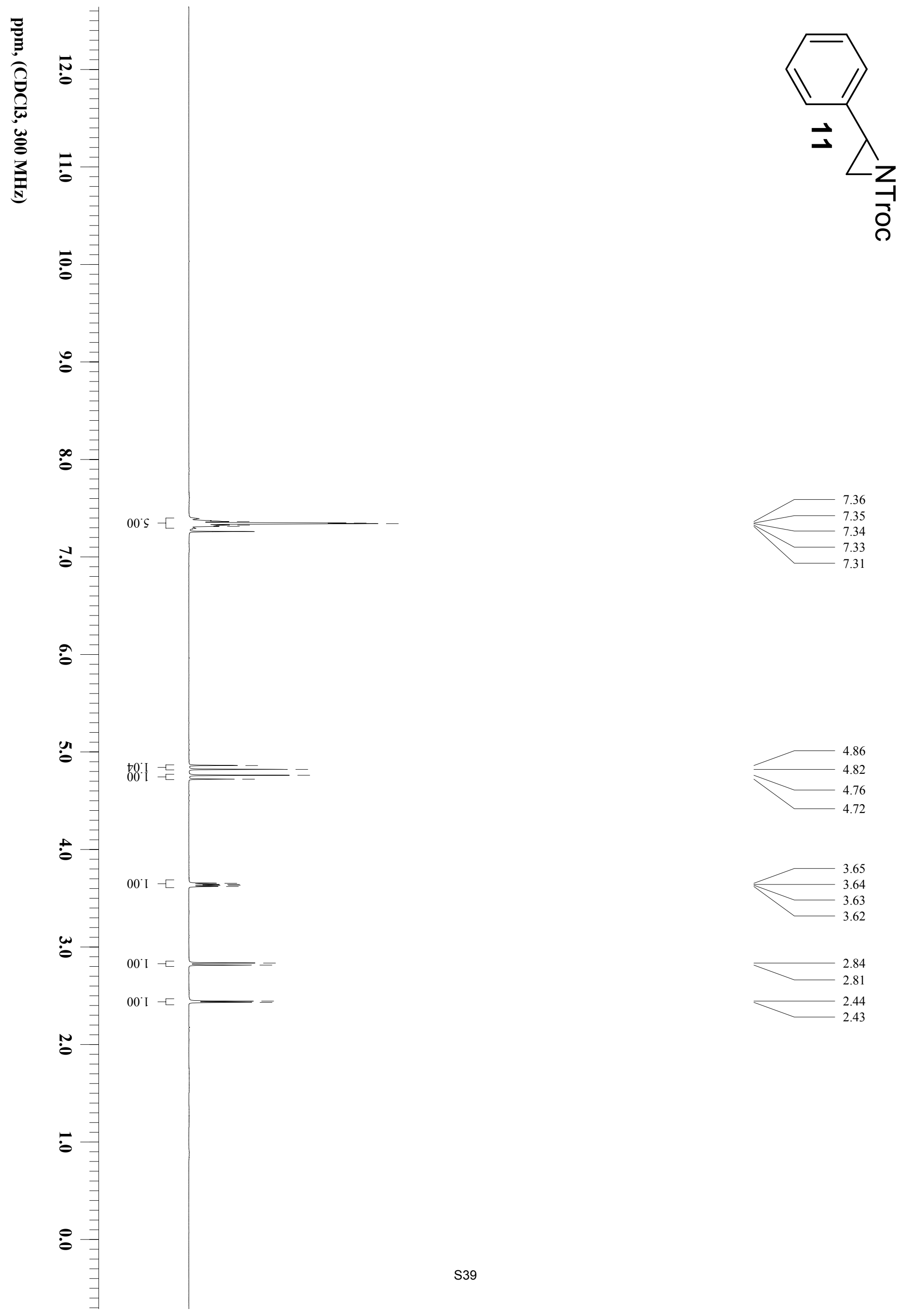


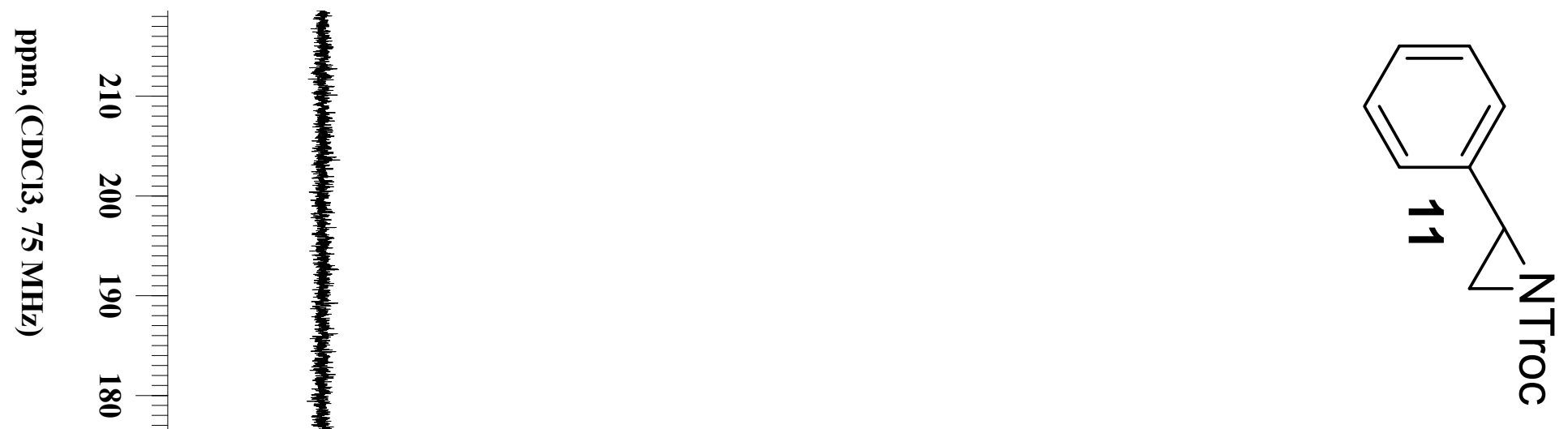

161.31

횽

E

136.11

$\bar{\Xi}$

128.47

128.00

126.14

ฐ

E

$\bar{\Xi}$

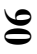

$\stackrel{8}{\circ}$

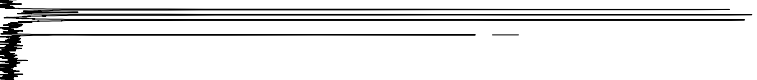

75.27

a

8

낭

E

39.72

35.13 


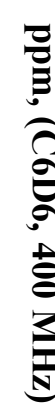

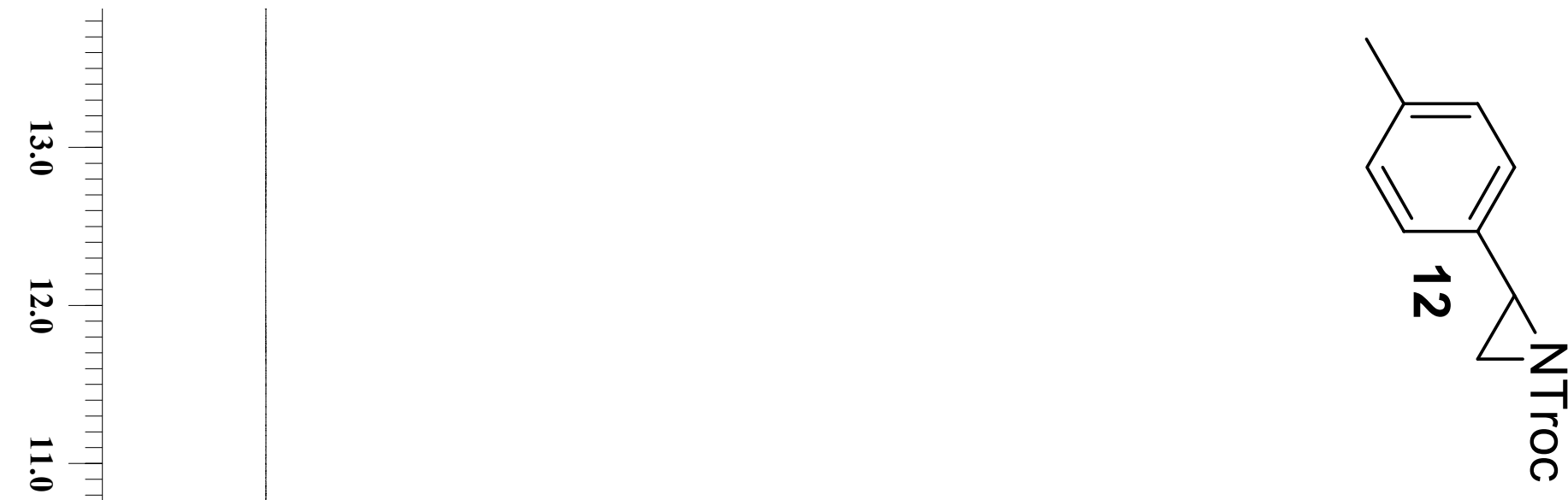




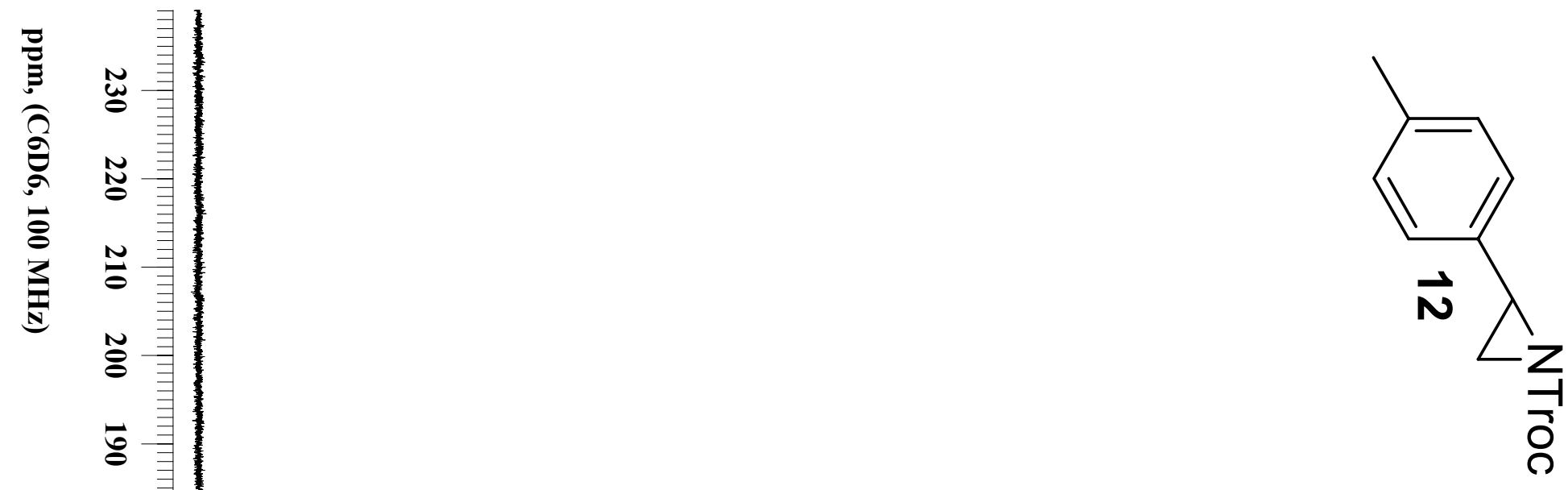

\&

히

홍

161.4

둉

E

๘્વ

ิㅗㅇ

ప

\&

๖

$\stackrel{8}{\circ}$

ㄴ

을

닝

t

40.0

35.2

$\ddot{g}$

옹

21.1 

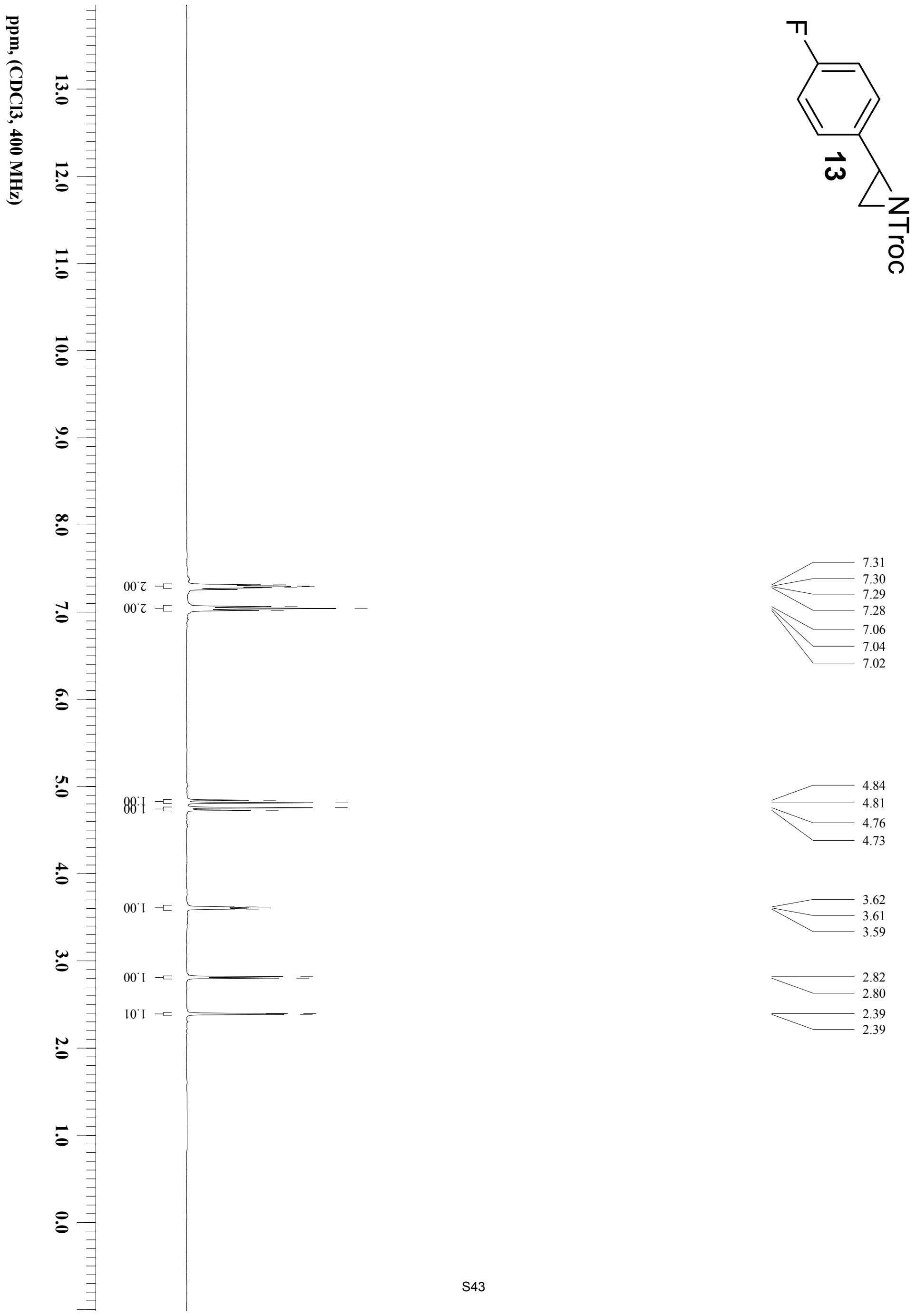
З̊ำ

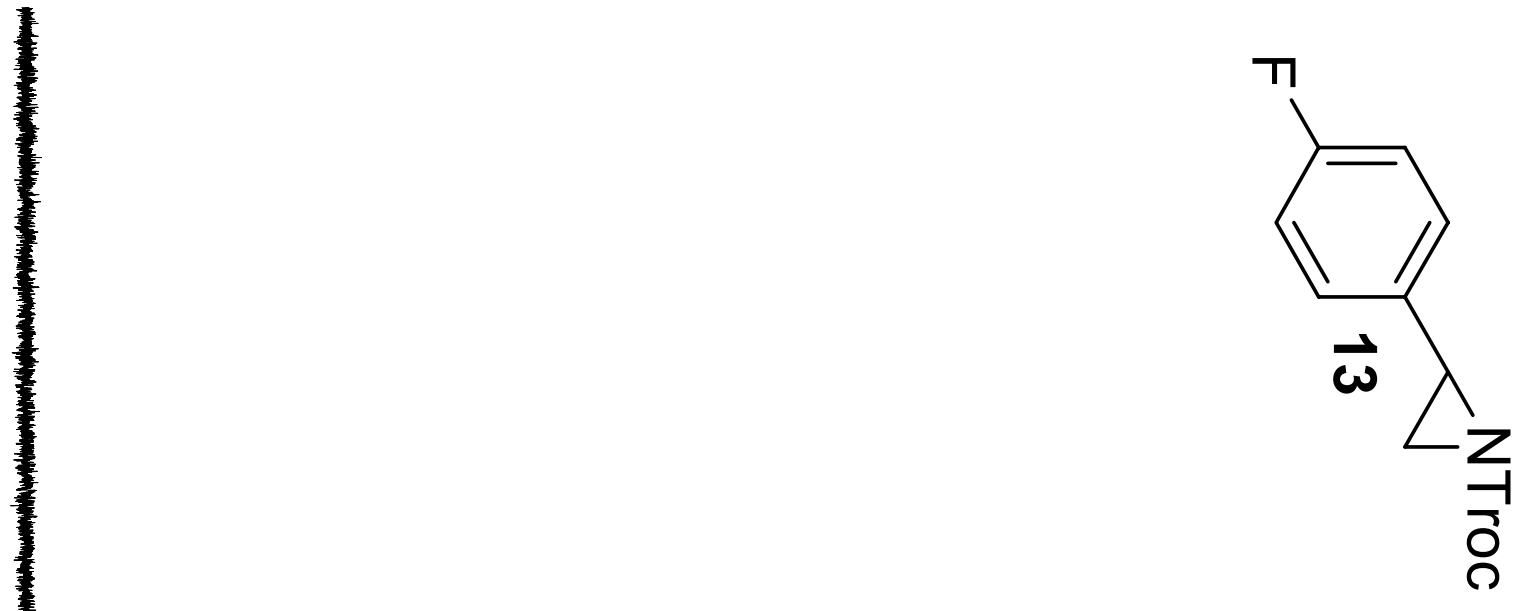

$$
5
$$$$
\text { ริ }
$$

빙

E

g

ธิ

z

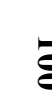

8

$\infty$ 

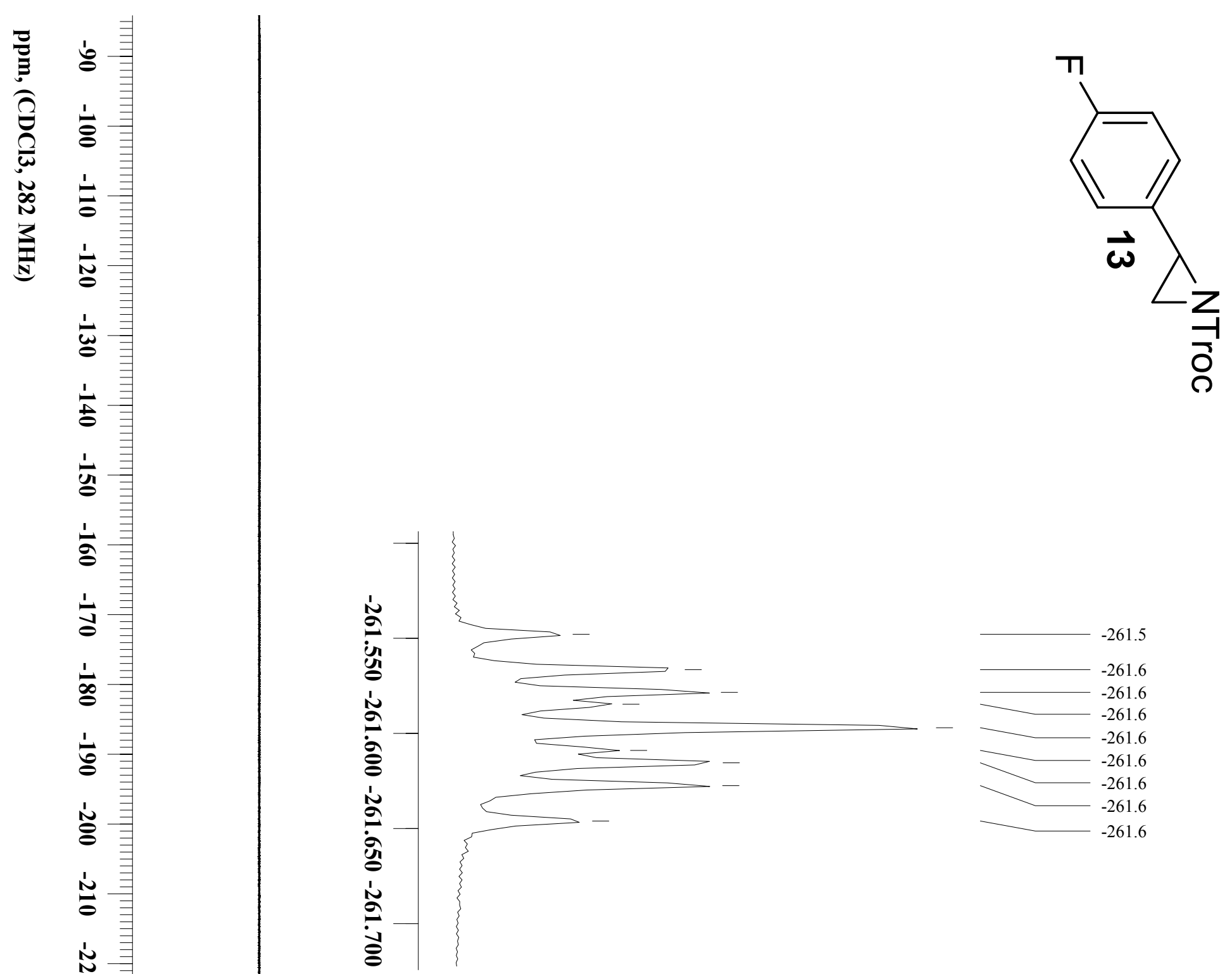

Ñ

$\stackrel{N}{+}$

离

ลั

ปે

$\stackrel{\infty}{\infty}$

ํํㅇ

હ્̊

w

心 

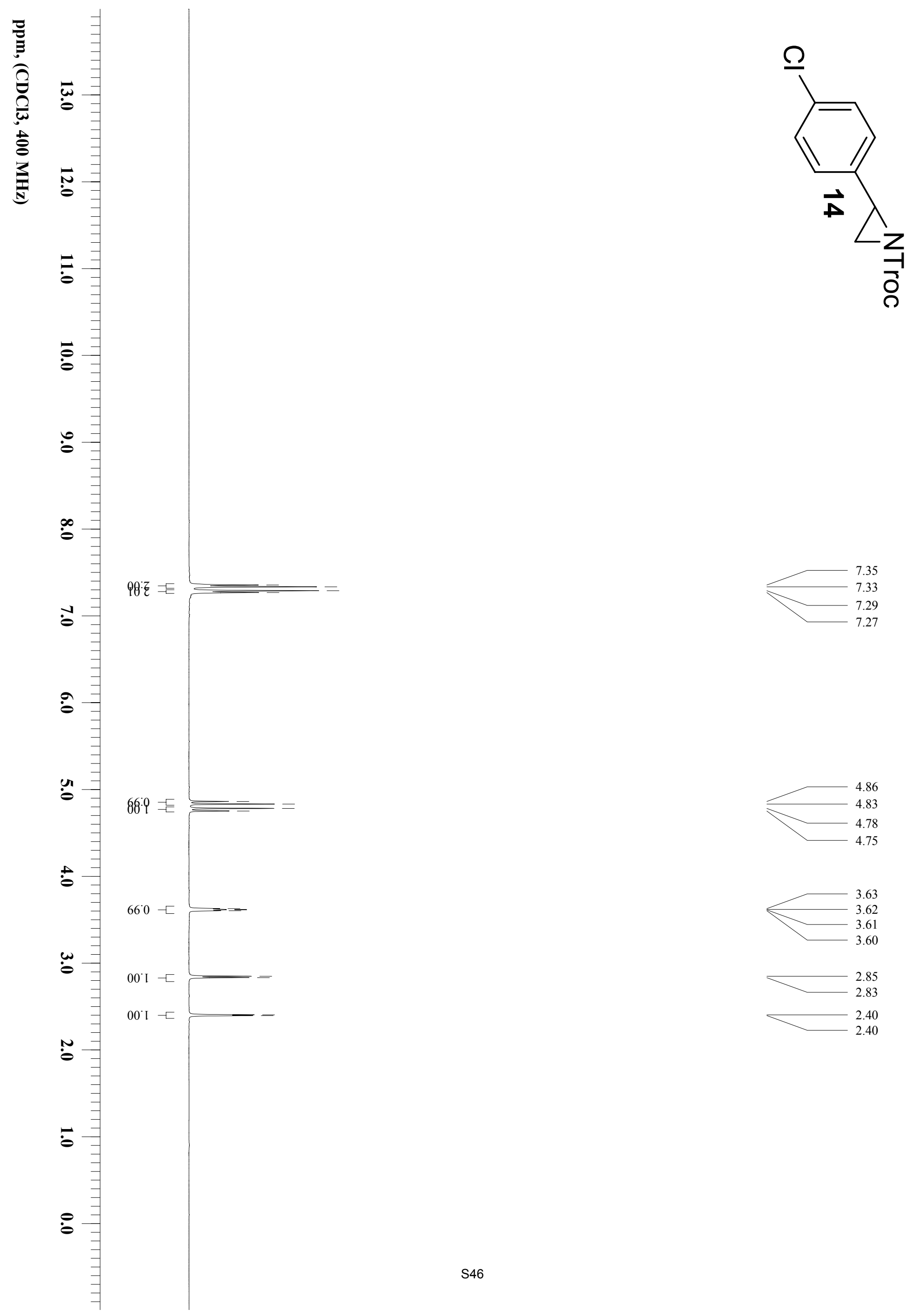

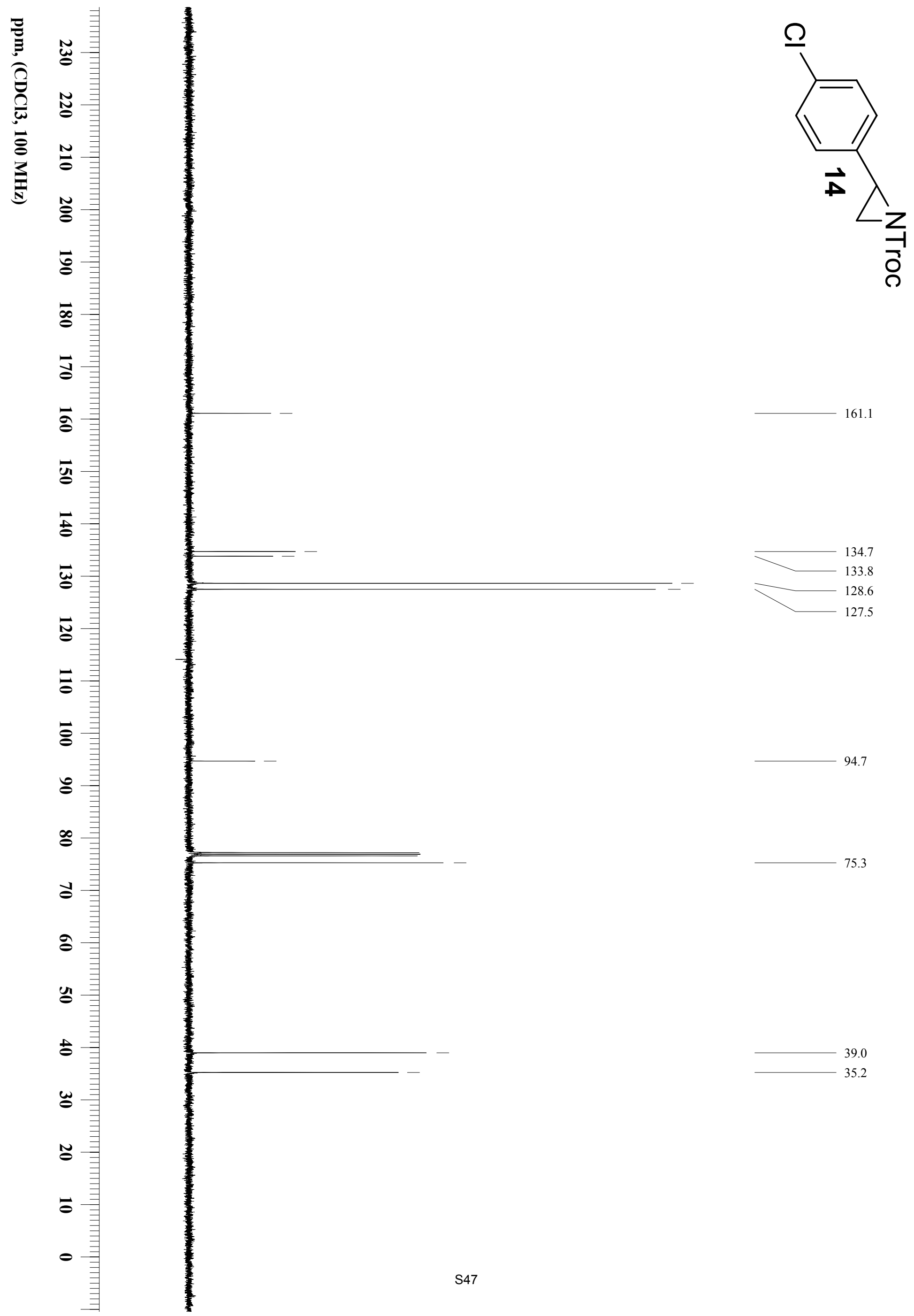

161.1

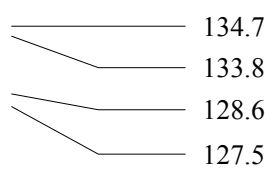

94.7 

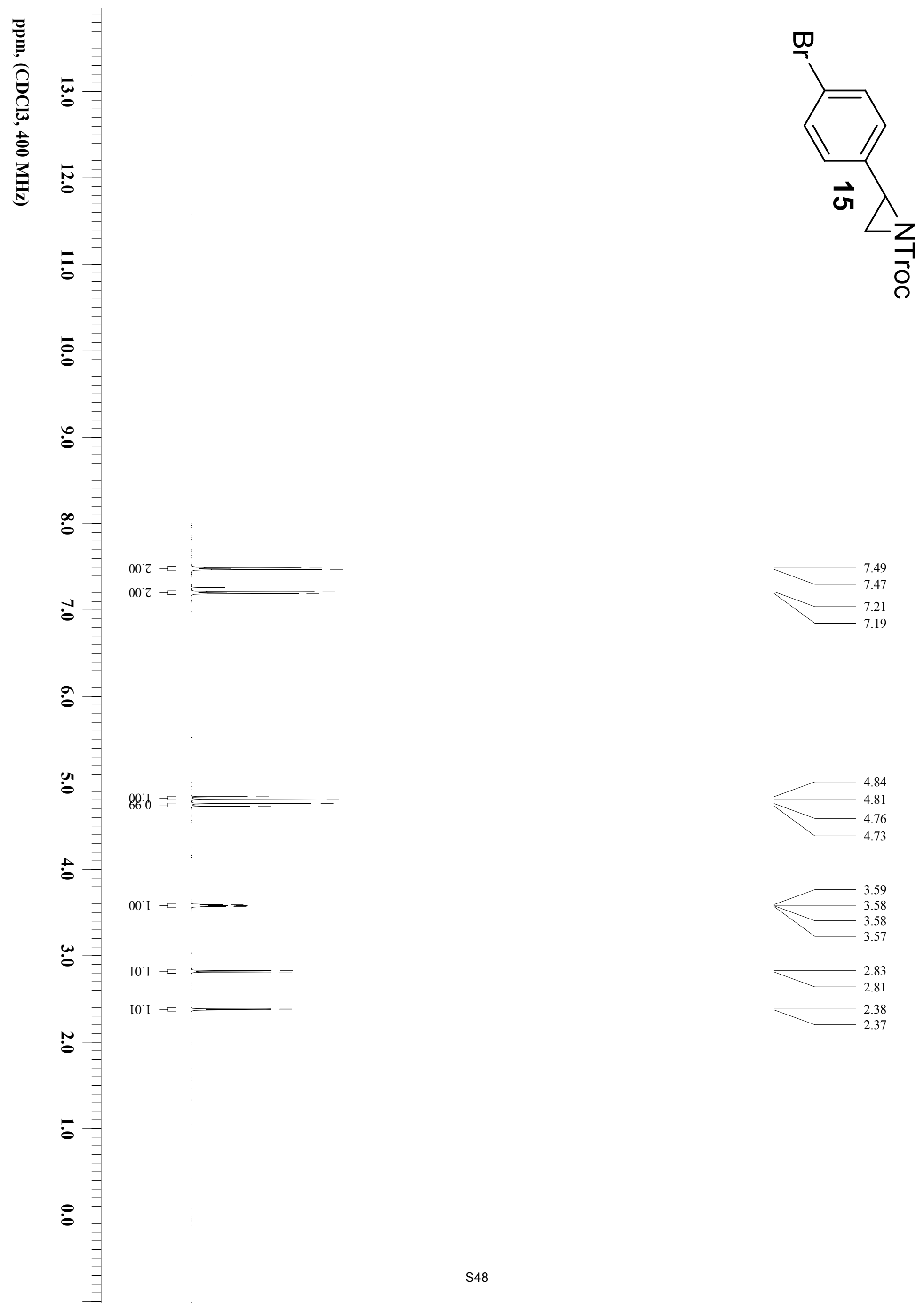


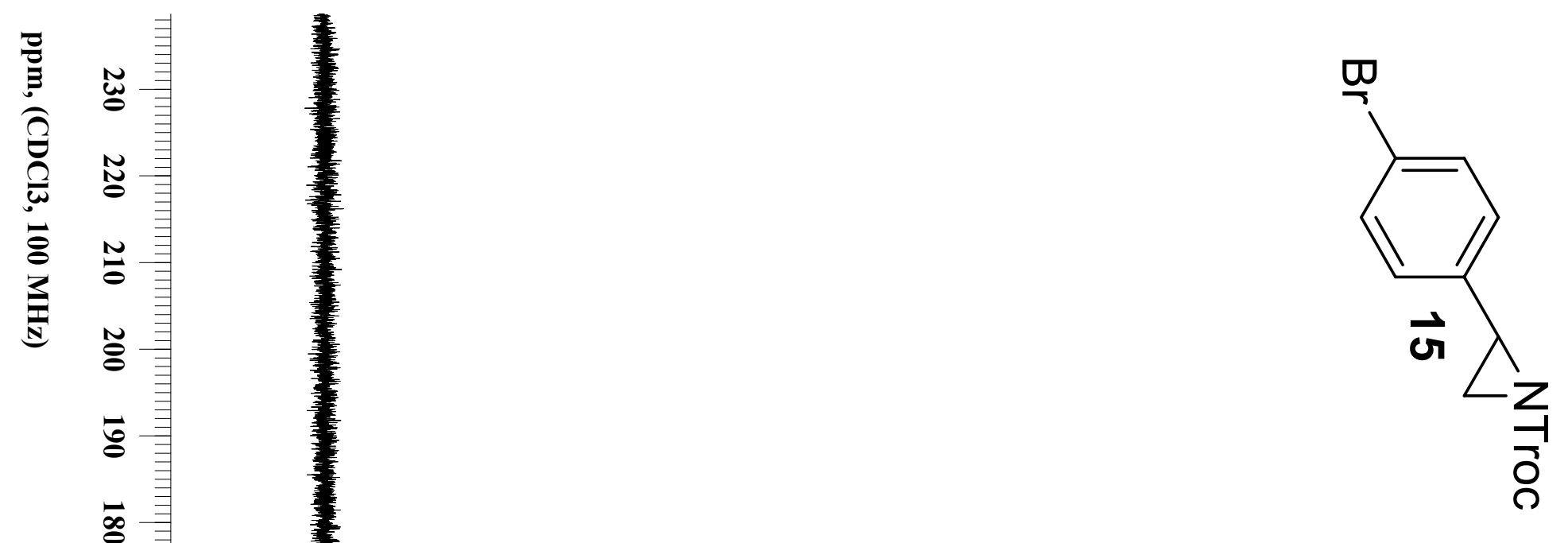

- 161.1

135.3

131.6

ம)

ㅊ.

122.0

E

ธ

8

$\infty$

ฮे

8

용

t

$\ddot{\theta}$

ก

。

- 


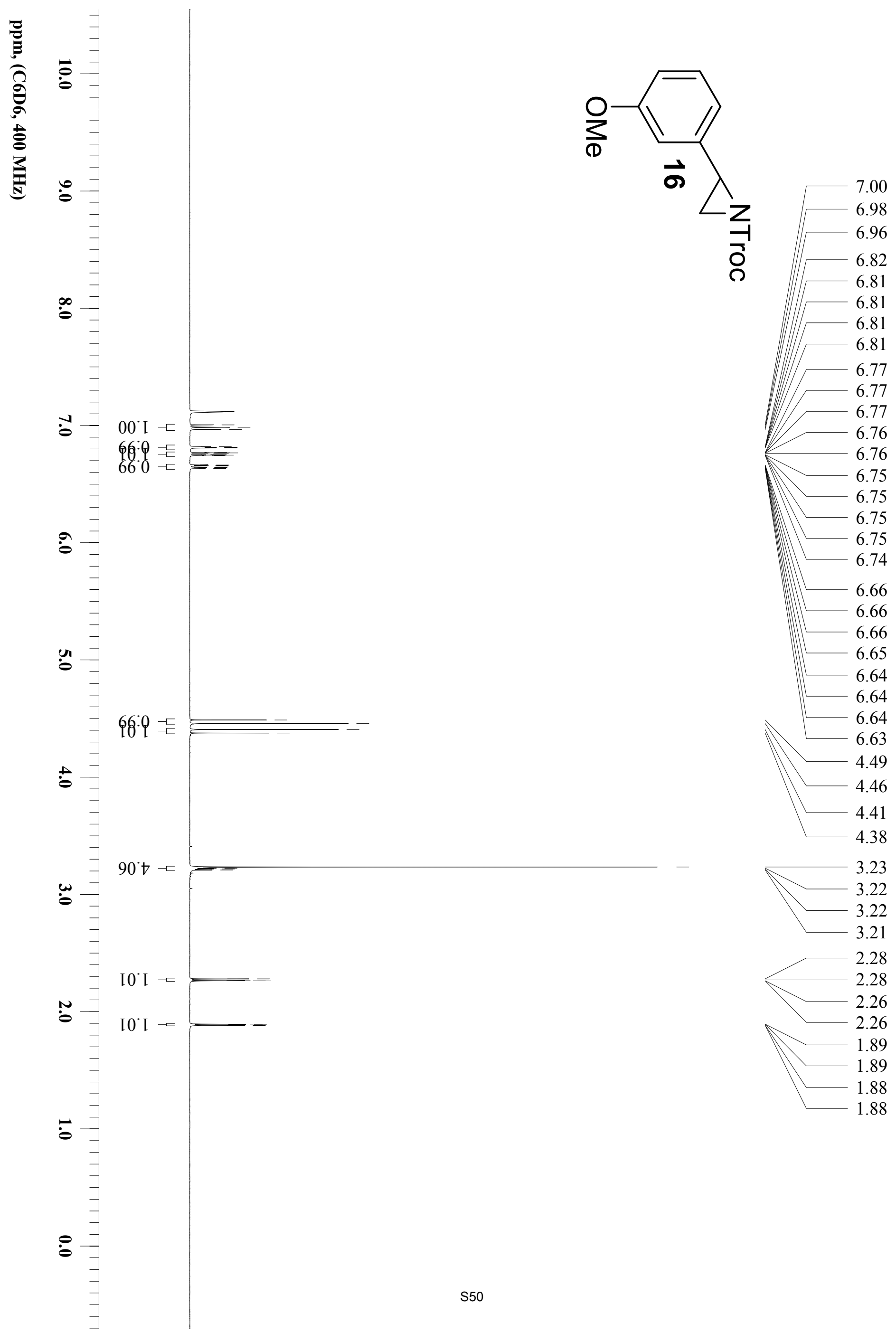



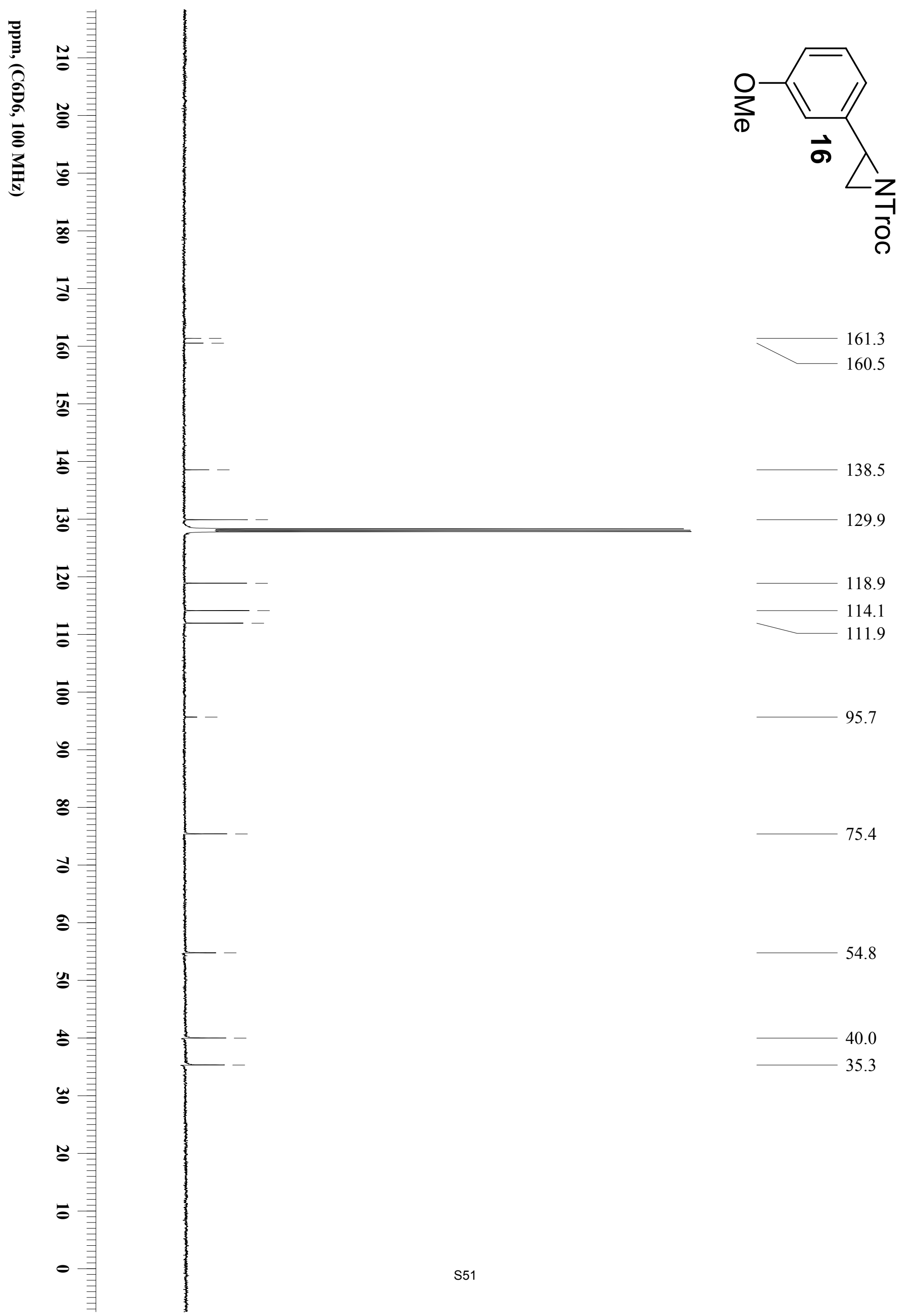

161.3

160.5

138.5

129.9

118.9

114.1

111.9

95.7

75.4

54.8

40.0

35.3 


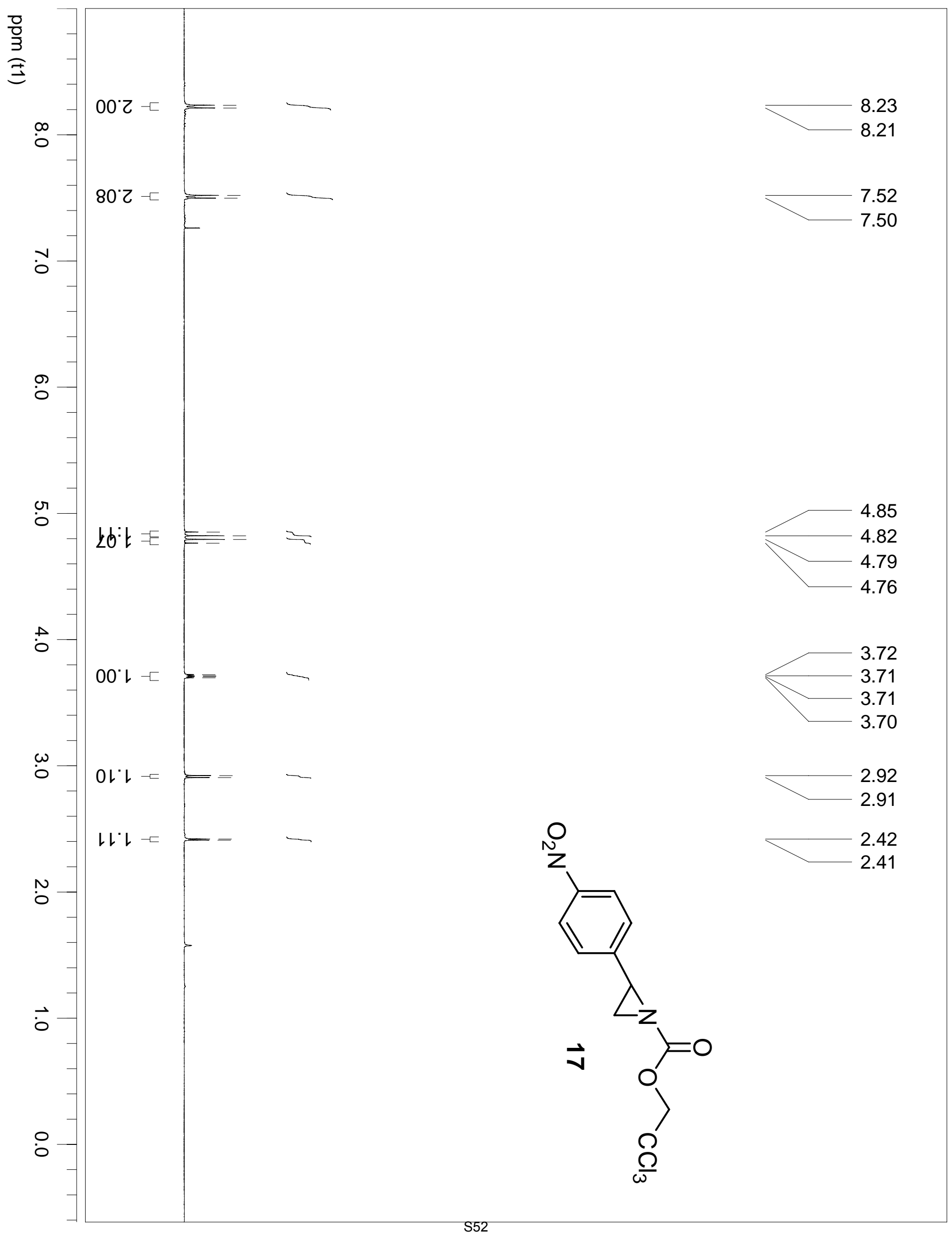




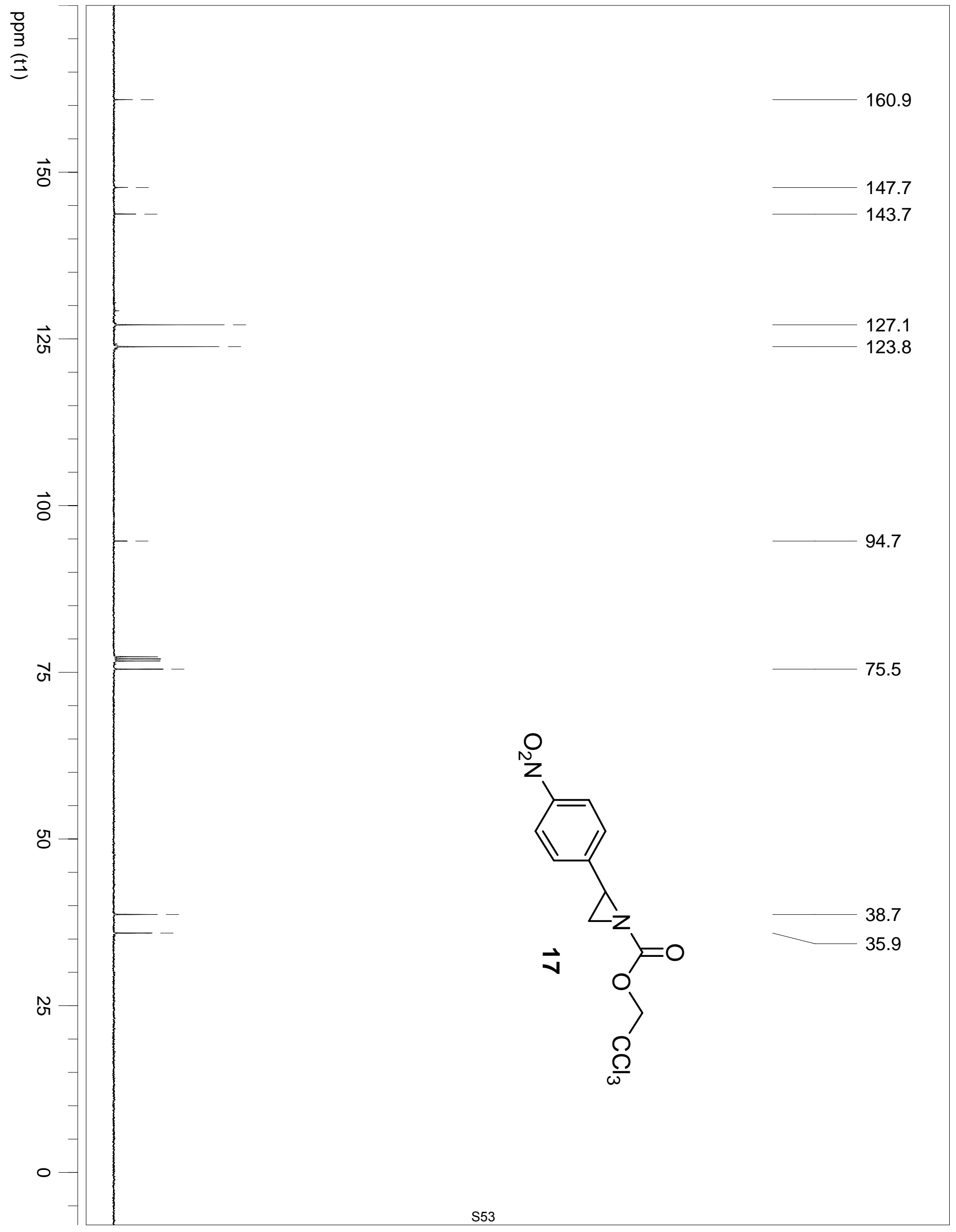




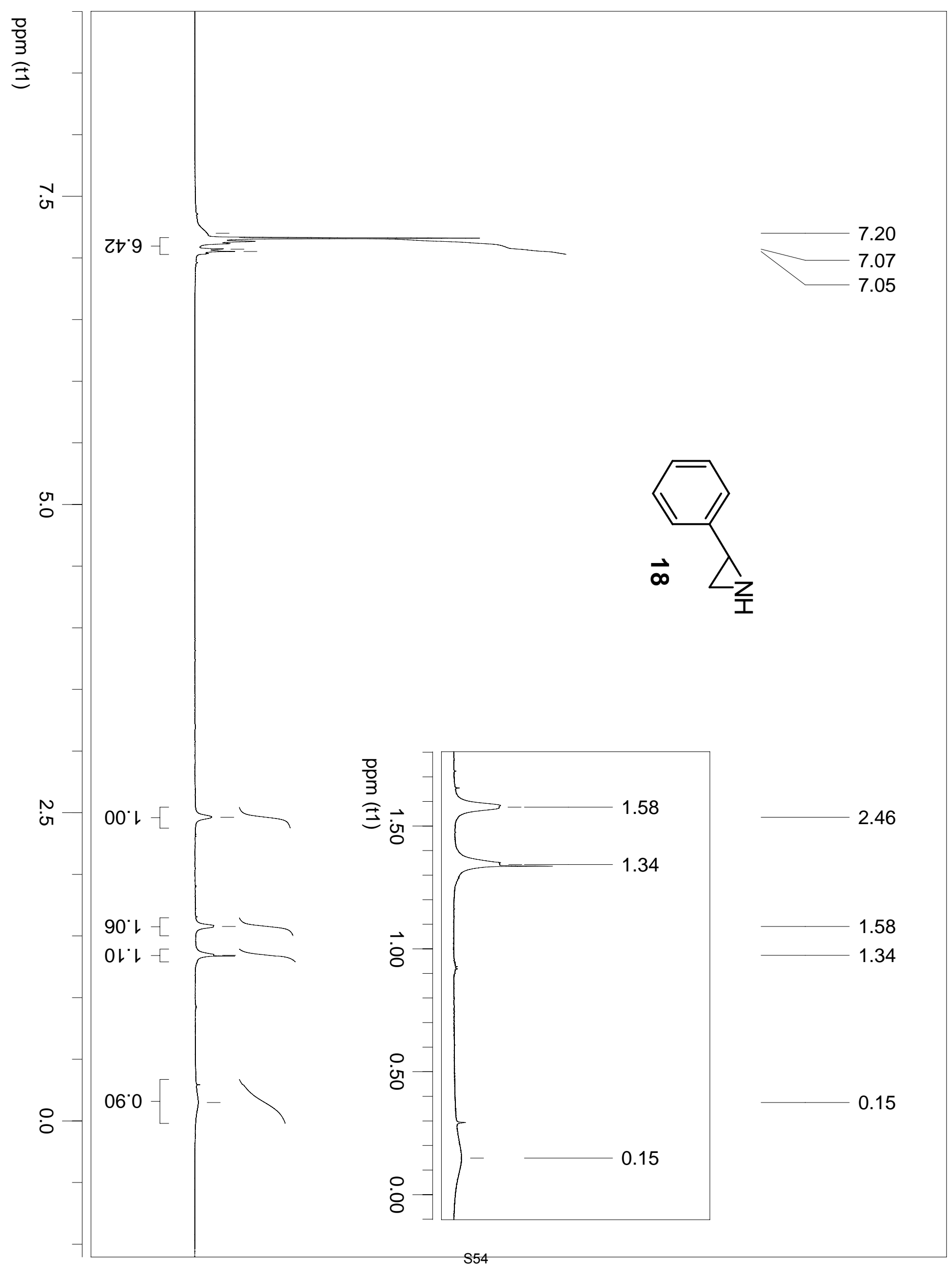




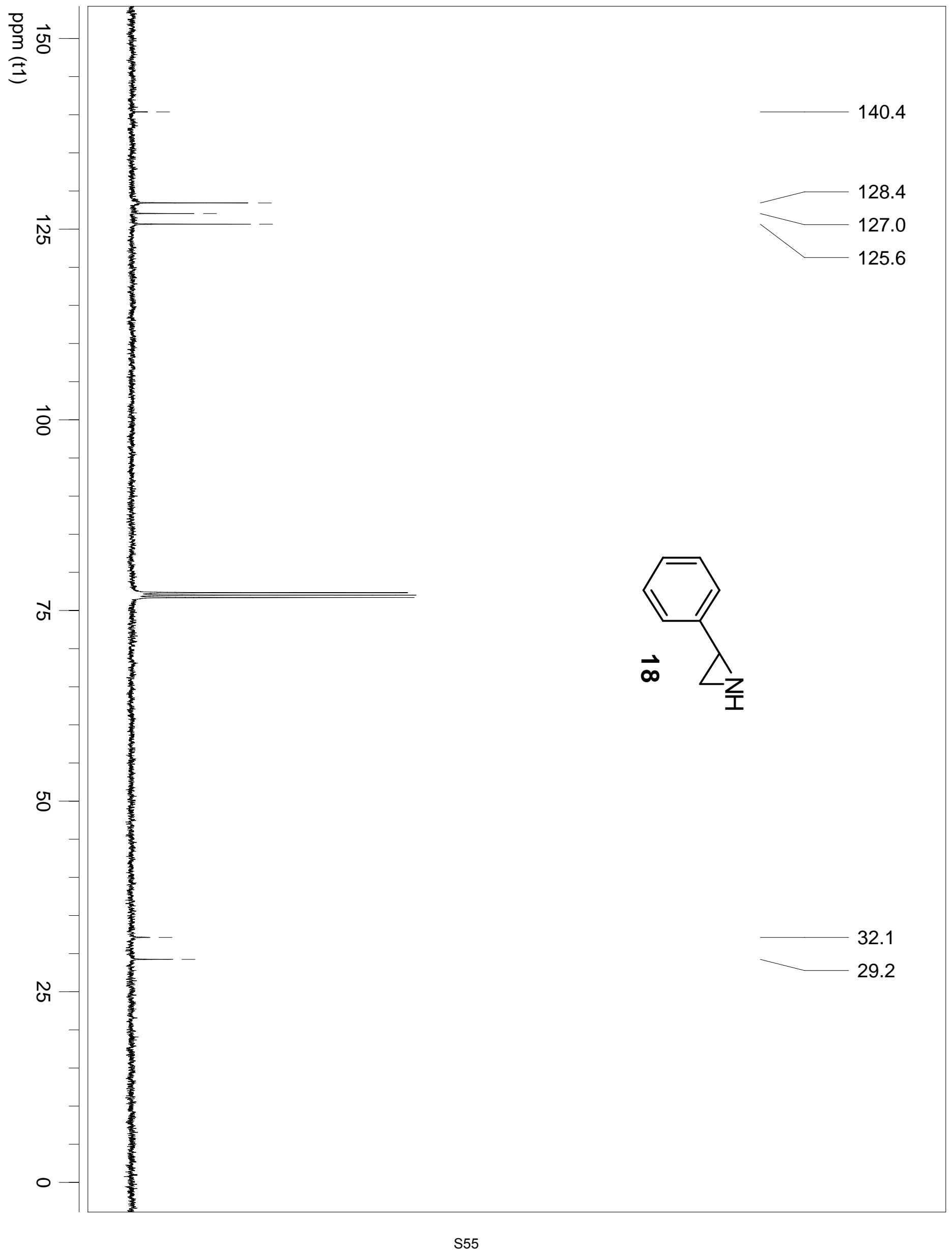




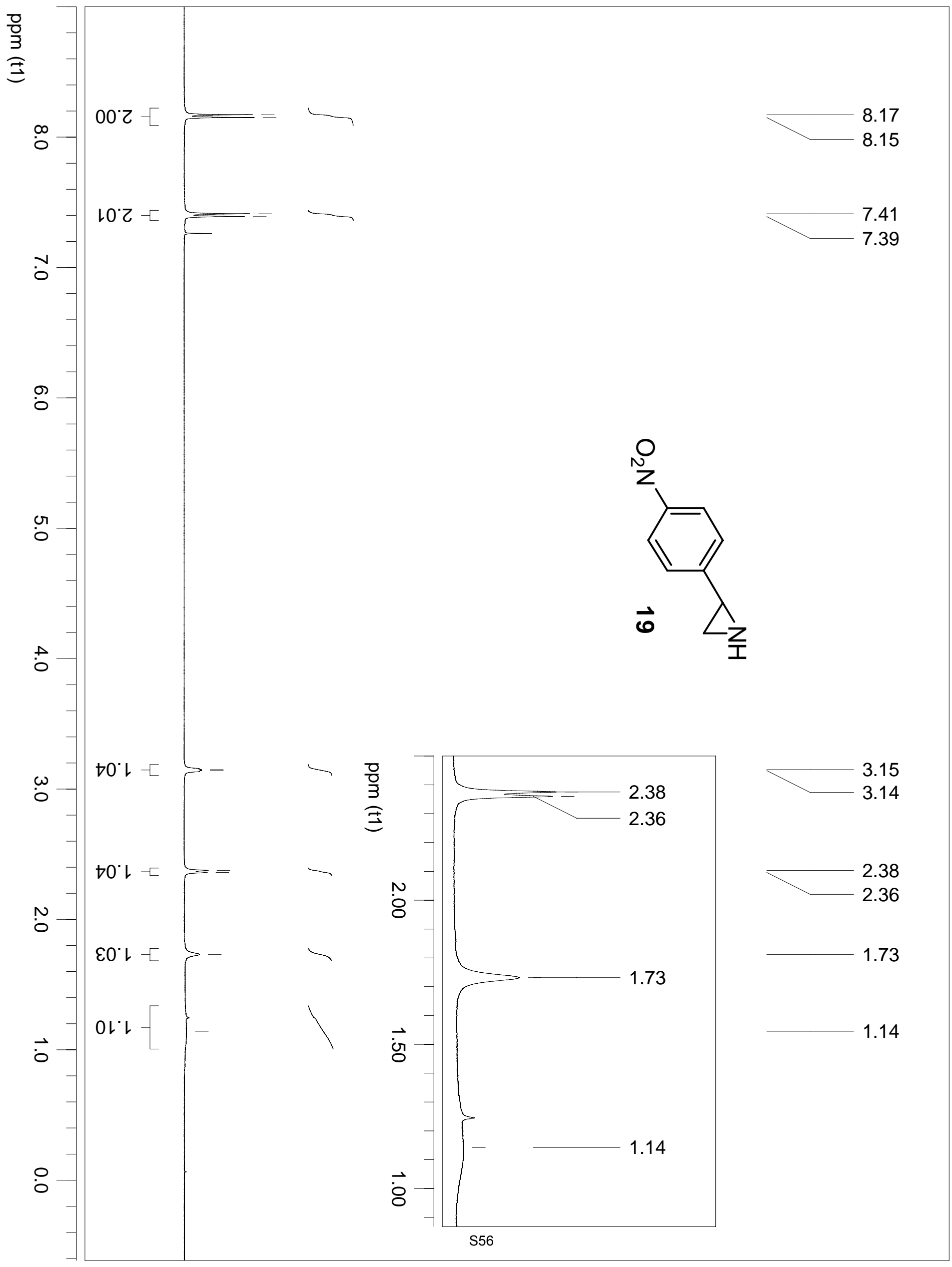




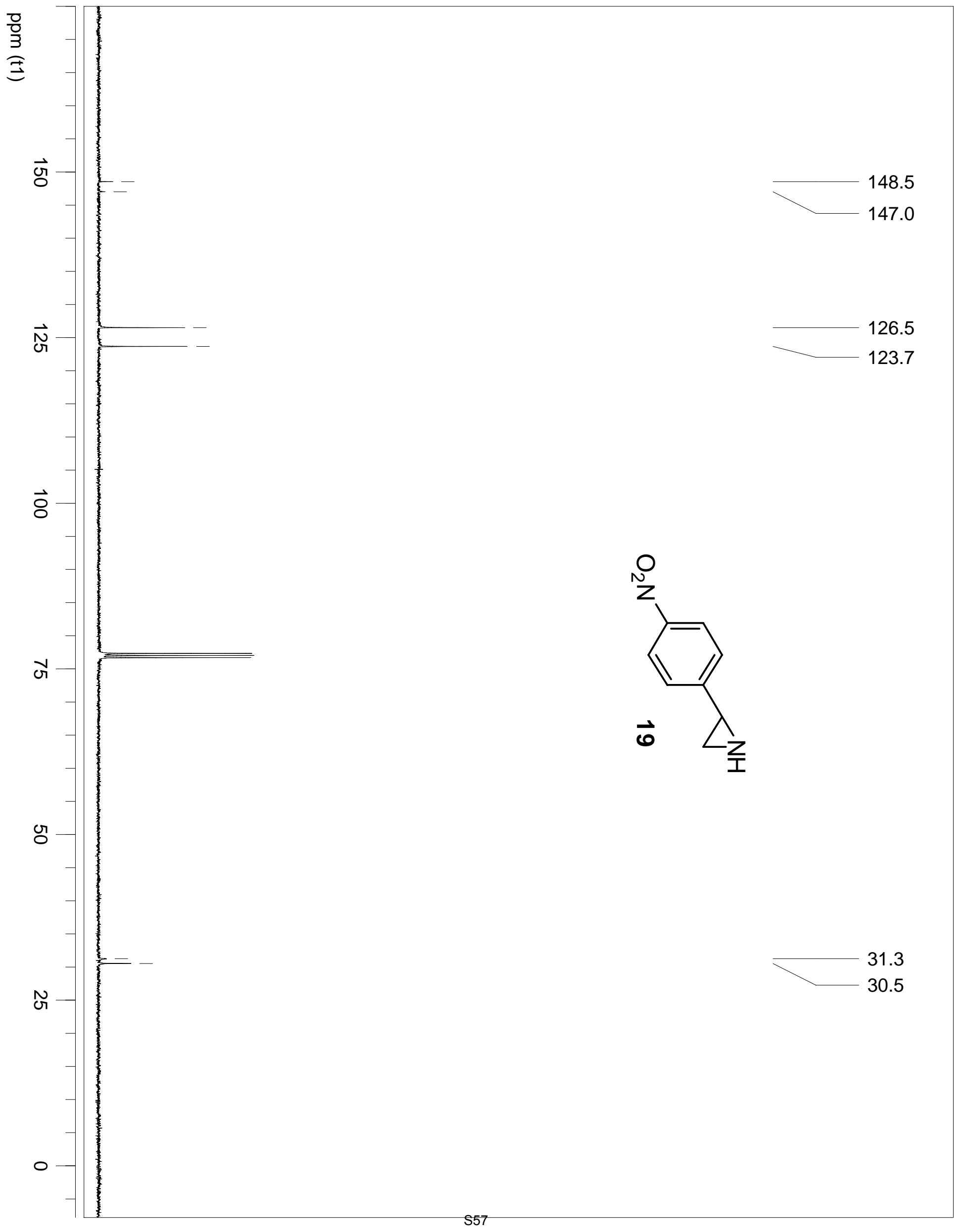

